\title{
Sum Rate Maximization for Cognitive MISO Broadcast Channels: Beamforming Design and Large Systems Analysis
}

\author{
Yuan Yuan He and Subhrakanti Dey, Senior Member, IEEE
}

\begin{abstract}
This paper considers the ergodic weighted sum rate maximization (WSRMax) problem for an underlay cognitive radio multiple input single output (MISO) broadcast channel. In this setting, a secondary network, consisting of a base-station with $M$ transmit antennas and $K$ single-antenna secondary users (SUs), is allowed to share the same spectrum with a primary user (PU), under an average total transmit power (ATTP) constraint and an average interference power (AIP) constraint at the PU receiver. We show that the ATTP constraint always remains active, and as the maximum ATTP $P_{a v} \rightarrow \infty$, the ergodic WSR approaches infinity similar to conventional nonCR networks. We propose a novel low-complexity suboptimal beamforming scheme termed "Partially-Projected \& Regularized Zero-Forcing Beamforming" (PP-RZFBF) with a close-form beamformer, by combining the regularized zero-forcing (RZF) with the channel projection idea, to achieve a tradeoff between maximizing secondary throughput and suppressing secondary multiuser interference as well as the interference on PU. In order to analyze and optimize the performance of this scheme, we employ the large system analysis technique, in the limit as $M$ and $K$ approach infinity with a fixed ratio $r=\frac{K}{M}$. This allows us to derive deterministic limiting approximations for the PPRZFBF problem which enables us to determine asymptotically optimal beamformers for PP-RZFBF. In the large system limit, for the PP-RZFBF scheme, we also find that as $P_{a v} \rightarrow \infty$, the interference on PU caused by the secondary transmission is asymptotically removed. A special suboptimal beamforming scheme called "CZFBF" is also considered, which involves zero forcing all the interference, including the secondary multiuser interference as well as the interference imposed on PU. Various interesting comparisons between PP-RZFBF and CZFBF are provided. Numerical simulations illustrate that the asymptotically optimal beamformers for the PP-RZFBF scheme provide an excellent performance even for finite-sized systems.
\end{abstract}

Index Terms-Underlay cognitive radio, secondary multipleinput single-output broadcast channel, weighted sum rate maximization, beamforming design, large system analysis.

\section{INTRODUCTION}

$\mathbf{C}$ OGNITIVE radio (CR) has been promoted as a promising approach for dramatically increasing the efficiency of spectrum utilization, it involves allowing unlicensed/secondary users (SUs) to access the spectrum originally licensed to

Manuscript received June 19, 2013; revised October 19 and December 20, 2013; accepted January 31, 2014. The associate editor coordinating the review of this paper and approving it for publication was S. Jin.

This research was supported by the ARC Discovery grant DP0984862.

Y. Y. He is with the Electrical and Computer Systems Engineering, Monash University, Australia (e-mail: katrina.he@monash.edu).

S. Dey is with the Department of Signals \& Systems, Uppsala University, Uppsala, Sweden (e-mail: Subhra.Dey@signal.uu.se).

Digital Object Identifier 10.1109/TWC.2014.031314.131097 primary users (PUs), under various operational constraints. Effectively, three categories of CR network paradigms have been proposed: interweave, overlay, and underlay [1]. This paper will focus on underlay systems, where the SUs can transmit simultaneously with PUs, as long as the resulting interference on PUs does not exceed a tolerable limit. Therefore the transmission strategies of SUs should be designed properly so as to achieve the best trade-off between maximizing the secondary throughput and minimizing the interference imposed on PUs.

The well-studied multi-antenna (MIMO) technology has been increasingly used in the secondary network to significantly boost the secondary throughput. A CR point-topoint MIMO network throughput maximization problem was optimally solved in [2], which first introduced the 'channel projection' idea (projecting secondary channels into certain null space of secondary-to-primary interference channels) to design suboptimal transmission schemes. It also showed that beamforming $(\mathrm{BF})$ is the optimal transmit strategy for the corresponding MISO case. In [3], the authors considered the sum-rate maximization (SRMax) problem in a CR SIMO multiple access channel (MAC). In [4], the authors investigated the weighted SRMax (WSRMax) problem for CR Multi-SUs MIMO broadcast channel (BC) with a dirty paper coding (DPC) precoder (motivated by the fact that DPC is the optimal capacity achieving scheme for non-CR MIMO BC [5]), which is shown to be a nonconvex problem but can be transformed into an equivalent convex CR MIMO MAC problem by applying the notion of BC-MAC duality. Throughput maximization problems with linear BF schemes for CR MISO interference channel (IFC) networks were studied in [6] and [7], where in [6], the authors proposed distributed BF and rate allocation for maximizing the smallest weighted rate. See also [7], where the authors used an iterative algorithm to find a locally optimum for the non-convex WSRMax problem. In the case of CR MIMO ad hoc networks, the authors of [8] derived a semidistributed algorithm and an alternative centralized algorithm for obtaining a locally optimal linear precoder for the nonconvex WSRMax problem. A CR multi-antenna two-way relay network was considered in [9], which proposed optimal relay BF structure and suboptimal relay BF schemes with a subspace projection idea similar to [2]. In [10], the authors also provided a overview of various CR MIMO networks.

In this paper, we address an ergodic secondary WSRMax problem for an underlay CR multi-SUs MISO BC (CR-MISOBC) network, as shown in Fig.1, where a secondary multi- 
user MISO downlink is allowed to share the same spectrum with an existing single-antenna PU, subject to an average total transmit power (ATTP) constraint at the secondary basestation (CR-BS) and an average interference power (AIP) constraint at the PU to limit the interference caused by the secondary transmission. The system setting is similar to [4], but unlike [4], we consider a linear precoding transmit strategy instead of nonlinear DPC. This is motivated by the fact that despite the optimality of DPC, it is too complex to be implemented in practical systems. The suboptimal but lowcomplexity linear precoding technique provides a good tradeoff between performance and complexity, and consequently much more attractive in practice. Similar to $\mathrm{CR}$ point-to-point MISO case [2], we also find that BF is the optimal secondary transmit strategy for our CR-MISO-BC. Although the CRMISO-BC WSRMax problem is non-convex and NP-hard, a few existing algorithms for solving WSRMax problem of traditional non-CR networks [11][12][13][14][15][16] and CR networks [8][7], can be easily applied to find a globally/locally optimal downlink BF. As an example, we design a locally optimal BF algorithm named "Beamforming via Lagrangian dual problem with zero-gradient iterative method" (BF-LDPZGIM), based on a ZGIM algorithm proposed by [15][7]. Due to space constraints, we do not include its details but they can be found in Section III-A of a longer version of this paper [17]. Almost all of these algorithms generally have high computational complexity and can only obtain the optimal BF numerically with no explicit form, thus hindering any further theoretical analysis. Motivated by this, in this paper, we will focus on deriving low-complexity albeit suboptimal closedform beamformers for the CR-MISO-BC secondary WSRMax problem. Our main contributions are summarized as follows:

- In our CR-MISO-BC WSRMax problem, we prove that under all circumstances, the ATTP constraint always remains active, and as the maximum ATTP $P_{a v} \rightarrow \infty$, the ergodic WSR approaches infinity, and the optimal transmit strategy of CR-MISO-BS system is BF. These properties are in contrast with prior research on the CR network with single-antenna transmitters (such as [18][19][3]), where with fixed $Q_{a v}$ (maximum AIP), in the high $P_{a v}$ regime, only the AIP constraint is satisfied with equality and the achievable rate remains upper bounded.

- Motivated by difficulties imposed by the non-convexity of the associated optimization problem, we derive a low-complexity albeit suboptimal strategy (called 'PPRZFBF') with a closed-form beamformer, by combining the regularized zero-forcing (RZF) [20] (well studied in traditional non-CR networks to control multiuser interference) with the 'channel projection' idea proposed in [2][10][9] (to control the secondary interference on PU), in order to achieve a tradeoff between maximizing secondary throughput and suppressing secondary multiuser interference as well as the interference on PU.

- Designing the PP-RZFBF beamformer involves a twodimensional brute-force search for finding two crucial parameters: the optimal regularization parameter $\alpha$ (to control multiuser interference) and the projection control

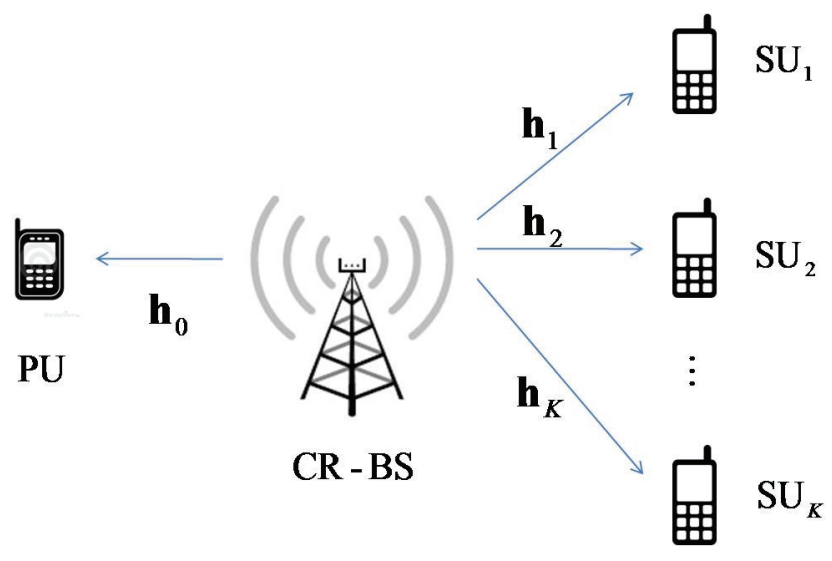

Fig. 1. CR-MISO-BC system model.

parameter $\beta$ (to control the secondary interference on $\mathrm{PU})$, which is obviously computationally rather prohibitive. This motivates us to derive deterministic large system approximations (inspired by [21][22][23][25] which consider non-CR networks and have the regularization parameter $\alpha$ only) for the PP-RZFBF scheme in the large system limit (as $M$ and $K$ tend to infinity with a fixed ratio $r=\frac{K}{M}$ ), which enables us to derive the asymptotically optimal value of $\alpha$ and the range of $\beta$ for the PP-RZFBF scheme. This allows us to apply the resulting values of $\alpha$ and $\beta$ into our proposed PP-RZFBF scheme to obtain a low-complexity suboptimal beamformer for finite-sized practical CR-MISO-BC systems. We also find that in the large system limit, as $P_{a v} \rightarrow \infty$, for the PP-RZFBF scheme, the interference on PU caused by the secondary transmission is asymptotically removed, i.e, the AIP constraint becomes asymptotically inactive. ${ }^{1}$

- We also propose a special suboptimal beamforming scheme 'CZFBF' by zero forcing all the interference (including the secondary multiuser interference as well as the interference imposed on PU due to the secondary transmission). Various interesting comparisons between PP-RZFBF and CZFBF are provided.

Finally note that a preliminary version of this work with some partial results has been presented in a conference paper [28]. The current journal version provides a significantly detailed and extended version with rigorous mathematical analysis and a number of novel theoretical results in the large systems analysis of CR-MISO-BC systems.

\section{System Model and Problem Formulation}

We consider an underlay cognitive radio MISO broadcast channel (CR-MISO-BC), as illustrated in Fig.1, where a secondary base station (CR-BS) equipped with $M \geq 2$ transmit antennas communicates with $K$ single-antenna secondary users (SUs), in the presence of a single-antenna primary user (PU). Regardless of the on/off status of the PU, the secondary network is allowed to share the same spectrum with the PU,

\footnotetext{
${ }^{1}$ See also [24] for similar results for the non-CR scenario in massive MIMO networks even with hardware impairments at both the base station and user equipments.
} 
as long as the degradation of the received signal quality of PU caused by the interference due to the secondary transmission is below an acceptable level. Let $\mathbf{h}_{i} \in \mathbb{C}^{M \times 1}, i=1, \ldots, K$ denote the channel vector from the CR-BS to the $i$-th SU (denoted by $\mathrm{SU}_{i}$ ) and $\mathbf{h}_{0} \in \mathbb{C}^{M \times 1}$ represent the channel vector from the CR-BS to the PU. All channels involved are assumed to be flat-fading, and the elements of each $\mathbf{h}_{j}, \forall j=0,1, \ldots, K$ are assumed to be i.i.d complex Gaussian random variables with zero mean and unit variance. The optimal Gaussian codebook is used by the transmitter at CRBS. Let ${ }^{\mathcal{H}}$ denote the Hermitian transpose and $\mathbf{x}_{i} \in \mathbb{C}^{M \times 1}$ be the desired signal vector to be transmitted to the $\mathrm{SU}_{i}$, then the received signal at the $\mathrm{SU}_{i}$, is given as,

$$
y_{i}=\mathbf{h}_{i}^{\mathcal{H}} \mathbf{x}_{i}+\sum_{j=1, j \neq i}^{K} \mathbf{h}_{i}^{\mathcal{H}} \mathbf{x}_{j}+n_{i}, \quad i=1, \ldots, K,
$$

where $n_{i}$ is the additive white Gaussian noise at the receiver $\mathrm{SU}_{i}$ with $n_{i} \sim \mathcal{C N}(0,1) .{ }^{2}$ Let $\mathbf{S}_{i} \succeq 0 \in \mathbb{C}^{M \times M}$ indicate the transmit signal covariance matrix for $\mathrm{SU}_{i}, \forall i=1, \ldots, K$. Then the average total transmit power (ATTP) constraint is given as $E\left[\sum_{i=1}^{K} \operatorname{tr}\left\{\mathbf{S}_{i}\right\}\right] \leq P_{a v}\left(P_{a v}\right.$ is the maximum average transmit power), where the expectation is taken over all channel states $\left\{\mathbf{h}_{0}, \mathbf{h}_{1}, \ldots, \mathbf{h}_{K}\right\}$. $^{3}$

Each secondary receiver only decodes its desirable information and treats the remaining interference as noise. Thus given $\mathbf{H} \triangleq\left\{\mathbf{h}_{1}, \ldots, \mathbf{h}_{K}\right\} \in \mathcal{C}^{M \times K}, \mathbf{h}_{0}$ and $\mathbf{S} \triangleq\left\{\mathbf{S}_{1}, \ldots, \mathbf{S}_{K}\right\}$, the signal to interference plus noise ratio (SINR) at each receiver $\mathrm{SU}_{i}$ is defined as,

$$
\operatorname{SINR}_{i}=\frac{\mathbf{h}_{i}^{\mathcal{H}} \mathbf{S}_{i} \mathbf{h}_{i}}{1+\sum_{j=1, j \neq i}^{K} \mathbf{h}_{i}^{\mathcal{H}} \mathbf{S}_{j} \mathbf{h}_{i}}, \quad i=1, \ldots, K .
$$

The ergodic WSR of this CR-MISO-BC system is then given as,

$$
R_{\text {sum }}=\sum_{i=1}^{K} w_{i} E\left[\log \left(1+\operatorname{SINR}_{i}\right)\right],
$$

where $w_{i}$ is the weight for $\mathbf{S U}_{i}$ and the expectation is taken over all CSI, i.e., $\mathbf{H}$ and $\mathbf{h}_{0}$. A common way to protect PU is to impose either an average or a peak interference power (AIP/PIP) constraint at the PU [29] to regulate the interference. We adopt the AIP constraint in this paper, which can be written as, $E\left[\sum_{i=1}^{K} \mathbf{h}_{0}^{\mathcal{H}} \mathbf{S}_{i} \mathbf{h}_{0}\right] \leq Q_{a v}$, with $Q_{a v}$ denoting maximum average interference power tolerated by the PU.

Therefore, with the assumption of perfect CSI at both transmitter and receivers (i.e., CR-BS knows $\mathbf{H}$ and $\mathbf{h}_{0}$, and each user knows its own channel information), the WSRMax problem for CR-MISO-BC, under both an ATTP constraint at CR-BS and an AIP constraint at the PU, can be formulated as,

\footnotetext{
${ }^{2}$ Here, similar to [2][30], we assume the background noise at each SU receivers includes the interference from the primary transmitter, which is nonwhite in general, but can be assumed to be approximately white Gaussian by applying a noise-whitening filter [2] when the PR transmitter also uses a Gaussian codebook

${ }^{3}$ As we will show in (4), the design of optimal transmit signal covariance matrices $\mathbf{S}_{i}, \forall i=1, \ldots, K$, depends on the channel conditions $\left\{\mathbf{h}_{0}, \mathbf{h}_{1}, \ldots, \mathbf{h}_{K}\right\}$. Thus $\left\{\mathbf{S}_{i}\right\}_{i=1}^{K}$ is a function of $\left\{\mathbf{h}_{0}, \mathbf{h}_{1}, \ldots, \mathbf{h}_{K}\right\}$. For simplicity however, we abbreviate $\mathbf{S}_{i}\left(\mathbf{h}_{0}, \mathbf{h}_{1}, \ldots, \mathbf{h}_{K}\right)$ as $\mathbf{S}_{i}$ throughout the paper.
}

$\underset{\mathbf{S}_{i} \succeq 0, \forall i}{\operatorname{maximize}} R_{\text {sum }}=\sum_{i=1}^{K} w_{i} E\left[\log \left(1+\frac{\mathbf{h}_{i}^{\mathcal{H}} \mathbf{S}_{i} \mathbf{h}_{i}}{1+\sum_{j=1, j \neq i}^{K} \mathbf{h}_{i}^{\mathcal{H}} \mathbf{S}_{j} \mathbf{h}_{i}}\right)\right]$
subject to $E\left[\sum_{i=1}^{K} \operatorname{tr}\left\{\mathbf{S}_{i}\right\}\right] \leq P_{a v}, E\left[\sum_{i=1}^{K} \mathbf{h}_{0}^{\mathcal{H}} \mathbf{S}_{i} \mathbf{h}_{0}\right] \leq Q_{a v}$. (4)

Proposition 1: In Problem (4), the maximum ergodic WSR $R_{\text {sum }} \rightarrow \infty$, as $P_{a v} \rightarrow \infty$; The optimal $\mathbf{S}_{i}, \forall i=1, \ldots, K$ for Problem (4) satisfies $\operatorname{Rank}\left(\mathbf{S}_{i}\right) \leq 1$, i.e., the optimal secondary transmit strategy of CR-MISO-BS system is BF; and given any $P_{a v}$ and $Q_{a v}$, the ATTP constraint in Problem (4) is always satisfied with equality.

Proof: See Appendix A.

Remark 1: The above properties of the CR-MISO-BC system in Proposition 1 are different from prior research on the CR network with single-antenna transmitters (such as [18][19][3]), where in the high $P_{a v}$ regime, only the AIP constraint is active and the achievable rate approaches a constant.

Based on Proposition 1, $\mathbf{S}_{i}(\forall i=1, \ldots, K)$ can be expressed in the form of $\mathbf{S}_{i}=\mathbf{q}_{i} \mathbf{q}_{i}^{\mathcal{H}}$, where $\mathbf{q}_{i} \in \mathbb{C}^{M \times 1}$ denotes the transmit beamforming vector. Then, Problem (4) can be simplified as,

$$
\begin{aligned}
& \underset{\mathbf{q}_{i}, \forall i=1, \ldots, K}{\operatorname{maximize}} R_{\text {sum }}=\sum_{i=1}^{K} w_{i} E\left[\log \left(1+\frac{\left|\mathbf{h}_{i}^{\mathcal{H}} \mathbf{q}_{i}\right|^{2}}{1+\sum_{j=1, j \neq i}^{K}\left|\mathbf{h}_{i}^{\mathcal{H}} \mathbf{q}_{j}\right|^{2}}\right)\right] \\
& \text { subject to } E\left[\sum_{i=1}^{K}\left|\mathbf{q}_{i}\right|^{2}\right] \leq P_{a v}, \quad E\left[\sum_{i=1}^{K}\left|\mathbf{h}_{0}^{\mathcal{H}} \mathbf{q}_{i}\right|^{2}\right] \leq Q_{a v} .
\end{aligned}
$$

It is easy to verify that Problem (5) is non-convex. Thus, finding the global optimum is challenging. However, there are a number of existing algorithms for globally optimizing non-CR WSRMax problems, such as, branch-and-bound algorithms introduced in [11][12][13], a monotonic optimization approach proposed in [14] and a decentralized algorithm derived in [31]. Other approaches, such as a semi-distributed algorithm and an alternative centralized algorithm introduced by [8] (for solving CR MIMO Ad Hoc WSRMax problems), successive convex approximation with second-order cone programming derived by [16], as well as a ZGIM algorithm proposed by [15][7], can also be employed to obtain a local optimum for Problem (5). As mentioned earlier, we use our own 'BF-LDP-ZGIM' algorithm (adapted from ZGIM for solving Problem (5)) (see Section III-A of [17] for further details) as a benchmark for performance comparison of our proposed suboptimal algorithms. In general, there is no closed form solution for Problem (5), and although we can numerically obtain globally/locally optimum BF using adaptations of the above algorithms, they have high computational complexity and are not amenable to further analysis of the BF solution. Motivated by this, in the next section, we will focus on deriving low-complexity albeit suboptimal BF strategies for Problem (5) with a simple explicit BF solution.

\section{Suboptimum Beamforming Design}

The beamforming vector $\mathbf{q}_{i}, \forall i=1, \ldots, K$ also can be expressed as $\mathbf{q}_{i}=\sqrt{p_{i}} \mathbf{g}_{i}$, where $p_{i}$ and $\mathbf{g}_{i}$ are the transmit signal power and the beamforming weight vector for $\mathrm{SU}_{i}$ respectively. 
A. Partially-projected and Regularized Zero-forcing Beamforming $(P P-R Z F B F)$

Let $\mathbf{G} \triangleq\left\{\mathbf{g}_{1}, \ldots, \mathbf{g}_{K}\right\} \in \mathbb{C}^{M \times K}$ denote the beamforming weight matrix. Similar to [10], we first look at two extreme cases of Problem (5):

Case I (High $\left.Q_{a v}\right)$ : In this scenario, we assume that $Q_{a v}$ is large enough to make the AIP constraint inactive. Problem (5) reduces to the conventional non-CR MISO-BC WSRMax problem with the ATTP constraint only. A popular and effective suboptimal scheme for this conventional scenario is known as zero-forcing beamforming (ZFBF)[20], where the $\mathrm{BF}$ vectors are designed to completely remove the multiuser interference. One straightforward choice of such beamforming matrix, denoted as $\mathbf{G}_{\mathrm{I}}$, is the pseudo-inverse of $\mathbf{H}^{\mathcal{H}}$, i.e., $\mathbf{G}_{\mathrm{I}}=\mathbf{H}\left(\mathbf{H}^{\mathcal{H}} \mathbf{H}\right)^{-1}$. However, as pointed out by [20], ZFBF has some shortcomings, such as, the inverse of $\mathbf{H}^{\mathcal{H}} \mathbf{H}$ may not exist and when $K=M$, the sum-rate of ZFBF does not scale linearly with $K$ (or $M$ ). These drawbacks can be improved by adding a regularization term before inverting, i.e., $\mathbf{G}_{\mathbf{I}}=\mathbf{H}\left(\mathbf{H}^{\mathcal{H}} \mathbf{H}+\alpha \mathbf{I}\right)^{-1} \stackrel{(a)}{=}\left(\mathbf{H H}^{\mathcal{H}}+\alpha \mathbf{I}\right)^{-1} \mathbf{H}$, known as regularized zero-forcing beamforming (RZFBF). $\alpha>0$ is the regularization parameter which controls the amount of multiuser interference, and (a) is an equivalent expression as given in [21][22].

Case II $\left(Q_{a v}=0\right)$ : In this case, the AIP constraint in Problem (5) becomes $\mathbf{h}_{0}^{\mathcal{H}} \mathbf{g}_{i}=0, \forall i=1, \ldots, K$, which implies zero interference on the PU caused by the secondary network. For this scenario, by applying the "channel projection" idea introduced by [2][10], we first project $\mathbf{H}$ into the null space of $\mathbf{h}_{0}$, given as $\mathbf{H}_{\perp}=\left(\mathbf{I}-\overline{\mathbf{h}}_{0} \overline{\mathbf{h}}_{0}^{\mathcal{H}}\right) \mathbf{H}$, where $\overline{\mathbf{h}}_{0}=\frac{\mathbf{h}_{0}}{\left\|\mathbf{h}_{0}\right\|}$, and then design the beamforming matrix, denoted as $\mathbf{G}_{\mathrm{II}}$, based on the projected channel matrix $\mathbf{H}_{\perp}$, so that the constraints $\mathbf{h}_{0}^{\mathcal{H}} \mathbf{g}_{i}=0, \forall i=1, \ldots, K$ are guaranteed to meet. More specifically, we have $\mathbf{H}=\mathbf{H}_{\perp}+\overline{\mathbf{h}}_{0} \overline{\mathbf{h}}_{0}^{\mathcal{H}} \mathbf{H}$ and $\mathbf{h}_{0}^{\mathcal{H}} \mathbf{H}_{\perp}=\mathbf{0}$, which gives $\mathbf{H}^{\mathcal{H}} \mathbf{g}_{i}=\mathbf{H}_{\perp}^{\mathcal{H}} \mathbf{g}_{i}, \forall i=1, \ldots, K$. Applying this into (5), Problem (5) again becomes a conventional nonCR MISO-BC WSRMax problem with channel matrix $\mathbf{H}_{\perp}$, where according to the Case I, suboptimal RZFBF scheme can be applied to design the beamforming matrix, given as $\mathbf{G}_{\mathrm{II}}=\mathbf{H}_{\perp}\left(\mathbf{H}_{\perp}^{\mathcal{H}} \mathbf{H}_{\perp}+\alpha \mathbf{I}\right)^{-1}=\left(\mathbf{H}_{\perp} \mathbf{H}_{\perp}^{\mathcal{H}}+\alpha \mathbf{I}\right)^{-1} \mathbf{H}_{\perp}$. Obviously, we have $\mathbf{h}_{0}^{\mathcal{H}} \mathbf{G}_{\text {II }}=0$, i.e., $\mathbf{h}_{0}^{\mathcal{H}} \mathbf{g}_{i}=0, \forall i=1, \ldots, K$.

Remark 2: From the definition of $\mathbf{H}_{\perp}$, we have $\operatorname{rank}\left(\mathbf{H}_{\perp}\right) \leq \min \left[\operatorname{rank}\left(\mathbf{I}-\overline{\mathbf{h}}_{0} \overline{\mathbf{h}}_{0}^{\mathcal{H}}\right), \operatorname{rank}(\mathbf{H})\right]$. Since $\operatorname{rank}\left(\mathbf{I}-\overline{\mathbf{h}}_{0} \overline{\mathbf{h}}_{0}^{\mathcal{H}}\right)=M-1$ and $\operatorname{rank}(\mathbf{H}) \leq \min (M, K)$, thus we can obtain $\operatorname{rank}\left(\mathbf{H}_{\perp}\right) \leq \min (M-1, K)<M$. When $K \geq M, \operatorname{Rank}\left(\mathbf{H}_{\perp}\right)<\operatorname{Rank}(\mathbf{H})$ as a result of orthogonality to $\mathbf{h}_{0}$, and both $\mathbf{H}_{\perp} \mathbf{H}_{\perp}^{\mathcal{H}}$ and $\mathbf{H}_{\perp}^{\mathcal{H}} \mathbf{H}_{\perp}$ are rank-deficient and not invertible, which thus requires $\alpha \neq 0$.

In the two special cases mentioned above, the beamforming matrix is designed by applying the RZFBF scheme based on a certain form of $\mathbf{H}$. By carefully comparing the expressions of $\mathbf{G}_{\mathrm{I}}$ and $\mathbf{G}_{\mathrm{II}}$, we observe that as $Q_{a v}$ becomes smaller, the certain form of $\mathbf{H}$ mentioned above used for beamformer design changes from $\mathbf{H}$ to $\mathbf{H}_{\perp}$. It is seen that as $Q_{a v}$ decreases, the beamformer design evolves along a sequence of subspaces of $\mathbf{H}$ with increasing amounts of projection into the null space of $\mathbf{h}_{0}$ [10]. This motivates a general heuristic suboptimal method for Problem (5) for any given $Q_{a v}$, by combining RZF [20] (to control secondary multiuser interference) with the 'channel projection' idea [2][10][9] (to control the secondary interference on PU), in order to achieve a tradeoff between maximizing secondary throughput and suppressing secondary multiuser interference as well as the interference on PU. We call it the partially-projected-RZFBF (PP-RZFBF) scheme. In this scheme, first, similar to [2][10][9], the secondary channel matrix $\mathbf{H}$ is partially projected into the null space of $\mathbf{h}_{0}$, denoted as $\tilde{\mathbf{H}}$ and given as [9], $\tilde{\mathbf{H}}=\left(\mathbf{I}-\beta \overline{\mathbf{h}}_{0} \overline{\mathbf{h}}_{0}^{\mathcal{H}}\right) \mathbf{H}$, where $0 \leq \beta \leq 1$ is the projection control parameter; then RZFBF algorithm is applied to $\tilde{\mathbf{H}}$ to obtain the beamforming matrix, namely,

$$
\mathbf{G}=\tilde{\mathbf{H}}\left(\tilde{\mathbf{H}}^{\mathcal{H}} \tilde{\mathbf{H}}+\alpha \mathbf{I}\right)^{-1}=\left(\tilde{\mathbf{H}} \tilde{\mathbf{H}}^{\mathcal{H}}+\alpha \mathbf{I}\right)^{-1} \tilde{\mathbf{H}},
$$

which gives, $\mathbf{g}_{i}=\left(\tilde{\mathbf{H}} \tilde{\mathbf{H}}^{\mathcal{H}}+\alpha \mathbf{I}\right)^{-1} \tilde{\mathbf{h}}_{i}, \forall i=1, \ldots, K$ where the vector $\tilde{\mathbf{h}}_{i}$ is the $i^{t h}$ column of $\tilde{\mathbf{H}}$. Obviously, $\beta=0$ and $\beta=1$ correspond to Case I and Case II, respectively.

Thus, let $\tilde{\mathbf{H}}_{-i} \triangleq\left\{\tilde{\mathbf{h}}_{1}, \ldots, \tilde{\mathbf{h}}_{i-1}, \tilde{\mathbf{h}}_{i+1}, \ldots, \tilde{\mathbf{h}}_{K}\right\} \in \mathcal{C}^{M \times K-1}$ and $\mathbf{P}_{-i} \triangleq \operatorname{diag}\left(p_{1}, \ldots, p_{i-1}, p_{i+1}, \ldots, p_{K}\right)$, Problem (5) with the suboptimal PP-RZFBF scheme can be reduced to,

$\underset{0 \leq \beta \leq 1, \alpha>0,\left\{p_{i} \geq 0\right\}_{i=1}^{K}}{\operatorname{maximize}} R_{\mathrm{PP}-\mathrm{RZFBF}}=\sum_{i=1}^{K} E\left[w_{i} \log \left(1+\operatorname{SINR}_{i}\right)\right]$

$$
\text { subject to } \quad E[\phi] \leq P_{a v}, \quad E[\psi] \leq Q_{a v},
$$

where

$$
\begin{aligned}
\operatorname{SINR}_{i} & =\frac{p_{i}\left|\mathbf{h}_{i}^{\mathcal{H}}\left(\tilde{\mathbf{H}} \tilde{\mathbf{H}}^{\mathcal{H}}+\alpha \mathbf{I}\right)^{-1} \tilde{\mathbf{h}}_{i}\right|^{2}}{1+\mathbf{h}_{i}^{\mathcal{H}}\left(\tilde{\mathbf{H}} \tilde{\mathbf{H}}^{\mathcal{H}}+\alpha \mathbf{I}\right)^{-1} \tilde{\mathbf{H}}_{-i} \mathbf{P}_{-i} \tilde{\mathbf{H}}_{-i}^{\mathcal{H}}\left(\tilde{\mathbf{H}} \tilde{\mathbf{H}}^{\mathcal{H}}+\alpha \mathbf{I}\right)^{-1} \mathbf{h}_{i}}, \\
\phi & \triangleq \sum_{i=1}^{K} p_{i} \tilde{\mathbf{h}}_{i}^{\mathcal{H}}\left(\tilde{\mathbf{H}} \tilde{\mathbf{H}}^{\mathcal{H}}+\alpha \mathbf{I}\right)^{-2} \tilde{\mathbf{h}}_{i}, \\
\psi & \triangleq \sum_{i=1}^{K} p_{i}\left|\mathbf{h}_{0}^{\mathcal{H}}\left(\tilde{\mathbf{H}} \tilde{\mathbf{H}}^{\mathcal{H}}+\alpha \mathbf{I}\right)^{-1} \tilde{\mathbf{h}}_{i}\right|^{2} .
\end{aligned}
$$

Although its complexity is largely reduced compared to Problem (5), Problem (7) is still a non-convex optimization problem. However, for given $\beta$ and $\alpha$, a locally optimal power allocation scheme can be obtained by using the SCALE algorithm proposed by [32]. Then, we can utilize a twodimensional exhaustive search method to get the optimal $\beta$ and $\alpha$, in order to obtain a locally optimal PP-RZFBF beamformer. Obviously, a simultaneous exhaustive search over both $\beta$ and $\alpha$ is computationally prohibitive, especially since the searching range for $\alpha$ is from 0 to $\infty$. This motivates us to do a large system analysis in Section IV, which enables us to derive simple but asymptotically optimal values of $\beta^{*}$ and $\alpha^{*}$.

Remark 3: When $\beta=1$, $\tilde{\mathbf{H}}$ becomes $\mathbf{H}_{\perp}$, which, as we mentioned before, is orthogonal to $\mathbf{h}_{0}$ and gives $\mathbf{h}_{0}^{\mathcal{H}} \mathbf{g}_{i}=$ $0, \forall i=1, \ldots, K$, i.e., zero interference on the PU. Therefore, in Problem (7), when $\beta=1$, the AIP constraint is always inactive unless $Q_{a v}=0$.

\section{B. Complete Zero-forcing Beamforming (CZFBF)}

Zero interference based suboptimal BF design idea has been extended to the CR network, for example, in [33], a ZF block diagonalization precoder is developed for a CR MIMO BC network. Here, we will also consider a ZF based suboptimal 
algorithm for Problem (5), as a performance benchmark for PP-RZFBF, by zero forcing all the interference (including the secondary multiuser interference as well as the interference imposed on PU due to the secondary transmission), where each beamforming vectors $\mathbf{g}_{i}, \forall i=1, \ldots, K$, satisfies $\mathbf{h}_{j}^{\mathcal{H}} \mathbf{g}_{i}=$ $0, \forall j \neq i$, and $\mathbf{h}_{0}^{\mathcal{H}} \mathbf{g}_{i}=0$. We call this special suboptimal ZF solution as the "Complete-ZFBF (CZFBF)" scheme. Let $\mathbf{F}_{-i} \triangleq\left\{\mathbf{h}_{0}, \mathbf{h}_{1}, \ldots, \mathbf{h}_{i-1}, \mathbf{h}_{i+1}, \ldots, \mathbf{h}_{K}\right\} \in \mathbb{C}^{M \times K}$, then the above ZF criteria can be rewritten as $\mathbf{F}_{-i}^{\mathcal{H}} \mathbf{g}_{i}=\mathbf{0}$, thus $\mathbf{g}_{i}$ is in the null space of the matrix $\mathbf{F}_{-i}^{\mathcal{H}}$.

1) When $K<M, \operatorname{rank}\left(\mathbf{F}_{-i}^{\mathcal{H}}\right)<M$, and thus $\mathbf{F}_{-i}^{\mathcal{H}} \mathbf{g}_{i}=\mathbf{0}, \forall i$ has an infinite number of solutions. One such solution can be chosen for the CZFBF scheme as $\mathbf{G}=\mathbf{H}_{\perp}\left(\mathbf{H}_{\perp}^{\mathcal{H}} \mathbf{H}_{\perp}\right)^{-1}$, where $\mathbf{H}_{\perp}=\left(\mathbf{I}-\overline{\mathbf{h}}_{0} \overline{\mathbf{h}}_{0}^{\mathcal{H}}\right) \mathbf{H}$.

Remark 4: Comparing the CZFBF and the PP-RZFBF beamforming matrices, it is seen that the CZFBF scheme for $K<M$ case obviously corresponds to the PP-RZFBF scheme with $\beta=1$ and $\alpha=0$. We will show later in Section IV that a large system analysis can be used to obtain an asymptotically optimal beamformer with $\alpha^{*}=\frac{K}{P_{a v}}$ and a range of values for the optimal $\beta^{*}$. Then the resulting $\alpha^{*}$ and $\beta^{*}$ can be applied in our proposed PP-RZFBF scheme to obtain a low-complexity suboptimal beamformer (called PP-RZFBF-ABF) for finitesized CR-MISO-BC systems. According to Proposition 6, in the large system limit, as $P_{a v} \rightarrow \infty$, the set of optimal $\beta^{*}$ collapses to just one element ' 1 '. Thus, in high $P_{a v}, \alpha^{*}=\frac{K}{P_{a v}}$ and $\beta^{*} \rightarrow 1$ can be applied to obtain the PP-RZFBF-ABF scheme for use in the finite CR-MISO-BC systems. It follows then that, for given finite values of $K$ and $M$ with $K<M$, the PP-RZFBF-ABF scheme approaches the CZFBF scheme as $P_{a v} \rightarrow \infty$.

2) When $K \geq M, \operatorname{rank}\left(\mathbf{F}_{-i}^{\mathcal{H}}\right)=M$, thus $\mathbf{F}_{-i}^{\mathcal{H}} \mathbf{g}_{i}=\mathbf{0}$ only has a trivial solution, i.e., $\mathbf{g}_{i}=\mathbf{0}$. In this case, all the beamforming vectors are zero vectors, implying that the CRBS is not transmitting. To avoid this, a user selection algorithm needs to be utilized to make sure that at most $M-1$ out of the $K$ users are active (or at least $K-M+1$ number of users must be inactive), so that a non-trivial CZFBF beamformer can be obtained. In other words, when $K \geq M$, CR-BS can only transmit to a subset of fewer than $K$ SUs to achieve zero forcing on all resulting interference. Note that designing an optimal user scheduling algorithm is beyond the scope of this paper. However, since all the SU channels are assumed to be i.i.d, in the case when all weights are the same, we can just randomly choose $M-1$ SUs without sacrificing fairness.

Remark 5: When $K \geq M$, according to Remark 2, if $\beta=1, \alpha$ must be nonzero for the PP-RZFBF scheme. Since it does not implement user selection, the PP-RZFBF scheme with $\beta \rightarrow 1$ and $\alpha \rightarrow 0$ does not lead to the CZFBF scheme for $K \geq M$ case.

Without loss of generality, let $\mathcal{S} \subseteq\{1, \ldots, K\}$ be the selected subset of SUs indexes with $|\mathcal{S}|=\min (M-1, K)$. The CZFBF beamforming matrix can be chosen as $\mathbf{G}=\mathbf{H}_{\perp}^{s}\left(\mathbf{H}_{\perp}^{s} \mathcal{H} \mathbf{H}_{\perp}^{s}\right)^{-1}$, where $\mathbf{H}_{\perp}^{s}=\left(\mathbf{I}-\overline{\mathbf{h}}_{0} \overline{\mathbf{h}}_{0}^{\mathcal{H}}\right) \mathbf{H}^{s}$ with $\mathbf{H}^{s}=\left\{h_{i}, \forall i \in \mathcal{S}\right\}$. Problem
(5) with the CZFBF scheme becomes,

$$
\begin{array}{ll}
\operatorname{maximize}_{p_{i} \geq 0, \forall i \in \mathcal{S}} & R_{\mathrm{CZFBF}}=\sum_{i \in \mathcal{S}} w_{i} E\left[\log \left(1+p_{i}\left|\mathbf{h}_{i}^{\mathcal{H}} \mathbf{g}_{i}\right|^{2}\right)\right] \\
\text { subject to } & E\left[\sum_{i \in \mathcal{S}} p_{i}\left|\mathbf{g}_{i}\right|^{2}\right] \leq P_{a v} .
\end{array}
$$

The optimal power allocation for CZFBF scheme is given by water-filling, i.e., $p_{i}=\left(\frac{w_{i}}{\lambda_{0}\left|\mathbf{g}_{i}\right|^{2}}-\frac{1}{\left|\mathbf{h}_{i}^{\mathcal{H}} \mathbf{g}_{i}\right|^{2}}\right)^{+}, \quad i \in$ $\mathcal{S}$, where the Lagrange multiplier $\lambda_{0}$ is determined by $E\left[\sum_{i \in \mathcal{S}} p_{i}\left|\mathbf{g}_{i}\right|^{2}\right]=P_{a v}$.

\section{LARGE SYSTEM ANALYSIS}

Finding the optimal design parameters (i.e., $\alpha, \beta$ ) for PP-RZFBF (Problem (7)) with a two-dimensional bruteforce search method is prohibitively time-consuming. In this section, we will pursue a large system analysis of the PP-RZFBF scheme, in the limit as $M$ and $K$ grow jointly to infinity with a fixed ratio $r=\frac{K}{M}$, defined as the "large system limit". Motivated by existing work in [21][22][23][25] (large system analysis for the non-CR MISO-BC scenario) and [26] (large system analysis for a non-CR multi-cell downlink cooperation system), we will show that in the large system limit, $\operatorname{SINR}_{i}, \phi, \psi$ in Problem (7) converge almost surely to deterministic approximations, denoted by $\operatorname{SINR}_{i}^{\infty}, \phi^{\infty}, \psi^{\infty}$, respectively. This will later enable us to determine the asymptotically optimal design parameters $\alpha, \beta$ for Problem (7) and get further insight into the asymptotical behaviour of our CR-MISO-BC system.

In the large system limit, we assume that $\max \left(p_{1}, \ldots, p_{K}\right)=\mathcal{O}\left(\frac{1}{K}\right)$. And note that according to [21][27], since $\mathbf{H}$ is $M \times K$ with i.i.d standardized complex Gaussian entries (i.e., having zero mean and unit variance), $\frac{1}{M} \mathbf{H} \mathbf{H}^{\mathcal{H}}$ has a uniformly bounded spectral norm for all large $M$. Define $\overline{\mathbf{W}} \triangleq \mathbf{I}-\beta \overline{\mathbf{h}}_{0} \overline{\mathbf{h}}_{0}^{\mathcal{H}}$ and let $\hat{\mathbf{h}}_{0}=\frac{1}{\sqrt{M}} \mathbf{h}_{0}, \hat{\mathbf{h}}_{i}=\frac{1}{\sqrt{M}} \mathbf{h}_{i}, \forall i=1, \ldots, K$ and $\hat{\mathbf{H}}_{-i} \triangleq\left\{\hat{\mathbf{h}}_{1}, \ldots, \hat{\mathbf{h}}_{i-1}, \hat{\mathbf{h}}_{i+1}, \ldots, \hat{\mathbf{h}}_{K}\right\} \in \mathcal{C}^{M \times K-1}$. Define $\Gamma_{-i} \triangleq\left(\frac{\tilde{\mathbf{H}}_{-i} \tilde{\mathbf{H}}_{-i}^{\mathcal{H}}}{M}+\alpha_{0} \mathbf{I}\right)$, where $\alpha_{0}=\frac{\alpha}{M}$. Since $\frac{1}{\sqrt{M}} \tilde{\mathbf{H}}_{-i}=\overline{\mathbf{W}} \hat{\mathbf{H}}_{-i}$, we have $\Gamma_{-i}=\left(\overline{\mathbf{W}} \hat{\mathbf{H}}_{-i} \hat{\mathbf{H}}_{-i}^{\mathcal{H}} \overline{\mathbf{W}}+\alpha_{0} \mathbf{I}\right)$. Then we can obtain following result:

Proposition 2: As $M \rightarrow \infty, K \rightarrow \infty$, with a fixed ratio $r=\frac{K}{M}$, we have,

$\frac{1}{M} \operatorname{tr}\left\{\Gamma_{-i}^{-1}\right\} \rightarrow z\left(r, \alpha_{0}\right)$,

$\hat{\mathbf{h}}_{0}^{\mathcal{H}} \Gamma_{-i}^{-1} \hat{\mathbf{h}}_{0} \rightarrow \frac{z\left(r, \alpha_{0}\right)}{1+\left(\beta^{2}-2 \beta\right)\left(1-\alpha_{0} z\left(r, \alpha_{0}\right)\right)}$,

$\frac{\partial \hat{\mathbf{h}}_{0}^{\mathcal{H}} \Gamma_{-i}^{-1} \hat{\mathbf{h}}_{0}}{\partial \alpha_{0}} \rightarrow$

$-\frac{z\left(r, \alpha_{0}\right)\left(1+z\left(r, \alpha_{0}\right)\right)^{2}+z\left(r, \alpha_{0}\right)^{3} r\left(\beta^{2}-2 \beta\right)}{\left(1+\left(\beta^{2}-2 \beta\right)\left(1-\alpha_{0} z\left(r, \alpha_{0}\right)\right)\right)^{2}\left(r+\alpha_{0}\left(1+z\left(r, \alpha_{0}\right)\right)^{2}\right)}$,

almost $\quad$ surely,
$\frac{1}{2}\left[\sqrt{\frac{(1-r)^{2}}{\alpha_{0}^{2}}+\frac{2(1+r)}{\alpha_{0}}+1}+\frac{1-r}{\alpha_{0}}-1\right]$. 
Proof: See Appendix B.

Remark 6: From Proposition 2, we find that $\frac{1}{M} \operatorname{tr}\left\{\Gamma_{-i}^{-1}\right\}=$ $\frac{1}{M} \operatorname{tr}\left(\overline{\mathbf{W}} \hat{\mathbf{H}}_{-i} \hat{\mathbf{H}}_{-i}^{\mathcal{H}} \overline{\mathbf{W}}+\alpha_{0} \mathbf{I}\right) \quad\left(\right.$ where $\overline{\mathbf{W}} \triangleq \mathbf{I}-\beta \overline{\mathbf{h}}_{0} \overline{\mathbf{h}}_{0}^{\mathcal{H}}$ ) attains the same deterministic asymptote $z\left(r, \alpha_{0}\right)$ as $\frac{1}{M} \operatorname{tr}\left(\hat{\mathbf{H}}_{-i} \hat{\mathbf{H}}_{-i}^{\mathcal{H}}+\alpha_{0} \mathbf{I}\right)$, i.e., $\beta \overline{\mathbf{h}}_{0} \overline{\mathbf{h}}_{0}^{\mathcal{H}}$ does not affect the asymptotic value of $\frac{1}{M} \operatorname{tr}\left\{\Gamma_{-i}^{-1}\right\}$. This is attributed to the fact that for $\frac{1}{M} \operatorname{tr}\left\{\Gamma_{-i}^{-1}\right\}, \beta \overline{\mathbf{h}}_{0} \overline{\mathbf{h}}_{0}^{\mathcal{H}}$ is like a rank-one perturbation of a large matrix and its impact vanishes asymptotically as $M \rightarrow \infty$. On the contrary, $\beta \overline{\mathbf{h}}_{0} \overline{\mathbf{h}}_{0}^{\mathcal{H}}$ inside $\Gamma_{-i}$ has a greater impact on the asymptotic value of $\hat{\mathbf{h}}_{0}^{\mathcal{H}} \Gamma_{-i}^{-1} \hat{\mathbf{h}}_{0}$, since it is highly correlated with $\hat{\mathbf{h}}_{0}$. For example, when $\beta=1$, we have $\hat{\mathbf{h}}_{0}^{\mathcal{H}} \Gamma_{-i}^{-1} \hat{\mathbf{h}}_{0} \rightarrow \frac{1}{\alpha_{0}}$; whereas, when $\beta=0$, we have $\hat{\mathbf{h}}_{0}^{\mathcal{H}} \Gamma_{-i}^{-1} \hat{\mathbf{h}}_{0} \rightarrow z\left(r, \alpha_{0}\right)$.

Based on Proposition 2, the asymptotic expressions of $\operatorname{SINR}_{i}, \phi, \psi$ can be obtained as follows:

Theorem 1: Let $\alpha_{0}>0$. Then as $M \rightarrow \infty, K \rightarrow \infty$ with fixed $r=\frac{K}{M}<\infty$,

$$
\begin{aligned}
\operatorname{SINR}_{i} & \stackrel{\text { a.s. }}{\rightarrow} \operatorname{SINR}_{i}^{\infty} \\
& =\frac{p_{i} z\left(r, \alpha_{0}\right)^{2}}{\left(1+z\left(r, \alpha_{0}\right)\right)^{2}+\tau\left(r, \alpha_{0}\right)\left(\frac{1}{K} \sum_{j=1}^{K} p_{j}-\frac{p_{i}}{K}\right)} \\
\phi & \stackrel{\text { a.s. }}{\rightarrow} \phi^{\infty}=\tau\left(r, \alpha_{0}\right) \frac{1}{K} \sum_{i=1}^{K} p_{i} \\
\psi & \stackrel{\text { a.s. }}{\rightarrow} \psi^{\infty}=\rho\left(r, \alpha_{0}, \beta\right) \tau\left(r, \alpha_{0}\right) \frac{1}{K} \sum_{i=1}^{K} p_{i}
\end{aligned}
$$

where $\tau\left(r, \alpha_{0}\right) \triangleq \frac{r z\left(r, \alpha_{0}\right)}{r+\alpha_{0}\left(1+z\left(r, \alpha_{0}\right)\right)^{2}}$ and $\rho\left(r, \alpha_{0}, \beta\right) \triangleq$ $\frac{(1-\beta)^{2}}{\left(1+\left(\beta^{2}-2 \beta\right)\left(1-\alpha_{0} z\left(r, \alpha_{0}\right)\right)\right)^{2}}$.

Proof: See Appendix C.

Remark 7: Theorem 1 illustrates that $\operatorname{SINR}_{i}^{\infty}, \phi^{\infty}, \psi^{\infty}$ are all deterministic quantities and do not depend on any instantaneous CSIT $\left(\mathbf{H}, \mathbf{h}_{0}\right)$. Furthermore, from Theorem 1, when $\beta=0$, we have $\psi^{\infty}=\phi^{\infty}$ and the expressions of $\operatorname{SINR}_{i}^{\infty}$, and $\phi^{\infty}$ in (11a)(11b) reduce to that of the corresponding non$\mathrm{CR}$ case considered in [21][22][23][25]. In case of $\beta=1$, we have $\psi^{\infty}=0$, i.e., the interference on PU caused by the secondary transmission is nullified, which is consistent with Remark 3.

Note that with the assumption $\max \left(p_{1}, \ldots, p_{K}\right)=\mathcal{O}\left(\frac{1}{K}\right)$, the term $\frac{p_{i}}{K}$ in (11a) can be omitted. Therefore, with the assumption $\sum_{i}^{K} w_{i}=\mathcal{O}(1)$, as $M \rightarrow \infty, K \rightarrow \infty$ with their ratio $r$ fixed, Problem (7) becomes,

$$
\begin{array}{cl}
\underset{\beta, \alpha_{0},\left\{p_{i}\right\}_{i=1}^{K}}{\operatorname{maximize}} & R_{\mathrm{PP}-\mathrm{RZFBF}}^{\infty}=\sum_{i=1}^{K} w_{i} \log \left(1+p_{i} v_{0}\left(r, \alpha_{0}, \beta\right)\right) \\
\text { subject to } & \frac{1}{K} \sum_{i=1}^{K} p_{i}=\bar{P}\left(r, \alpha_{0}, \beta\right), \\
& 0 \leq \beta \leq 1, \alpha_{0}>0, p_{i} \geq 0, \forall i=1, \ldots, K
\end{array}
$$

where

$$
\begin{aligned}
& \bar{P}\left(r, \alpha_{0}, \beta\right)= \\
& \begin{cases}\min \left\{\frac{P_{a v}}{\tau\left(r, \alpha_{0}\right)}, \frac{Q_{a v}}{\rho\left(r, \alpha_{0}, \beta\right) \tau\left(r, \alpha_{0}\right)}\right\}, & \text { when } \rho\left(r, \alpha_{0}, \beta\right) \neq 0 ; \\
\frac{P_{a v}}{\tau\left(r, \alpha_{0}\right)}, & \text { when } \rho\left(r, \alpha_{0}, \beta\right)=0,\end{cases}
\end{aligned}
$$

and $v_{0}\left(r, \alpha_{0}, \beta\right) \triangleq \frac{z\left(r, \alpha_{0}\right)^{2}}{\left(1+z\left(r, \alpha_{0}\right)\right)^{2}+\tau\left(r, \alpha_{0}\right) \frac{1}{K} \sum_{j=1}^{K} p_{j}} \stackrel{(a)}{=}$ $\frac{z\left(r, \alpha_{0}\right)^{2}}{}$, where (a) is obtained by applying the power constraint of Problem (12).

\section{A. Asymptotically Optimal beamforming for PP-RZFBF Scheme (PP-RZFBF-ABF)}

In this subsection, we will find the asymptotically optimal design parameters for PP-RZFBF Scheme by optimally solving Problem (12). Problem (12) can be shown to be equivalent to the following problem (the proof is similar to Proposition 1 of [34] and is omitted):

$$
\underset{0 \leq \beta \leq 1, \alpha_{0}>0}{\operatorname{maximize}} f\left(\beta, \alpha_{0}\right)
$$

where

$$
\begin{aligned}
& f\left(\beta, \alpha_{0}\right) \triangleq \operatorname{maximize}_{\left\{p_{i} \geq 0\right\}_{i=1}^{K}} \sum_{i=1}^{K} w_{i} \log \left(1+p_{i} v_{0}\left(r, \alpha_{0}, \beta\right)\right) \\
& \text { subject to } \frac{1}{K} \sum_{i=1}^{K} p_{i}=\bar{P}\left(r, \alpha_{0}, \beta\right) \text {, }
\end{aligned}
$$

With fixed $\beta$ and $\alpha_{0}$, Problem (15) is a convex optimization problem and the optimal $\left\{p_{i}\right\}_{i=1}^{K}$ is the conventional waterfilling,

$$
p_{i}^{*}=\left[\frac{w_{i}}{\lambda_{\infty}}-\frac{1}{v_{0}\left(r, \alpha_{0}, \beta\right)}\right]^{+}, \quad \forall i=1, \ldots, K
$$

where $\lambda_{\infty}$ is the Lagrange multiplier determined by the constraint $\frac{1}{K} \sum_{i=1}^{K} p_{i}=\bar{P}\left(r, \alpha_{0}, \beta\right)$. Without loss of generality, assuming $w_{1} \geq \cdots \geq w_{K}$, from (16), we obtain $p_{1} \geq \cdots \geq p_{K}$. Assuming there are $\bar{K}$ non-zero powers we can obtain $\lambda_{\infty}=\frac{\frac{1}{K} \sum_{i=1}^{K} w_{i}}{\frac{1}{K} \frac{1}{v_{0}\left(r, \alpha_{0}, \beta\right)}+\bar{P}\left(r, \alpha_{0}, \beta\right)}$. Note that in the case of $w_{1}=\cdots=w_{K}=w$, we have equal power allocation for each SU, given by $p_{1}=\cdots=p_{K}=\bar{P}\left(r, \alpha_{0}, \beta\right)$, and the corresponding $R_{\mathrm{PP}-\mathrm{RZFBF}}^{\infty}=K w \log \left(1+\bar{P}\left(r, \alpha_{0}, \beta\right) v_{0}\left(r, \alpha_{0}, \beta\right)\right)$.

Substituting (16) into (15), Problem (14) can be expressed as

$$
\begin{aligned}
\underset{0 \leq \beta \leq 1, \alpha_{0}>0}{\operatorname{maximize}} & \sum_{i=1}^{\bar{K}} w_{i} \log \left(\frac{\bar{K}}{K}+\bar{P}\left(r, \alpha_{0}, \beta\right) v_{0}\left(r, \alpha_{0}, \beta\right)\right) \\
& +\sum_{i=1}^{\bar{K}} w_{i} \log \left(\frac{w_{i}}{\frac{1}{K} \sum_{i=1}^{\bar{K}} w_{i}}\right)
\end{aligned}
$$

which is equivalent to the following optimization problem:

$$
\begin{aligned}
\underset{0 \leq \beta \leq 1, \alpha_{0}>0}{\operatorname{maximize}} & \bar{P}\left(r, \alpha_{0}, \beta\right) v_{0}\left(r, \alpha_{0}, \beta\right) \\
& =\frac{\bar{P}\left(r, \alpha_{0}, \beta\right) z\left(r, \alpha_{0}\right)^{2}}{\left(1+z\left(r, \alpha_{0}\right)\right)^{2}+\tau\left(r, \alpha_{0}\right) \bar{P}\left(r, \alpha_{0}, \beta\right)} .
\end{aligned}
$$


Discussions:

Case 1: If $\rho\left(r, \alpha_{0}, \beta\right)<\frac{Q_{a v}}{P_{a v}}$, then from (13), we must have $\bar{P}\left(r, \alpha_{0}, \beta\right)=\frac{P_{a v}}{\tau\left(r, \alpha_{0}\right)}$, implying that only the ATTP constraint is active. In this scenario, the objective function of Problem (18) becomes, $\bar{P}\left(r, \alpha_{0}, \beta\right) v_{0}\left(r, \alpha_{0}, \beta\right)=\frac{\frac{P P_{a v}}{\tau\left(r, \alpha_{0}\right)} z\left(r, \alpha_{0}\right)^{2}}{\left(1+z\left(r, \alpha_{0}\right)\right)^{2}+P_{a v}}$, which is independent of $\beta$. Thus the optimal $\beta$ of Problem (18) only needs to satisfy

$$
0 \leq \beta \leq 1 \text { and } \rho\left(r, \alpha_{0}, \beta\right)<\frac{Q_{a v}}{P_{a v}} .
$$

Proposition 3: Let $t=\sqrt{\frac{Q_{a v}}{P_{a v}}}$. Define the set $\mathcal{A}_{1} \triangleq$ $\left\{\beta \mid 0 \leq \beta \leq 1 \& \rho\left(r, \alpha_{0}, \beta\right)<\frac{Q_{a v}}{P_{a v}}\right\}$. Then the set $\mathcal{A}_{1}$ can be rewritten as (20) (shown on the top of the next page), where

$$
\begin{aligned}
\phi_{1}\left(r, \alpha_{0}\right) \triangleq & \left(\sqrt{1-\frac{1}{1-\alpha_{0} z\left(r, \alpha_{0}\right)}+\frac{1}{4 t^{2}\left(1-\alpha_{0} z\left(r, \alpha_{0}\right)\right)^{2}}}\right. \\
& \left.-\frac{1}{2 t\left(1-\alpha_{0} z\left(r, \alpha_{0}\right)\right)}+1\right), \\
\phi_{2}\left(r, \alpha_{0}\right) \triangleq & \left(-\sqrt{1-\frac{1}{1-\alpha_{0} z\left(r, \alpha_{0}\right)}+\frac{1}{4 t^{2}\left(1-\alpha_{0} z\left(r, \alpha_{0}\right)\right)^{2}}}\right. \\
& \left.-\frac{1}{2 t\left(1-\alpha_{0} z\left(r, \alpha_{0}\right)\right)}+1\right) .
\end{aligned}
$$

Proof: See Appendix D.

Therefore, under Case 1, the optimal solution for Problem (18), denoted by $\beta_{P}$ and $\alpha_{0}^{*}$, are given as follows. $\beta_{P}$ can take any value in the set $\mathcal{A}_{1_{P}}$ (with $\alpha_{0}$ substituted by $\alpha_{0}^{*}$ ), and $\alpha_{0}^{*}=\underset{\alpha_{0}>0}{\operatorname{argmax}} \frac{\frac{P_{a v}}{\tau\left(r, \alpha_{0} z\left(r, \alpha_{0}\right)^{2}\right.}}{\left(1+z\left(r, \alpha_{0}\right)\right)^{2}+P_{a v}}$. This is similar to the RZFBF obtained via a large system analysis based optimization problem for the conventional non-CR MISO-BC system considered in [22] where $\alpha_{0}^{*}=\frac{r}{P_{a v}}$.

Case 2: If $\rho\left(r, \alpha_{0}, \beta\right) \geq \frac{Q_{a v}}{P_{a v}}$ (obviously $\beta \neq 1$ ), in this case, from (13), we have $\bar{P}\left(r, \alpha_{0}, \beta\right)=\frac{Q_{a v}}{\rho\left(r, \alpha_{0}, \beta\right) \tau\left(r, \alpha_{0}\right)}$. Define a set $\mathcal{A}_{2}$ as,

$$
\mathcal{A}_{2} \triangleq\left\{\beta \mid 0 \leq \beta<1, \& \rho\left(r, \alpha_{0}, \beta\right) \geq \frac{Q_{a v}}{P_{a v}}\right\} .
$$

Proposition 4: Based on (22), the set $\mathcal{A}_{2}$ can be rewritten as (23) (shown on the top of the next page).

Proof: See Appendix E.

Then in this case, Problem (18) becomes,

$$
\begin{aligned}
\underset{\beta \in \mathcal{A}_{2}, \alpha_{0}>0}{\operatorname{maximize}} & \bar{P}\left(r, \alpha_{0}, \beta\right) v_{0}\left(r, \alpha_{0}, \beta\right) \\
& =\frac{Q_{a v}}{\tau\left(r, \alpha_{0}\right)} \frac{z\left(r, \alpha_{0}\right)^{2}}{\rho\left(r, \alpha_{0}, \beta\right)\left(1+z\left(r, \alpha_{0}\right)\right)^{2}+Q_{a v}},
\end{aligned}
$$

which is equivalent to the following problem:

$$
\underset{\alpha_{0}>0}{\operatorname{maximize}} g\left(\alpha_{0}\right)
$$

where

$$
g\left(\alpha_{0}\right) \triangleq \underset{\beta \in \mathcal{A}_{2}}{\operatorname{maximize}_{(26)}} \frac{Q_{a v}}{\tau\left(r, \alpha_{0}\right)} \frac{z\left(r, \alpha_{0}\right)^{2}}{\rho\left(r, \alpha_{0}, \beta\right)\left(1+z\left(r, \alpha_{0}\right)\right)^{2}+Q_{a v}}
$$

Proposition 5: The optimal $\beta$ for Problem (26), denoted by $\beta_{Q}$, is given by (27) (shown on the top of the next page). And we always have $\rho\left(r, \alpha_{0}, \beta_{Q}\right)=\frac{Q_{a v}}{P_{a v}}$.

Proof: See Appendix F.

From Proposition 5, $\rho\left(r, \alpha_{0}, \beta_{Q}\right)=\frac{Q_{a v}}{P_{a v}}$ gives $\bar{P}\left(r, \alpha_{0}, \beta_{Q}\right)=\frac{Q_{a v}}{\rho\left(r, \alpha_{0}, \beta_{Q}\right) \tau\left(r, \alpha_{0}\right)}=\frac{P_{a v}}{\tau\left(r, \alpha_{0}\right)}$. Then, $g\left(\alpha_{0}\right)=\frac{\frac{P_{a v}}{\tau\left(r, \alpha_{0}\right)} z\left(r, \alpha_{0}\right)^{2}}{\left(1+z\left(r, \alpha_{0}\right)\right)^{2}+P_{a v}}$. Thus, same as Case 1, Problem (25) also gives $\alpha_{0}^{*}=\frac{r_{a v}}{P_{a v}}$.

Overall, combining the results of Case 1 and Case 2, we can conclude that in the large system limit, the asymptotically optimal regularization parameter $\alpha^{*}$ for the PP-RZFBF scheme is given by $\alpha^{*}=\alpha_{0}^{*} M=\frac{r}{P_{a v}} M=\frac{K}{P_{a v}}$ and the asymptotically optimal projection control parameter $\beta^{*}$ belongs to the set $\mathcal{A}_{3} \triangleq \mathcal{A}_{1} \cup \beta_{Q}$, summarized below as:

- when $0 \leq \sqrt{\frac{Q_{a v}}{P_{a v}}}<1$, we have $\phi_{1}\left(r, \alpha_{0}^{*}\right) \leq \beta^{*} \leq 1$;

- when $1 \leq \sqrt{\frac{Q_{a v}}{P_{a v}}} \leq \frac{1}{2} \frac{1}{\sqrt{\alpha_{0}^{*} z\left(r, \alpha_{0}^{*}\right)\left(1-\alpha_{0}^{*} z\left(r, \alpha_{0}^{*}\right)\right)}}$, we have, if $\alpha_{0}^{*} z\left(r, \alpha_{0}^{*}\right)<0.5$, then $\phi_{1}\left(r, \alpha_{0}^{*}\right) \leq \beta^{*} \leq 1$ or $0 \leq$ $\beta^{*} \leq \phi_{2}\left(r, \alpha_{0}^{*}\right)$; if $\alpha_{0}^{*} z\left(r, \alpha_{0}^{*}\right) \geq 0.5$, then $0 \stackrel{(a)}{\leq} \beta^{*} \leq 1$, where in (a), the equality holds only when $\sqrt{\frac{Q_{a v}}{P_{a v}}}=1$.

- when $\sqrt{\frac{Q_{a v}}{P_{a v}}}>\frac{1}{2} \frac{1}{\sqrt{\alpha_{0}^{*} z\left(r, \alpha_{0}^{*}\right)\left(1-\alpha_{0}^{*} z\left(r, \alpha_{0}^{*}\right)\right)}}$, we have, $0 \leq$ $\beta^{*} \leq 1$.

Remark 8: From the above summarized results, the asymptotically optimal $\alpha_{0}$ (i.e., $\alpha_{0}^{*}=\frac{r}{P_{a v}}$ ) in our CR-MISO-BC system is the same as the conventional non-CR MISO-BC case, and is independent of projection control parameter $\beta$. However the asymptotically optimal $\beta$ (i.e., $\beta^{*}$ ) (or its range of values) is dependent on $\alpha_{0}^{*}$.

Remark 9: Both Case 1 and Case 2 have the same $\bar{P}\left(r, \alpha_{0}^{*}, \beta^{*}\right), v_{0}\left(r, \alpha_{0}^{*}, \beta^{*}\right)$ and the optimal objective function of Problem (18), i.e., $\bar{P}\left(r, \alpha_{0}^{*}, \beta^{*}\right) v_{0}\left(r, \alpha_{0}^{*}, \beta^{*}\right)=z\left(r, \alpha_{0}^{*}\right)$. However, unlike Case 1 where only the ATTP constraint is active, in Case 2, both ATTP and AIP constraints are satisfied with equality. The ATTP constraint is always active in the large system limit, which is consistent with Proposition 1.

Therefore instead of a two-dimensional brute-force search to obtain optimal values of $\alpha$ and $\beta$ for PP-RZFBF scheme, applying $\alpha^{*}$ and the asymptotically optimal range of $\beta^{*}$, we only need to do one-dimensional exhaustive search over set $\mathcal{A}_{3}$ to get the optimal $\beta^{*}$ value, so as to obtain an asymptotically optimal PP-RZFBF beamformer for Problem (7). We call this method as the 'PP-RZFBF-ABF' scheme, which is computationally much less burdensome compared to the optimal PP-RZFBF case.

In the high $P_{a v}$ limit, we can also obtain the following result:

Proposition 6: In the large system limit, for any given $r$, as $P_{a v} \rightarrow \infty$, we have $\alpha_{0}^{*} \rightarrow 0$ and $\beta^{*} \rightarrow 1$. 


$$
\mathcal{A}_{1}= \begin{cases}\left\{\beta \mid \phi_{1}\left(r, \alpha_{0}\right)<\beta \leq 1\right\}, & \text { if } 0 \leq t<1 ; \\ \left\{\beta \mid \phi_{1}\left(r, \alpha_{0}\right)<\beta \leq 1 \| 0 \leq \beta<\phi_{2}\left(r, \alpha_{0}\right)\right\}, & \text { if } 1 \leq t \leq \frac{1}{2} \frac{1}{\sqrt{\alpha_{0} z\left(r, \alpha_{0}\right)\left(1-\alpha_{0} z\left(r, \alpha_{0}\right)\right)}} \& \alpha_{0} z\left(r, \alpha_{0}\right)<0.5 ; \\ \{\beta \mid 0<\beta \leq 1\}, & \text { if } 1 \leq t \leq \frac{1}{2} \frac{1}{\sqrt{\alpha_{0} z\left(r, \alpha_{0}\right)\left(1-\alpha_{0} z\left(r, \alpha_{0}\right)\right)}} \& \alpha_{0} z\left(r, \alpha_{0}\right) \geq 0.5 ; \\ \{\beta \mid 0 \leq \beta \leq 1\}, & \text { otherwise, }\end{cases}
$$

$$
\mathcal{A}_{2}= \begin{cases}\left\{\beta \mid 0 \leq \beta \leq \phi_{1}\left(r, \alpha_{0}\right)\right\}, & \text { if } 0 \leq t<1 ; \\ \{\beta \mid \beta=0\}, & \text { if } t=1 \& \alpha_{0} z\left(r, \alpha_{0}\right) \geq 0.5 ; \\ \left\{\beta \mid \phi_{2}\left(r, \alpha_{0}\right) \leq \beta \leq \phi_{1}\left(r, \alpha_{0}\right)\right\}, & \text { if } 1 \leq t \leq \frac{1}{2} \frac{1}{\sqrt{\alpha_{0} z\left(r, \alpha_{0}\right)\left(1-\alpha_{0} z\left(r, \alpha_{0}\right)\right)}} \& \alpha_{0} z\left(r, \alpha_{0}\right)<0.5 ; \\ \emptyset, & \text { otherwise. }\end{cases}
$$

$$
\beta_{Q}= \begin{cases}\phi_{1}\left(r, \alpha_{0}\right), & \text { if } \quad 0 \leq t<1 \\ 0, & \text { if } \quad t=1 \& \alpha_{0} z\left(r, \alpha_{0}\right) \geq 0.5 \\ \phi_{1}\left(r, \alpha_{0}\right) \text { or } \phi_{2}\left(r, \alpha_{0}\right), & \text { if } \quad 1 \leq t \leq \frac{1}{2} \frac{1}{\sqrt{\alpha_{0} z\left(r, \alpha_{0}\right)\left(1-\alpha_{0} z\left(r, \alpha_{0}\right)\right)}} \& \alpha_{0} z\left(r, \alpha_{0}\right)<0.5 .\end{cases}
$$

Remark 10: From Proposition 6, we can obtain that for the PP-RZFBF scheme, in the large system regime, as $P_{a v} \rightarrow$ $\infty, \tilde{\mathbf{H}}$ asymptotically becomes $\mathbf{H}_{\perp}$, which implies that the secondary channel matrix $\mathbf{H}$ is asymptotically projected into the null space of the primary channel $\mathbf{h}_{0}$. In this case, the interference on PU caused by the secondary transmission is asymptotically removed and thus the AIP constraint becomes asymptotically inactive (see Remark 3 ).

\section{B. Asymptotic Maximum WSR with PP-RZFBF beamforming}

With the values of $\alpha_{0}^{*} \beta^{*}$ obtained above, the maximum asymptotic WSR with PP-RZFBF beamformer in the large system regime for Problem (12) is given as,

$$
\begin{aligned}
& R_{\mathrm{PP}-\mathrm{RZFBF}}^{\infty}{ }^{*}= \\
& \sum_{i=1}^{\bar{K}} w_{i} \log \left(\frac{\bar{K}}{K}+z\left(r, \alpha_{0}^{*}\right)\right)+\sum_{i=1}^{\bar{K}} w_{i} \log \left(\frac{w_{i}}{\frac{1}{K} \sum_{i=1}^{\bar{K}} w_{i}}\right)
\end{aligned}
$$

In the case of $w_{1}=\cdots=w_{K}=w, \bar{K}=K$ and (28) becomes $R_{\mathrm{PP}-\mathrm{RZFBF}}^{\infty}=K w \log \left(1+z\left(r, \alpha_{0}^{*}\right)\right)$.

Remark 11: Obviously, the expression of $R_{\mathrm{PP}-\mathrm{RZFBF}}^{\infty}{ }^{*}$ given above is the same as the asymptotic maximum WSR of the conventional non-CR MISO-BC with RZFBF beamformer (see [35] with the uncorrelated channels case, and for the case of $w_{1}=\cdots=w_{K}=1$, it is also given in [21][22]). Although the obtained $R_{\mathrm{PP}-\mathrm{RZFBF}}{ }^{*}$ for Problem (12) is independent of $\beta^{*}$, it does not mean that the AIP constraint of Problem (12) is always inactive. On the contrary, as we mentioned in the Remark 9, under Case 2, AIP constraint is also satisfied with equality.

In the limit of large $P_{a v}$, we have, $z\left(r, \frac{r}{P_{a v}}\right) \approx$ $\frac{1-r}{r} P_{a v}$, when $r<1 ; z\left(r, \frac{r}{P_{a v}}\right) \approx \sqrt{P_{a v}}$, when $r=1$; and $z\left(r, \frac{r}{P_{a v}}\right) \approx \frac{1}{r-1}$, when $r>1$. Thus the expression for $R_{\mathrm{PP}-\mathrm{RZFBF}}^{\infty}{ }^{*}$ further reduces to (29) (shown on the next page).
Remark 12: Note that from (16), the asymptotically optimal power $p_{i}^{*}=\frac{1}{v_{0}\left(r, \alpha_{0}^{*}, \beta^{*}\right)}\left[\frac{w_{i}\left(\frac{\bar{K}}{K}+z\left(r, \alpha_{0}^{*}\right)\right)}{\frac{1}{K} \sum_{i=1}^{K} w_{i}}-1\right]^{+}, \forall i=$ $1, \ldots, K$. Thus as long as $\frac{1}{K}\left(\frac{\sum_{i=1}^{\bar{K}} w_{i}}{w_{i}}-\bar{K}\right)<z\left(r, \alpha_{0}^{*}\right)$, we will have $p_{i}>0$. As mentioned before, when $r \leq 1$, as $P_{a v} \rightarrow \infty$, we have $z\left(r, \alpha_{0}^{*}\right) \rightarrow \infty$. Therefore in the large $P_{a v}$ regime, given finite $w_{i}, \forall i$, the above inequality is always satisfied, i.e., $p_{i}>0, \forall i=1, \ldots K$ and thus $\bar{K}=K$.

From (29) and Remark 12, we can deduce that for high $P_{a v}$, when $r<1$ (i.e., $K<M$ ), $R_{\mathrm{PP}-\mathrm{RZFBF}}^{\infty}{ }^{*}$ grows linearly with $\sum_{i=1}^{K} w_{i}$. When $r=1$ (i.e., $K=M$ ), the linear growth rate decreases to $\frac{1}{2} \sum_{i=1}^{K} w_{i}$; whereas, when $r>1$ (i.e., $K>$ $M), R_{\mathrm{PP}-\mathrm{RZFBF}}^{\infty}{ }^{*}$ asymptotically approaches a constant. In the specific case of $w_{1}=\cdots=w_{K}=1$, (29) reduces to Equation (24) of [36].

Remark 13: As shown in Section III-B, when $K<M$, for $\beta=1, \alpha=0$, the PP-RZFBF beamforming matrix in (6) reduces to the CZFBF beamforming matrix, given as, $\mathbf{G}=\mathbf{H}_{\perp}\left(\mathbf{H}_{\perp}^{\mathcal{H}} \mathbf{H}_{\perp}\right)^{-1}$, where $\mathbf{H}_{\perp}=\left(\mathbf{I}-\overline{\mathbf{h}}_{0} \overline{\mathbf{h}}_{0}^{\mathcal{H}}\right) \mathbf{H}$. Define $\mathbf{V}_{\perp} \triangleq \mathbf{H}_{\perp}\left(\mathbf{H}_{\perp}^{\mathcal{H}} \mathbf{H}_{\perp}\right)^{-2} \mathbf{H}_{\perp}^{\mathcal{H}}$, then $\mathbf{G}$ can also be written as $\mathbf{G}=\mathbf{V}_{\perp} \mathbf{H}_{\perp}$. Thus, Problem (5) with the suboptimal CZFBF scheme can be expressed as,

$$
\begin{array}{cl}
\underset{\left\{p_{i} \geq 0\right\}_{i=1}^{K}}{\operatorname{maximize}} & R_{\mathrm{CZFBF}}=\sum_{i=1}^{K} E\left[w_{i} \log \left(1+\underline{\operatorname{SINR}}_{i}\right)\right] \\
\text { subject to } & E[\phi] \leq P_{a v}
\end{array}
$$

where $\underline{\operatorname{SINR}}_{i}=\frac{p_{i}\left|\mathbf{h}_{i}^{\mathcal{H}} \mathbf{V}_{\perp} \mathbf{h}_{i}^{\perp}\right|^{2}}{1+\mathbf{h}_{i}^{\mathcal{H}} \mathbf{V}_{\perp} \mathbf{H}_{-i}^{\perp} \mathbf{P}_{-i}\left(\mathbf{H}_{-i}^{\perp}\right)^{\mathcal{H}} \mathbf{V}_{\perp} \mathbf{h}_{i}}$, and $\underline{\phi} \triangleq$ $\sum_{i=1}^{K} p_{i}\left(\mathbf{h}_{i}^{\perp}\right)^{\mathcal{H}}\left(\mathbf{V}_{\perp}\right)^{-2} \mathbf{h}_{i}^{\perp}$ with $\mathbf{h}_{i}^{\perp}$ being the $i^{t h}$ column of $\mathbf{H}_{\perp}$ and $\mathbf{H}_{-i}^{\perp} \triangleq\left\{\mathbf{h}_{1}^{\perp}, \ldots, \mathbf{h}_{i-1}^{\perp}, \mathbf{h}_{i+1}^{\perp}, \ldots, \mathbf{h}_{K}^{\perp}\right\}$. Following a similar analysis used to obtain the asymptotic PP-ZFBF, we can show that in the large system limit, the asymptotic optimal WSR with the CZFBF beamformer, denoted as $R_{\mathrm{CZFBF}}^{\infty}{ }^{*}$, is given as, 


$$
R_{\mathrm{PP}-\mathrm{RZFBF}}^{\infty} * \begin{cases}\sum_{i=1}^{\bar{K}} w_{i} \log \left(P_{a v}\right)+\sum_{i=1}^{\bar{K}} w_{i} \log \left(\frac{w_{i}}{\frac{1}{K} \sum_{i=1}^{K} w_{i}} \frac{1-r}{r}\right), & \text { when } \quad r<1 ; \\ \frac{1}{2} \sum_{i=1}^{\bar{K}} w_{i} \log \left(P_{a v}\right)+\sum_{i=1}^{\bar{K}} w_{i} \log \left(\frac{w_{i}}{\frac{1}{K} \sum_{i=1}^{K} w_{i}}\right), & \text { when } \quad r=1 ; \\ \sum_{i=1}^{\bar{K}} w_{i} \log \left(\left(\frac{\bar{K}}{K}+\frac{1}{r-1}\right) \frac{w_{i}}{\frac{1}{K} \sum_{i=1}^{K} w_{i}}\right)^{\prime}, & \text { when } \quad r>1 .\end{cases}
$$

$$
\begin{aligned}
& R_{\mathrm{CZFBF}}^{\infty}{ }^{*}= \\
& \sum_{i=1}^{\bar{K}} w_{i} \log \left(\frac{\bar{K}}{K}+P_{a v}\left(\frac{1}{r}-1\right)\right)+\sum_{i=1}^{\bar{K}} w_{i} \log \left(\frac{w_{i}}{\frac{1}{K} \sum_{i=1}^{\bar{K}} w_{i}}\right)
\end{aligned}
$$

which is also identical to that of the non-CR MISO-BC with a ZFBF beamformer (see [21] for the case of $w_{1}=\cdots=w_{K}$ ) ${ }^{4}$. Obviously, in the large $P_{a v}$ regime, $R_{\mathrm{CZFBF}}^{\infty}$ has a linear growth rate $\sum_{i=1}^{K} w_{i}$ for $K<M$ similar to $R_{\mathrm{PP}-\mathrm{RZFBF}}^{\infty}$. When $K \geq M$, after user selection, the performance of CZFBF is same as that of the $K<M$ case. Thus the linear scaling rate of $R_{\mathrm{CZFBF}}^{\infty}$ is $\sum_{i \in \mathcal{S}} w_{i}$, which is different to that of PP-RZFBF. More specifically, when $K=M$, the linear scaling rate for asymptotic WSR of PP-RZFBF scheme and CZFBF scheme are $\frac{1}{2} \sum_{i=1}^{K} w_{i}$ and $\sum_{i \in \mathcal{S}} w_{i}$, respectively, which are generally distinct except for the case $K=M=2$ with $w_{1}=w_{2}$. When $K>M$, the asymptotic WSR of PP-RZFBF scheme approaches a constant limiting value and clearly does not have a linear scaling property like the CZFBF scheme.

\section{NumericAl RESUlts}

In this section, we will evaluate the performance of the proposed BF strategies obtained from preceding analysis via numerical simulations. All the channels involved are assumed to undergo Rayleigh fading. All the SUs are assumed to have equal weight, i.e., $w_{1}=\cdots=w_{K}=w=1$, except for Fig. 2(b) (with unequal weight setting).

First, we will test the effectiveness of PP-RZFBF-ABF scheme in the finite system scenario. In Fig. 2, we compare the ergodic WSR performance between the optimized PPRZFBF (obtained via a two-dimensional exhaustive search over all possible $\alpha$ and $\beta$ ), and PP-RZFBF-ABF (with the asymptotically optimal value of $\alpha^{*}$ and range of $\beta^{*}$ obtained from the large system analysis and a one-dimensional exhaustive search for obtaining the optimal $\beta^{*}$ value), in a finite system with $K=M=3$, for $Q_{a v}=0 \mathrm{~dB}$ and $Q_{a v}=10 \mathrm{~dB}$, respectively. As observed from Fig. 2(a) (with equal weight $w_{1}=w_{2}=w_{3}=1$ ), for either value of $Q_{a v}$, the gap between the WSR performance of these two schemes is really small. This indicates that even for small values of $K, M$, the asymptotically optimal $\alpha^{*}$ and $\beta^{*}$ can provide highly accurate approximations. A similar behaviour can be observed in Fig. 2(b) for the unequal weights case with $w_{1}=0.5, w_{2}=1, w_{3}=1.5$. Therefore, in what follows, we

\footnotetext{
${ }^{4}$ Note that both $R_{\mathrm{PP}-\mathrm{RZFBF}}^{\infty}{ }^{*}$ and $R_{\mathrm{CZFBF}}^{\infty}{ }^{*}$ do not depend on $\beta$ and thus their expressions are identical to the corresponding quantities in the non-CR MISO-BC-RZFBF case and non-CR MISO-BC-ZFBF case respectively. By applying $\operatorname{SINR}_{i, z f}^{\infty}=\lim _{\alpha_{0} \rightarrow 0} \operatorname{SINR}_{i, r z f}^{\infty}$ (as obtained in the proof of [21, Theorem 3] for the non-CR MISO-BC case), we can also obtain $R_{\mathrm{CZFBF}}^{\infty}{ }^{*}$ via $R_{\mathrm{CZFBF}}^{\infty}{ }^{*}=\lim _{\alpha_{0} \rightarrow 0} R_{\mathrm{PP}-\mathrm{RZFBF}}^{\infty}{ }^{*}$.
}

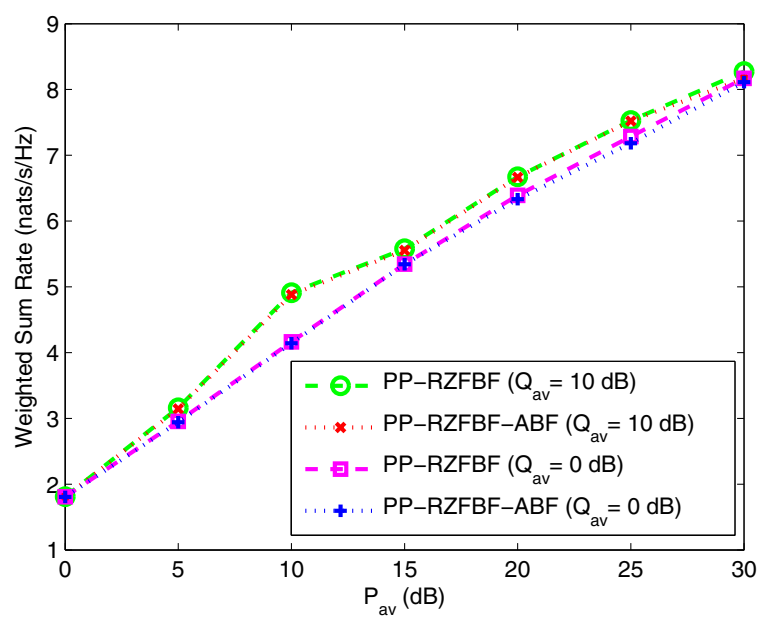

(a) Equal weights $w_{1}=w_{2}=w_{3}=1$

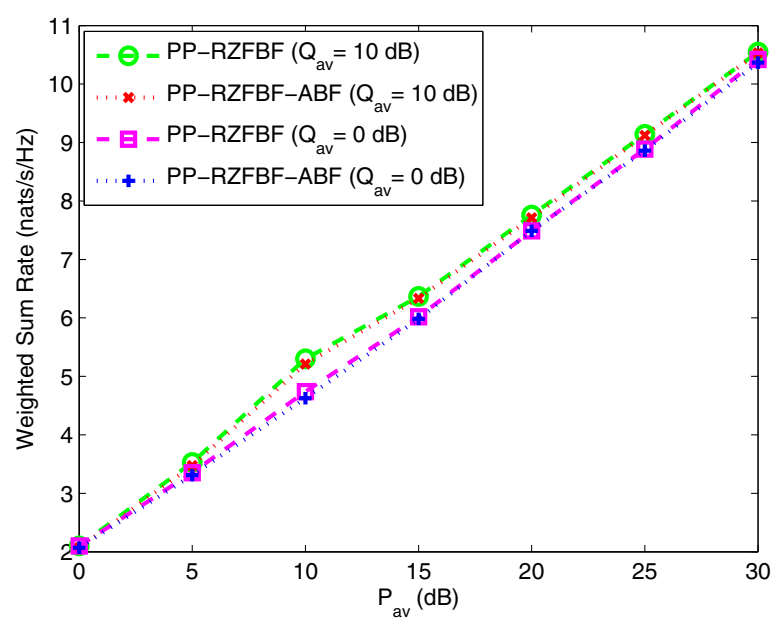

(b) Unequal weights $w_{1}=0.5, w_{2}=1, w_{3}=1.5$

Fig. 2. Ergodic WSR performance comparison between optimal PP-RZFBF and PP-RZFBF with asymptotically optimal beamformer (i.e., PP-RZFBF$\mathrm{ABF}$ ) for $K=M=3$.

will employ PP-RZFBF-ABF to obtain the WSR performance for the PP-RZFBF scheme instead of trying to optimize the PP-RZFBF with a two-dimensional exhaustive search.

Fig. 3 displays the ergodic WSR performance comparison between locally optimum 'BF-LDP-ZGIM' (see [17] for further details) and the two suboptimal algorithms (PP-RZFBF and (ZFBF) versus $P_{a v}$ for $M=4, K=3$, under $Q_{a v}=0$ $\mathrm{dB}$ and $Q_{a v}=10 \mathrm{~dB}$, respectively. First, it can be easily observed that all the WSR curves increase rapidly as $P_{a v}$ increases, which is in agreement with Proposition 1 in that as $P_{a v} \rightarrow \infty$, the optimal WSR obtained by BF-LDP-ZGIM approaches $\infty$ and also Remark 13 in that for large $P_{a v}$, the 


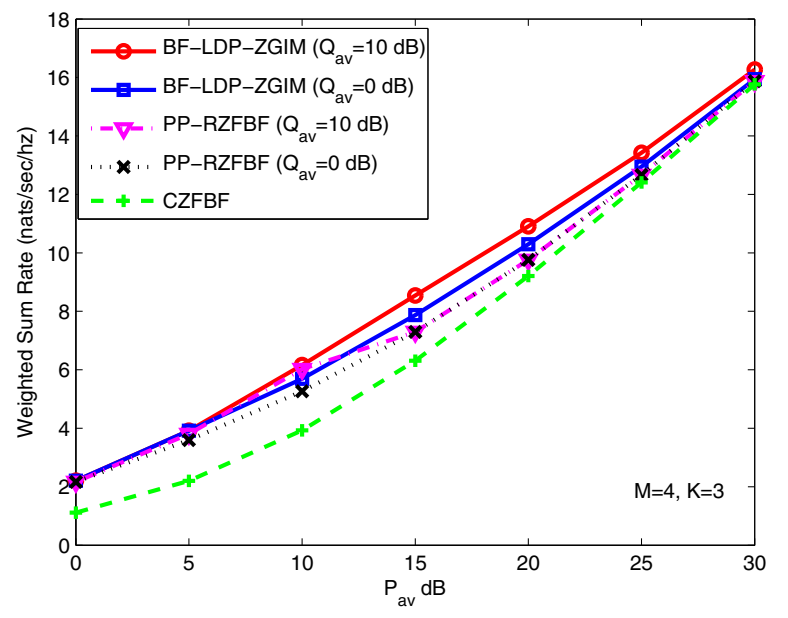

Fig. 3. Ergodic WSR performance comparison between BF-LDP-ZGIM algorithm and two suboptimal algorithms (PP-RZFBF\&CZFBF) for $M=$ $4, K=3$ with different $Q_{a v}$.

asymptotic WSR of PP-RZFBF and CZFBF both have a linear growth rate of $K w$. Fig. 3 also shows that for BF-LDP-ZGIM, higher $Q_{a v}$ always gives better WSR performance as expected. However, for PP-RZFBF, the gain is not so pronounced after $P_{a v} \geq 15 \mathrm{~dB}$. This is because as $P_{a v} \rightarrow \infty, \beta^{*} \rightarrow 1$ (see Proposition 6) for PP-RZFBF. As stated in Remark 10, this essentially implies that the interference caused by the secondary transmission on the PU receiver is asymptotically driven to zero. Thus in high $P_{a v}$, the ergodic WSR performance of PPRZFBF is asymptotically independent of $Q_{a v}$. For the CZFBF scheme, the AIP constraint is always inactive, and thus its ergodic WSR performance does not depend on $Q_{a v}$ at all. Another striking observation from Fig. 3 is that at low $P_{a v}$, the suboptimal PP-RZFBF scheme approaches the optimal BFLDP-ZGIM algorithm, while, as $P_{a v}$ is increased, PP-RZFBF approaches the performance of CZFBF, in agreement with Remark 4 (note that as we mentioned before, due to the high complexity of obtaining the optimal PP-RZFBF, we employ PP-RZFBF-ABF to obtain the PP-RZFBF performance). A similar behaviour can be observed in Fig. 4 for the comparison between PP-RZFBF and CZFBF where $M$ is much bigger than $K(M=10$ and $K=4)$. However, here we observe that PPRZFBF approaches CZFBF much more rapidly in high $P_{a v}$. For example, in Fig.4, with only about $15 \mathrm{~dB}$ of $P_{a v}$, the two schemes are already very close with each other. However in Fig. 3, it requires about $30 \mathrm{~dB}$ of $P_{a v}$ for the two schemes to get close to each other.

Fig. 5 illustrates the ergodic WSR performance of the two suboptimal beamforming algorithms (PP-RZFBF and CZFBF) versus $P_{a v}$ for $M=K=4$ case, under $Q_{a v}=0 \mathrm{~dB}$ and $Q_{a v}=10 \mathrm{~dB}$, respectively. For comparison, the WSR of PPRZFBF with $\beta=1$ and $\alpha \rightarrow 0$ case is also plotted. Similar to Fig. 3, we can also see from Fig. 5 that, when $P_{a v}$ is small, PP-RZFBF with $Q_{a v}=10 \mathrm{~dB}$ significantly outperforms PPRZFBF with $Q_{a v}=0 \mathrm{~dB}$. But after approximately $P_{a v} \geq 15$ $\mathrm{dB}$ the gap between them shrinks and gradually approaches the performance of PP-RZFBF with $\beta=1$ and $\alpha \rightarrow 0$. This confirms the results of Proposition 6 and Remark 10 for

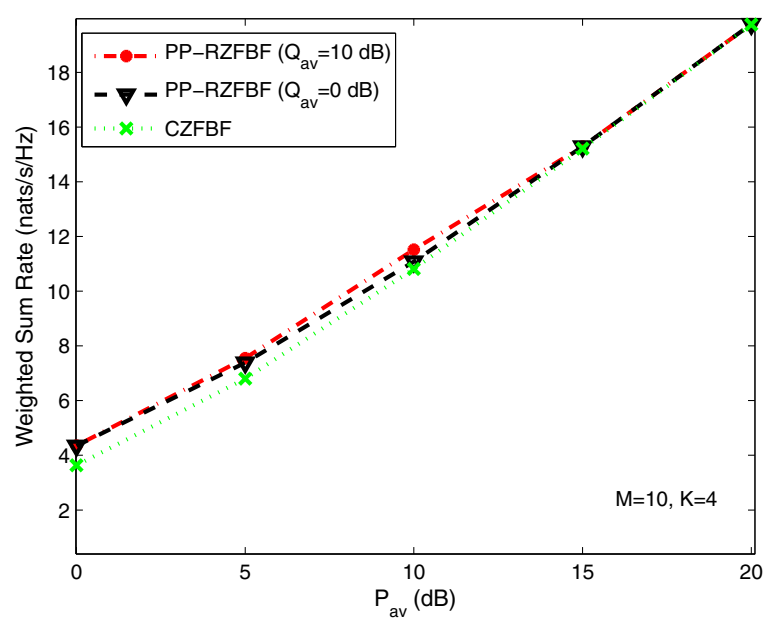

Fig. 4. Ergodic WSR performance comparison between PP-RZFBF scheme and CZFBF scheme for $M=10, K=4$ with different $Q_{a v}$.

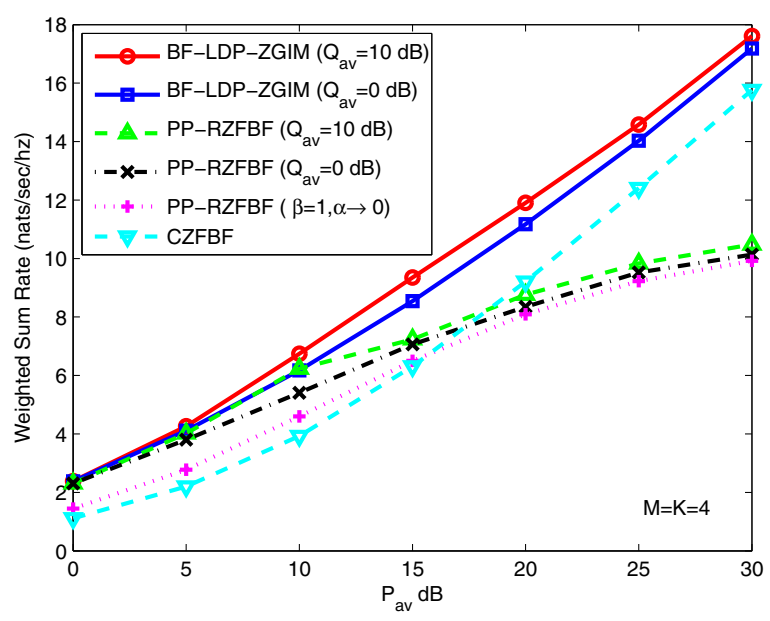

Fig. 5. Ergodic WSR performance comparison between PP-RZFBF, PPRZFBF with $\beta=1$ and $\alpha \rightarrow 0$, and CZFBF for $M=K=4$ with different $Q_{a v}$.

$K=M$ case. However, unlike Fig. 3, in Fig. 5, PP-RZFBF does not always outperform CZFBF, and the curves of PPRZFBF with $\beta=1$ and $\alpha \rightarrow 0$ case and CZFBF are different. This is in agreement with Remark 5 that when $K \geq M$, PPRZFBF does not include CZFBF as a special case.

Fig. 6 further depicts the difference between PP-RZFBF and CZFBF under $Q_{a v}=10 \mathrm{~dB}$ for $M=K=2$ case and $M=K=4$ case, respectively. It can be observed clearly from Fig. 6 that when $M=K=2$, PP-RZFBF outperforms CZFBF and in high $P_{a v}$, both performances grow linearly at a similar rate. On the other hand, when $M=K=4$, PPRZFBF is better than CZFBF at small $P_{a v}$ and they achieve the same WSR at about $P_{a v}=18.5 \mathrm{~dB}$. Past this point, the WSR with CZFBF grows significantly faster than PP-RZFBF. This is due to the fact that (see Remark 13) when $K=M=2$, the asymptotic WSR of PP-RZFBF and CZFBF have an identical linear growth factor $w$, whereas when $K=M=4$, the asymptotic WSR of CZFBF has a larger scaling rate of $3 w$ than that of PP-RZFBF, which is $2 w$. 


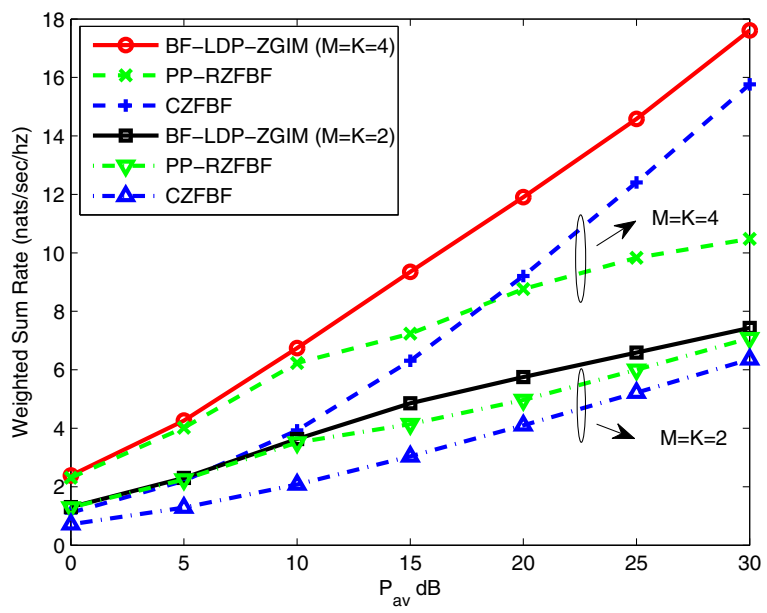

Fig. 6. Ergodic WSR performance comparison between PP-RZFBF and CZFBF with $Q_{a v}=10 \mathrm{~dB}$ for different values of $M=K$.

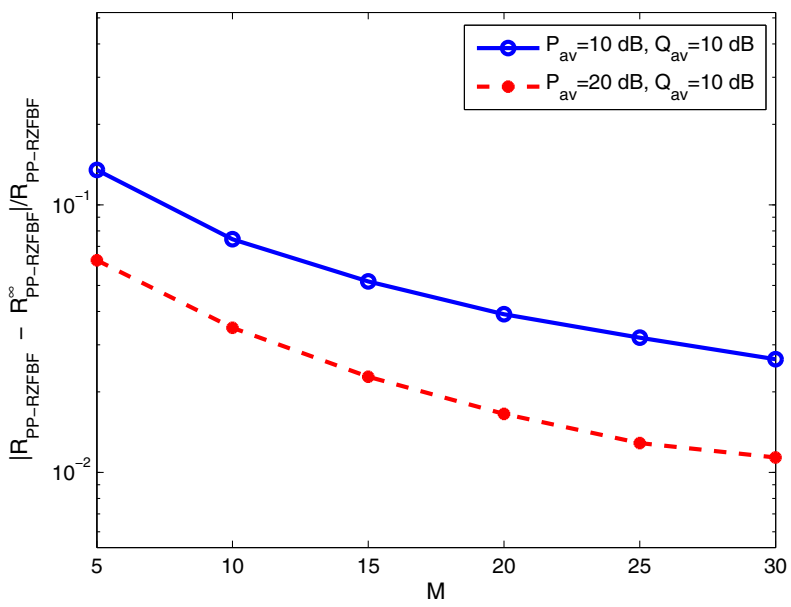

Fig. 7. The asymptotic WSR behaviour of PP-RZFBF for $P_{a v}=10$ and $20 \mathrm{~dB}$ and $Q_{a v}=10 \mathrm{~dB}$ versus $M(=K)$.

Fig. 7 shows the difference between the deterministic WSR approximation of PP-RZFBF, i.e., $R_{\mathrm{PP}-\mathrm{RZFBF}}^{\infty}{ }^{*}$ provided in (28), and $R_{\text {PP-RZFBF }}$ of the corresponding optimal PP-RZFBF scheme for $P_{a v}=10 \& 20 \mathrm{~dB}$ and $Q_{a v}=10 \mathrm{~dB}$ with $M=K$ (taking values from 3 to 30 ). From Fig. 7, we can see that the gap between $R_{\mathrm{PP}-\mathrm{RZFBF}}$ and $R_{\mathrm{PP}-\mathrm{RZFBF}}^{\infty}{ }^{*}$ decreases gradually as $M=K$ increases. This indicates $R_{\mathrm{PP}-\mathrm{RZFBF}}^{\infty}{ }^{*}$ approximates the optimal WSR behaviour extremely well for moderately large values of $M=K$.

\section{DISCUSSION}

In this section we discuss some possible extensions of the current work to more generalized system models. In line with most of the existing works for large system analysis (see, for example, [21][22][23][25][35][37]) dealing with i.i.d. channel models (both gains and noise) for non-CR networks, we also consider i.i.d. channel models in our underlay CR network, so that the existing results for the large system analysis, e.g, Lemma 1 and Lemma 2, can be directly applied. Note that under the i.i.d. channel model assumption, all channels are assumed to undergo identical unity-valued path loss, and same channel noise variance (here assumed to be unity). The extension to non-unity path loss (identical for all channels) and non-unity noise variance are immediate by applying appropriate scaling in the objective function and possibly scaling the maximum allowable average power and interference limits $P_{a v}$ and $Q_{a v}$.

The issue of antenna correlation and non-i.i.d. channels can be also handled, but this requires use of extensions of random matrix theoretic results to non i.i.d. random variables, as presented in [41]. Indeed, such results have been used recently in [21] (see also references therein) to deal with antenna correlation and non-i.i.d. channels (with different path loss etc.) to derive large-system analysis for non-CR (that is, primary/licensed user only) networks. Heterogeneous channel models and antenna correlation are clearly exciting new research directions and will be tackled in future work by suitably adapting the techniques from [21] to our underlay CR network.

Finally, note that for heterogenous (independent but not identically distributed) channels with possibly unequal noise variances, our current results could still be used to derive upper bounds and lower bounds on the weighted sum rate of the CR-MISO-BC network, by considering the corresponding i.i.d. channel models with the best channel conditions and the corresponding i.i.d. channel models with the worst channel conditions, respectively.

Other possible extensions:

Extensions to multiple PUs: Let $L$ denote the number of single-antenna PUs and $\mathbf{h}_{0 n} \in \mathbb{C}^{M \times 1}, n=1, \ldots, L$, represent the channel vector from the CR-BS (secondary base station) to the $n^{t h}$ PU. Problem (5) in this case can be written as,

$\underset{\mathbf{q}_{i}, \forall i=1, \ldots, K}{\operatorname{maximize}} R_{\text {sum }}=\sum_{i=1}^{K} w_{i} E\left[\log \left(1+\frac{\left|\mathbf{h}_{i}^{\mathcal{H}} \mathbf{q}_{i}\right|^{2}}{1+\sum_{j \neq i}^{K}\left|\mathbf{h}_{i}^{\mathcal{H}} \mathbf{q}_{j}\right|^{2}}\right)\right]$ subject to $E\left[\sum_{i=1}^{K}\left|\mathbf{q}_{i}\right|^{2}\right] \leq P_{a v}$,

$$
E\left[\sum_{i=1}^{K}\left|\mathbf{h}_{0 n}^{\mathcal{H}} \mathbf{q}_{i}\right|^{2}\right] \leq Q_{a v}^{n}, \forall n=1, \ldots, L .
$$

where $Q_{a v}^{n}$ is the maximum AIP tolerated by the $\mathrm{PU}_{n}$.

i) If all the CR-BC to primary user channels are i.i.d, the $L$ AIP constraints in Problem (refmpu) reduce to $E\left[\sum_{i=1}^{K}\left|\mathbf{h}_{0}^{\mathcal{H}} \mathbf{q}_{i}\right|^{2}\right] \leq \min \left[Q_{a v}^{1}, \ldots, Q_{a v}^{L}\right]$. Extending our PPRZFBF beamformer idea to this multiple PUs case, a (possibly) suboptimal beamformer for Problem (32) can be given as

$$
\mathbf{G}=\underline{\mathbf{H}}\left(\underline{\mathbf{H}}^{\mathcal{H}} \underline{\mathbf{H}}+\alpha \mathbf{I}\right)^{-1}=\left(\underline{\mathbf{H H}}^{\mathcal{H}}+\alpha \mathbf{I}\right)^{-1} \underline{\mathbf{H}},
$$

where

$$
\underline{\mathbf{H}}=\left(\mathbf{I}-\beta \mathbf{H}_{0 \mathcal{T}}\left(\mathbf{H}_{0 \mathcal{T}}^{\mathcal{H}} \mathbf{H}_{0 \mathcal{T}}\right)^{-1} \mathbf{H}_{0 \mathcal{T}}^{\mathcal{H}}\right) \mathbf{H},
$$

where $\mathbf{H}_{0 \mathcal{T}}=\left\{\mathbf{h}_{0 n}, \forall n \in \mathcal{T}\right\}$ with $\mathcal{T} \subseteq\{1, \ldots, L\}$ being the selected subset of PUs indexes and $1 \leq|\mathcal{T}| \leq L$. (34) implies that, in the multiple PUs case, the secondary channel matrix $\mathbf{H}$ is partially projected into the null space of a selected subset of all the CR-BC to PUs' channels resulting in a general problem 
formulation. How to select the best subset of CR-BC to PUs channels and find the corresponding asymptotically optimal values of $\alpha$ and $\beta$ via large system analysis remain an open question. This is out of the scope of the current paper, but is clearly a very interesting topic for future research. However, when $|\mathcal{T}|=1$, i.e., in (34), $\mathbf{H}$ is partially projected into the null space of only one of CR-BC to PUs' channels as a suboptimal strategy, then the expression (34) becomes similar to the single PU case. In that case, all the results derived in our work for the single PU case can be directly applied.

ii) Extensions to more general heterogeneous channel models (with different noise power, pathloss) and also with antenna correlation need careful examination of the techniques presented in [21] for non-cognitive radio networks and how these can be extended to our underlay cognitive radio setting with multiple PUs. This is again, left for future research.

(iii) Finally, all the above results need to be extended to the more general case of partial/imperfect CSI, especially for the CR-BS to the PUs channels.

\section{CONCLUSiOns}

In this paper, we investigated design of transmit beamforming schemes for an underlay CR-MISO-BC ergodic WSRMax problem, subject to an ATTP constraint at the CRBS and an AIP constraint on the PU. To better analyze the performance of the beamforming schemes, we proposed a low-complexity suboptimal beamforming scheme (called partially-projected regularized zero-forcing beamforming "PPRZFBF") with a close-form beamformer. Large system analysis was then applied to derive deterministic approximations for the PP-RZFBF scheme, based on which, asymptotically optimal value/range of parameters $\beta$ and $\alpha$ can be obtained. We also derived explicit expressions for the asymptotic WSR for PP-RZFBF in the large system regime. A special suboptimal beamforming scheme "CZFBF" was also considered, where all the secondary multiuser interference and the interference on PU are zero-forced. Various comparisons between PPRZFBF and CZFBF were provided. Numerical simulations confirmed the accuracy of asymptomatically optimal $\beta$ and $\alpha$ for PP-RZFBF even in the case of finite-sized systems, and showed that asymptotic expressions of WSR approximate the PP-RZFBF behaviour extremely well for large $M, K$. Future work will extend such beamforming design and associated large system analysis to the various generalizations mentioned in the Discussion Section.

\section{APPENDIX}

\section{A. Proof for Proposition 1}

The proof of the fact that as $P_{a v} \rightarrow \infty, R_{\text {sum }} \rightarrow \infty$ is omitted due to space limit but can be found in the Appendix A of the longer version of this paper in [17].

Let $\lambda^{*}, \mu^{*}, \mathbf{U}_{i}^{*}, \forall i=1, \ldots, K$ be the optimum Lagrange multipliers associated with the ATTP constraint, the AIP constraint and the positive semi-definite (PSD) constraints $\mathbf{S}_{i} \succeq 0, \forall i=1, \ldots, K$ in Problem (4), respectively and we have $\lambda^{*} \geq 0, \mu^{*} \geq 0, \mathbf{U}_{i}^{*} \succeq 0, \forall i=1, \ldots, K$. Let $\left\{\mathbf{S}_{i}^{*}\right\}_{i=1}^{K}$ denote the optimal solution of Problem (4), then $\left\{\mathbf{S}_{i}^{*}\right\}_{i=1}^{K}$ must satisfy the KKT necessary conditions, given as,

$$
\mathbf{U}_{i}^{*}=\mathbf{T}_{-i}-c_{i} \mathbf{h}_{i} \mathbf{h}_{i}^{\mathcal{H}}
$$

where $\mathbf{T}_{-i} \in \mathcal{C}^{M \times M} \triangleq \sum_{j=1, j \neq i}^{K} b_{j} \mathbf{h}_{j} \mathbf{h}_{j}^{\mathcal{H}}+\lambda^{*} \mathbf{I}+\mu^{*} \mathbf{h}_{0} \mathbf{h}_{0}^{\mathcal{H}}$ with $\quad b_{j}=\frac{w_{j} \mathbf{h}_{j}^{\mathcal{H}} \mathbf{S}_{j}^{*} \mathbf{h}_{j}}{\left(1+\sum_{n=1, n \neq j}^{K} \mathbf{h}_{j}^{\mathcal{H}} \mathbf{S}_{n}^{*} \mathbf{h}_{j}\right)\left(1+\sum_{n=1}^{K} \mathbf{h}_{j}^{\mathcal{H}} \mathbf{S}_{n}^{*} \mathbf{h}_{j}\right)}$, and $c_{i}=\frac{w_{i}}{1+\sum_{j=1}^{K} \mathbf{h}_{i}^{\mathcal{H}} \mathbf{S}_{j}^{*} \mathbf{h}_{i}}$. If $\mathbf{T}_{-i}$ is rank-deficient, i.e., $\operatorname{rank}\left(\mathbf{T}_{-i}\right)<M$, we must have $\lambda^{*}=0$ and there must exist a nonzero vector $\mathbf{x} \in \mathcal{C}^{M}$ satisfying $\mathbf{T}_{-i} \mathbf{x}=\mathbf{0}$. Due to the independence of $\mathbf{h}_{i}$ and $\mathbf{h}_{0}, \mathbf{h}_{j}, \forall j \neq i$, we have $\mathbf{x}^{\mathcal{H}} \mathbf{h}_{i} \neq 0$. Then similar to [37, Proposition 1], from (35), we have $\mathbf{x}^{\mathcal{H}} \mathbf{U}_{i}^{*} \mathbf{x}=-c_{i} \mathbf{x}^{\mathcal{H}} \mathbf{h}_{i} \mathbf{h}_{i}^{\mathcal{H}} \mathbf{x}<0$, which violates $\mathbf{U}_{i}^{*} \succeq 0$. Therefore $\mathbf{T}_{-i}$ must have full rank and thus $\operatorname{rank}\left(\mathbf{U}_{i}^{*}\right) \geq M-1$, which gives $\operatorname{rank}\left(\mathbf{S}_{i}^{*}\right) \leq 1$ due to $\mathbf{U}_{i}^{*} \mathbf{S}_{i}^{*}=0$ [2] [37].

Now, we will show that in Problem (4), the ATTP constraint is always active. When $K<M$, we must have $\lambda^{*}>0$, otherwise, $\operatorname{rank}\left(\mathbf{T}_{-i}\right)<M$. Thus in this case, the ATTP constraint is always active. When $K \geq M$, we suppose that in Problem (4), given a pair of values of $P_{a v}$ and $Q_{a v}$, the ATTP constraint is inactive at the optimal solution $\left\{\mathbf{S}_{i}^{*}\right\}_{i=1}^{K}$, i.e., $E\left[\sum_{i=1}^{K} \operatorname{tr}\left\{\mathbf{S}_{i}^{*}\right\}\right]<P_{a v}$, which implies $\lambda^{*}=0$. Then we must have $\mu^{*} \neq 0 .{ }^{5}$ In this case, for every given fading realization $\mathbf{H}_{f} \triangleq\left\{\mathbf{h}_{0}, \mathbf{h}_{1}, \ldots, \mathbf{h}_{K}\right\} \in \mathcal{C}^{M \times(K+1)}$, there must be at least $M$ out of $K$ SUs that are active in order to ensure $\operatorname{rank}\left(\left.\mathbf{T}_{-i}\right|_{\lambda^{*}=0}\right)=M, \forall i=1, \ldots, K$. Let us assume there are $\bar{K}$ active SUs, where $M \leq \bar{K} \leq K$, and without loss of generality, the $\bar{K}$ active SUs are assumed to be $\mathrm{SU}_{1}, \ldots$, $\mathrm{SU}_{\bar{K}}$. Obviously, we have $b_{i} \neq 0, \forall i=1, \ldots, \bar{K}$. We have showed that $\operatorname{rank}\left(\mathbf{U}_{i}^{*}\right) \geq M-1$ and $\mathbf{U}_{i}^{*} \mathbf{S}_{i}^{*}=0, \forall i=1, \ldots, K$, thus if $\operatorname{rank}\left(\mathbf{U}_{i}^{*}\right)=M$, then $\mathbf{S}_{i}^{*}=0$, and if $\mathbf{S}_{i}^{*} \neq 0$, i.e., the user is active, we must have $\operatorname{rank}\left(\mathbf{U}_{i}^{*}\right)=M-1$. Thus we must have $\operatorname{rank}\left(\mathbf{U}_{i}^{*}\right)=M-1$, for $\forall i=1, \ldots, \bar{K}$. In this case, the KKT condition (35) for $i=1, \ldots, \bar{K}$ can be written as, $\mathbf{U}_{i}^{*}=$ $\sum_{j=1, j \neq i}^{K} b_{j} \mathbf{h}_{j} \mathbf{h}_{j}^{\mathcal{H}}+\mu^{*} \mathbf{h}_{0} \mathbf{h}_{0}^{\mathcal{H}}-c_{i} \mathbf{h}_{i} \mathbf{h}_{i}^{\mathcal{H}}=\mathbf{H}_{f} \mathbf{D}_{-i} \mathbf{H}_{f}^{\mathcal{H}}$, where $\mathbf{D}_{-i} \triangleq \operatorname{diag}\left(\mu, b_{1}, \ldots, b_{i-1},-c_{i}, b_{i+1}, \ldots, b_{\bar{K}}, 0, \ldots, 0\right) \in$ $\mathcal{C}^{(K+1) \times(K+1)}$. Obviously, we have $\operatorname{rank}\left(\mathbf{H}_{f}\right)=$ $\operatorname{rank}\left(\mathbf{H}_{f}^{\mathcal{H}}\right)=M$. Let $\mathbf{A}_{i}=\mathbf{H}_{f} \mathbf{D}_{-i}, i=1, \ldots, \bar{K}$, then we have $\mathbf{A}_{i}=\left[\mu \mathbf{h}_{0}, b_{1} \mathbf{h}_{1}, \ldots, b_{i-1} \mathbf{h}_{i-1}\right.$, $\left.-c_{i} \mathbf{h}_{i}, b_{i+1} \mathbf{h}_{i+1}, \ldots, b_{\bar{K}} \mathbf{h}_{\bar{K}}, 0, \ldots, 0\right]$. Due to $\mu \neq 0$, $b_{i} \neq 0, \forall i=1, \ldots, \bar{K}, c_{i} \neq 0$ and $\bar{K} \geq M$, we must have $\operatorname{rank}\left(\mathbf{A}_{i}\right)=M$. Because of $\operatorname{rank}\left(\mathbf{A}_{i} \mathbf{H}_{f}^{\mathcal{H}}\right)=\operatorname{rank}\left(\mathbf{U}_{i}^{*}\right)=M-1$, there must exist a nonzero vector $\mathbf{x} \in \mathcal{C}^{M}$ satisfying $\mathbf{A}_{i} \mathbf{H}_{f}^{\mathcal{H}} \mathbf{x}=\mathbf{0}$. Since $\mathbf{A}_{i}$ is full rank, the vector $\mathbf{H}_{f}^{\mathcal{H}} \mathbf{x}$ must be zero, thus $\mathbf{H}_{f}^{\mathcal{H}}$ must be rank-deficient. However, this violates $\operatorname{rank}\left(\mathbf{H}_{f}^{\mathcal{H}}\right)=M$. Thus with $\lambda^{*}=0, \operatorname{rank}\left(\mathbf{U}_{i}^{*}\right) \neq M-1, \forall i=1, \ldots, \bar{K}$, implying that none of SUs is active, which obviously is not optimal for $K \geq M$. Therefore, we must have $\lambda^{*}>0$, i.e., the ATTP constraint must be always active for $K \geq M$ as well.

\section{B. Proof for Proposition 2}

From the definition of $\Gamma_{-i}$, we can obtain,

${ }^{5}$ Otherwise, both the ATTP and AIP constraints are inactive, then, similar to non-CR case, we can increase the WSR by scaling all the covariance matrices with the same parameter $c>1$, i.e., $\left\{c \mathbf{S}_{i}^{*}\right\}_{i=1}^{K}$ without violating the two constraints, which is in contradiction with the optimality of $\left\{\mathbf{S}_{i}^{*}\right\}_{i=1}^{K}$. 


$$
\begin{aligned}
\Gamma_{-i}^{-1} & =\left(\overline{\mathbf{W}} \hat{\mathbf{H}}_{-i} \hat{\mathbf{H}}_{-i}^{\mathcal{H}} \overline{\mathbf{W}}+\alpha_{0} \mathbf{I}\right)^{-1} \\
& =\left(\hat{\mathbf{H}}_{-i} \hat{\mathbf{H}}_{-i}^{\mathcal{H}}-\beta \frac{M}{\left|\mathbf{h}_{0}\right|^{2}} \hat{\mathbf{H}}_{-i} \hat{\mathbf{H}}_{-i}^{\mathcal{H}} \hat{\mathbf{h}}_{0} \hat{\mathbf{h}}_{0}^{\mathcal{H}}\right. \\
& \left.-\beta \frac{M}{\left|\mathbf{h}_{0}\right|^{2}} \hat{\mathbf{h}}_{0} \hat{\mathbf{h}}_{0}^{\mathcal{H}} \hat{\mathbf{H}}_{-i} \hat{\mathbf{H}}_{-i}^{\mathcal{H}}+\beta^{2}\left(\frac{M}{\left.|| \mathbf{h}_{0}\right|^{2}}\right)^{2} \theta \hat{\mathbf{h}}_{0} \hat{\mathbf{h}}_{0}^{\mathcal{H}}+\alpha_{0} \mathbf{I}\right)^{-1}
\end{aligned}
$$

where $\theta=\hat{\mathbf{h}}_{0}^{\mathcal{H}} \hat{\mathbf{H}}_{-i} \hat{\mathbf{H}}_{-i}^{\mathcal{H}} \hat{\mathbf{h}}_{0}$. Let $\mathbf{C}_{1} \triangleq\left(\hat{\mathbf{H}}_{-i} \hat{\mathbf{H}}_{-i}^{\mathcal{H}}+\alpha_{0} \mathbf{I}\right)$, then since $\left(-\beta \frac{M}{\left|\mathbf{h}_{0}\right|^{2}} \hat{\mathbf{H}}_{-i} \hat{\mathbf{H}}_{-i}^{\mathcal{H}} \hat{\mathbf{h}}_{0} \hat{\mathbf{h}}_{0}^{\mathcal{H}}-\beta \frac{M}{\left|\mathbf{h}_{0}\right|^{2}} \hat{\mathbf{h}}_{0} \hat{\mathbf{h}}_{0}^{\mathcal{H}} \hat{\mathbf{H}}_{-i} \hat{\mathbf{H}}_{-i}^{\mathcal{H}}\right.$ $\left.+\beta^{2}\left(\frac{M}{\left|\mathbf{h}_{0}\right|^{2}}\right)^{2} \theta \hat{\mathbf{h}}_{0} \hat{\mathbf{h}}_{0}^{\mathcal{H}}\right)$ is a rank-one matrix, by applying Lemma 3 (see Appendix H), we have as $M \rightarrow \infty$,

$$
\frac{1}{M} \operatorname{tr}\left\{\Gamma_{-i}^{-1}\right\} \stackrel{a . s .}{\rightarrow} \frac{1}{M} \operatorname{tr}\left\{\mathbf{C}_{1}^{-1}\right\} \stackrel{(a)}{\rightarrow} z\left(r, \alpha_{0}\right)
$$

where (a) is obtained by applying the Lemma 4 (see Appendix $\mathrm{H})$ and $z\left(r, \alpha_{0}\right)=\frac{1}{2}\left[\sqrt{\frac{(1-r)^{2}}{\alpha_{0}{ }^{2}}+\frac{2(1+r)}{\alpha_{0}}+1}+\frac{1-r}{\alpha_{0}}-1\right]$.

Let $\mathbf{C}_{2} \triangleq\left(\mathbf{C}_{1}-\beta \frac{M}{\left|\mathbf{h}_{0}\right|^{2}} \hat{\mathbf{H}}_{-i} \hat{\mathbf{H}}_{-i}^{\mathcal{H}} \hat{\mathbf{h}}_{0} \hat{\mathbf{h}}_{0}^{\mathcal{H}}\right)$ and $\mathbf{C}_{3} \triangleq$ $\left(\mathbf{C}_{2}-\beta \frac{M}{\left|\mathbf{h}_{0}\right|^{2}} \hat{\mathbf{h}}_{0} \hat{\mathbf{h}}_{0}^{\mathcal{H}} \hat{\mathbf{H}}_{-i} \hat{\mathbf{H}}_{-i}^{\mathcal{H}}\right)$, then from (36), we have $\Gamma_{-i}^{-1}=\left(\mathbf{C}_{3}+\beta^{2}\left(\frac{M}{\left|\mathbf{h}_{0}\right|^{2}}\right)^{2} \theta \hat{\mathbf{h}}_{0} \hat{\mathbf{h}}_{0}^{\mathcal{H}}\right)^{-1}$. Applying Matrix Inversion Lemma into $\Gamma_{-i}^{-1}, \mathbf{C}_{3}^{-1}$ and $\mathbf{C}_{2}^{-1}$ respectively, we can get,

$$
\begin{aligned}
& \Gamma_{-i}^{-1}=\mathbf{C}_{3}^{-1}-\frac{\beta^{2}\left(\frac{M}{\left|\mathbf{h}_{0}\right|^{2}}\right)^{2} \theta \mathbf{C}_{3}^{-1} \hat{\mathbf{h}}_{0} \hat{\mathbf{h}}_{0}^{\mathcal{H}} \mathbf{C}_{3}^{-1}}{1+\beta^{2}\left(\frac{M}{\left|\mathbf{h}_{0}\right|^{2}}\right)^{2} \theta \hat{\mathbf{h}}_{0}^{\mathcal{H}} \mathbf{C}_{3}^{-1} \hat{\mathbf{h}}_{0}}, \\
& \mathbf{C}_{3}^{-1}=\mathbf{C}_{2}^{-1}+\frac{\beta \frac{M}{\left|\mathbf{h}_{0}\right|^{2}} \mathbf{C}_{2}^{-1} \hat{\mathbf{h}}_{0} \hat{\mathbf{h}}_{0}^{\mathcal{H}} \hat{\mathbf{H}}_{-i} \hat{\mathbf{H}}_{-i}^{\mathcal{H}} \mathbf{C}_{2}^{-1}}{1-\beta \frac{M}{\left|\mathbf{h}_{0}\right|^{2}} \hat{\mathbf{h}}_{0}^{\mathcal{H}} \hat{\mathbf{H}}_{-i} \hat{\mathbf{H}}_{-i}^{\mathcal{H}} \mathbf{C}_{2}^{-1} \hat{\mathbf{h}}_{0}}, \\
& \mathbf{C}_{2}^{-1}=\mathbf{C}_{1}^{-1}+\frac{\beta \frac{M}{\left|\mathbf{h}_{0}\right|^{2}} \mathbf{C}_{1}^{-1} \hat{\mathbf{H}}_{-i} \hat{\mathbf{H}}_{-i}^{\mathcal{H}} \hat{\mathbf{h}}_{0} \hat{\mathbf{h}}_{0}^{\mathcal{H}} \mathbf{C}_{1}^{-1}}{1-\beta \frac{M}{\left|\mathbf{h}_{0}\right|^{2}} \hat{\mathbf{h}}_{0}^{\mathcal{H}} \mathbf{C}_{1}^{-1} \hat{\mathbf{H}}_{-i} \hat{\mathbf{H}}_{-i}^{\mathcal{H}} \hat{\mathbf{h}}_{0}} .
\end{aligned}
$$

Then, we can easily obtain,

$$
\begin{aligned}
\hat{\mathbf{h}}_{0}^{\mathcal{H}} \Gamma_{-i}^{-1} \hat{\mathbf{h}}_{0} & =\hat{\mathbf{h}}_{0}^{\mathcal{H}} \mathbf{C}_{3}^{-1} \hat{\mathbf{h}}_{0}-\frac{\beta^{2}\left(\frac{M}{\left|\mathbf{h}_{0}\right|^{2}}\right)^{2} \theta\left(\hat{\mathbf{h}}_{0}^{\mathcal{H}} \mathbf{C}_{3}^{-1} \hat{\mathbf{h}}_{0}\right)^{2}}{1+\beta^{2}\left(\frac{M}{\left|\mathbf{h}_{0}\right|^{2}}\right)^{2} \theta \hat{\mathbf{h}}_{0}^{\mathcal{H}} \mathbf{C}_{3}^{-1} \hat{\mathbf{h}}_{0}} \\
& =\frac{\hat{\mathbf{h}}_{0}^{\mathcal{H}} \mathbf{C}_{3}^{-1} \hat{\mathbf{h}}_{0}}{1+\beta^{2}\left(\frac{M}{\left|\mathbf{h}_{0}\right|^{2}}\right)^{2} \theta \hat{\mathbf{h}}_{0}^{\mathcal{H}} \mathbf{C}_{3}^{-1} \hat{\mathbf{h}}_{0}}
\end{aligned}
$$

Similarly, we have,

$$
\hat{\mathbf{h}}_{0}^{\mathcal{H}} \mathbf{C}_{3}^{-1} \hat{\mathbf{h}}_{0}=\frac{\hat{\mathbf{h}}_{0}^{\mathcal{H}} \mathbf{C}_{2}^{-1} \hat{\mathbf{h}}_{0}}{1-\beta \frac{M}{\left|\mathbf{h}_{0}\right|^{2}} \hat{\mathbf{h}}_{0}^{\mathcal{H}} \hat{\mathbf{H}}_{-i} \hat{\mathbf{H}}_{-i}^{\mathcal{H}} \mathbf{C}_{2}^{-1} \hat{\mathbf{h}}_{0}}
$$

and

$$
\begin{gathered}
\hat{\mathbf{h}}_{0}^{\mathcal{H}} \mathbf{C}_{2}^{-1} \hat{\mathbf{h}}_{0}=\frac{\hat{\mathbf{h}}_{0}^{\mathcal{H}} \mathbf{C}_{1}^{-1} \hat{\mathbf{h}}_{0}}{1-\beta \frac{M}{\left|\mathbf{h}_{0}\right|^{2}} \hat{\mathbf{h}}_{0}^{\mathcal{H}} \mathbf{C}_{1}^{-1} \hat{\mathbf{H}}_{-i} \hat{\mathbf{H}}_{-i}^{\mathcal{H}} \hat{\mathbf{h}}_{0}}, \\
\hat{\mathbf{h}}_{0}^{\mathcal{H}} \hat{\mathbf{H}}_{-i} \hat{\mathbf{H}}_{-i}^{\mathcal{H}} \mathbf{C}_{2}^{-1} \hat{\mathbf{h}}_{0}=\hat{\mathbf{h}}_{0}^{\mathcal{H}} \hat{\mathbf{H}}{ }_{-i} \hat{\mathbf{H}}_{-i}^{\mathcal{H}} \mathbf{C}_{1}^{-1} \hat{\mathbf{h}}_{0} \\
+\frac{\beta \frac{M}{\left|\mathbf{h}_{0}\right|^{2}} \hat{\mathbf{h}}_{0}^{\mathcal{H}} \hat{\mathbf{H}}_{-i} \hat{\mathbf{H}}_{-i}^{\mathcal{H}} \mathbf{C}_{1}^{-1} \hat{\mathbf{H}}_{-i} \hat{\mathbf{H}}_{-i}^{\mathcal{H}} \hat{\mathbf{h}}_{{ }^{\prime}} \hat{\mathbf{h}}_{0}^{\mathcal{H}} \mathbf{C}_{1}^{-1} \hat{\mathbf{h}}_{0}}{1-\beta \frac{M}{\left|\mathbf{h}_{0}\right|^{2}} \hat{\mathbf{h}}_{0}^{\mathcal{H}} \mathbf{C}_{1}^{-1} \hat{\mathbf{H}}_{-i} \hat{\mathbf{H}}_{-i}^{\mathcal{H}} \hat{\mathbf{h}}_{0}}
\end{gathered}
$$

From Lemma 1 (see Appendix H), we see that as $M \rightarrow \infty$, $\frac{M}{\left|\mathbf{h}_{0}\right|^{2}}=\frac{1}{\hat{\mathbf{h}}_{0} \hat{\mathbf{h}_{0}}} \stackrel{a . s .}{\rightarrow} \frac{1}{\frac{1}{M} \operatorname{tr}\{\mathbf{I}\}}=1$. Applying this fact and Lemma 1 into (41), we can get, as $M \rightarrow \infty$,

$$
\begin{aligned}
\hat{\mathbf{h}}_{0}^{\mathcal{H}} \mathbf{C}_{2}^{-1} \hat{\mathbf{h}}_{0} \stackrel{a . s .}{\rightarrow} \frac{\frac{1}{M} \operatorname{tr}\left\{\mathbf{C}_{1}^{-1}\right\}}{1-\beta \frac{1}{M} \operatorname{tr}\left\{\mathbf{C}_{1}^{-1} \hat{\mathbf{H}}_{-i} \hat{\mathbf{H}}_{-i}^{\mathcal{H}}\right\}} \\
\hat{\mathbf{h}}_{0}^{\mathcal{H}} \hat{\mathbf{H}}_{-i} \hat{\mathbf{H}}_{-i}^{\mathcal{H}} \mathbf{C}_{2}^{-1} \hat{\mathbf{h}}_{0} \stackrel{a . s .}{\rightarrow} \frac{1}{M} \operatorname{tr}\left\{\hat{\mathbf{H}}_{-i} \hat{\mathbf{H}}_{-i}^{\mathcal{H}} \mathbf{C}_{1}^{-1}\right\} \\
+\frac{\beta \frac{1}{M} \operatorname{tr}\left\{\hat{\mathbf{H}}_{-i} \hat{\mathbf{H}}_{-i}^{\mathcal{H}} \mathbf{C}_{1}^{-1} \hat{\mathbf{H}}_{-i} \hat{\mathbf{H}}_{-i}^{\mathcal{H}}\right\} \frac{1}{M} \operatorname{tr}\left\{\mathbf{C}_{1}^{-1}\right\}}{1-\beta \frac{1}{M} \operatorname{tr}\left\{\mathbf{C}_{1}^{-1} \hat{\mathbf{H}}_{-i} \hat{\mathbf{H}}_{-i}^{\mathcal{H}}\right\}}
\end{aligned}
$$

By applying Lemma 4 (see Appendix H), as $M \rightarrow \infty$, (42) becomes,

$$
\begin{gathered}
\hat{\mathbf{h}}_{0}^{\mathcal{H}} \mathbf{C}_{2}^{-1} \hat{\mathbf{h}}_{0} \stackrel{\text { a.s. }}{\rightarrow} \frac{z\left(r, \alpha_{0}\right)}{1-\beta+\beta \alpha_{0} z\left(r, \alpha_{0}\right)}, \\
\hat{\mathbf{h}}_{0}^{\mathcal{H}} \hat{\mathbf{H}}_{-i} \hat{\mathbf{H}}_{-i}^{\mathcal{H}} \mathbf{C}_{2}^{-1} \hat{\mathbf{h}}_{0} \stackrel{\text { a.s. }}{\rightarrow} 1+\frac{\left(\beta r-\alpha_{0}\right) z\left(r, \alpha_{0}\right)}{1-\beta+\beta \alpha_{0} z\left(r, \alpha_{0}\right)} .
\end{gathered}
$$

Then applying $\frac{M}{\left|\mathbf{h}_{0}\right|^{2}} \stackrel{a . s .}{\rightarrow} 1$ together with (43) into (40), we obtain almost surely, as $M \rightarrow \infty$

$$
\hat{\mathbf{h}}_{0}^{\mathcal{H}} \mathbf{C}_{3}^{-1} \hat{\mathbf{h}}_{0} \stackrel{a . s .}{\rightarrow} \frac{z\left(r, \alpha_{0}\right)}{1+\left(\beta^{2}-2 \beta\right)\left(1-\alpha_{0} z\left(r, \alpha_{0}\right)\right)-\beta^{2} r z\left(r, \alpha_{0}\right)}
$$

Finally, from Lemma 1 and Lemma 4 , we have as $M \rightarrow \infty$, $\theta=\hat{\mathbf{h}}_{0}^{\mathcal{H}} \hat{\mathbf{H}}_{-i} \hat{\mathbf{H}}_{-i}^{\mathcal{H}} \hat{\mathbf{h}}_{0} \stackrel{\text { a.s. }}{\rightarrow} \frac{1}{M} \operatorname{tr}\left\{\hat{\mathbf{H}}_{-i} \hat{\mathbf{H}}_{-i}^{\mathcal{H}}\right\} \stackrel{\text { a.s. }}{\rightarrow} r$. Applying it together with $\frac{M}{\left|\mathbf{h}_{0}\right|^{2}} \stackrel{\text { a.s. }}{\rightarrow} 1$ and (44) into (39), we obtain, as $M \rightarrow \infty$,

$$
\hat{\mathbf{h}}_{0}^{\mathcal{H}} \Gamma_{-i}^{-1} \hat{\mathbf{h}}_{0} \stackrel{a . s .}{\rightarrow} \frac{z\left(r, \alpha_{0}\right)}{1+\left(\beta^{2}-2 \beta\right)\left(1-\alpha_{0} z\left(r, \alpha_{0}\right)\right)}
$$

and (46) (shown on the top of next page), where (a) is obtained by applying $1-\alpha_{0} z\left(r, \alpha_{0}\right)=\frac{z\left(r, \alpha_{0}\right) r}{1+z\left(r, \alpha_{0}\right)}$ into the numerator.

\section{Proof for Theorem 1}

The proof is inspired by the large system analysis in [21][22][23][25] for the non-CR MISO-BC scenario.

(1) Deterministic asymptote for $\operatorname{SINR}_{i}$ :

The $\operatorname{SINR}_{i}$ can be expressed as $\operatorname{SINR}_{i}=\frac{p_{i} U_{1 i}^{2}}{1+U_{2 i}}$, where $U_{1 i} \triangleq \mathbf{h}_{i}^{\mathcal{H}}\left(\tilde{\mathbf{H}} \tilde{\mathbf{H}}^{\mathcal{H}}+\alpha \mathbf{I}\right)^{-1} \tilde{\mathbf{h}}_{i}$ and $U_{2 i} \triangleq \mathbf{h}_{i}^{\mathcal{H}}\left(\tilde{\mathbf{H}} \tilde{\mathbf{H}}^{\mathcal{H}}+\right.$ $\alpha \mathbf{I})^{-1} \tilde{\mathbf{H}}_{-i} \mathbf{P}_{-i} \tilde{\mathbf{H}}_{-i}^{\mathcal{H}}\left(\tilde{\mathbf{H}} \tilde{\mathbf{H}}^{\mathcal{H}}+\alpha \mathbf{I}\right)^{-1} \mathbf{h}_{i}$. First, we will derive the limiting asymptotes for $U_{1 i}$ and $U_{2 i}$, respectively, as $M \rightarrow \infty$ and then apply them back to get $\operatorname{SINR}_{i}^{\infty}$. 


$$
\begin{aligned}
\frac{\partial \hat{\mathbf{h}}_{0}^{\mathcal{H}} \Gamma_{-i}^{-1} \hat{\mathbf{h}}_{0}}{\partial \alpha_{0}} & \stackrel{a . s .}{\rightarrow} \frac{-z\left(r, \alpha_{0}\right)\left(1+z\left(r, \alpha_{0}\right)\right)^{2}\left(1+\left(\beta^{2}-2 \beta\right)\left(1-\alpha_{0} z\left(r, \alpha_{0}\right)\right)\right)+z\left(r, \alpha_{0}\right)^{2} r\left(\beta^{2}-2 \beta\right)}{\left(1+\left(\beta^{2}-2 \beta\right)\left(1-\alpha_{0} z\left(r, \alpha_{0}\right)\right)\right)^{2}\left(r+\alpha_{0}\left(1+z\left(r, \alpha_{0}\right)\right)^{2}\right)} \\
& \stackrel{(a)}{=}-\frac{z\left(r, \alpha_{0}\right)\left(1+z\left(r, \alpha_{0}\right)\right)^{2}+z\left(r, \alpha_{0}\right)^{3} r\left(\beta^{2}-2 \beta\right)}{\left(1+\left(\beta^{2}-2 \beta\right)\left(1-\alpha_{0} z\left(r, \alpha_{0}\right)\right)\right)^{2}\left(r+\alpha_{0}\left(1+z\left(r, \alpha_{0}\right)\right)^{2}\right)}
\end{aligned}
$$

a) Deterministic asymptote for $U_{1 i}$ :

According to the Matrix Inversion Lemma, we can obtain,

$$
\left(\tilde{\mathbf{H}} \tilde{\mathbf{H}}^{\mathcal{H}}+\alpha \mathbf{I}\right)^{-1} \tilde{\mathbf{h}}_{i}=\frac{\left(\tilde{\mathbf{H}}_{-i} \tilde{\mathbf{H}}_{-i}^{\mathcal{H}}+\alpha \mathbf{I}\right)^{-1} \tilde{\mathbf{h}}_{i}}{1+\tilde{\mathbf{h}}_{i}^{\mathcal{H}}\left(\tilde{\mathbf{H}}_{-i} \tilde{\mathbf{H}}_{-i}^{\mathcal{H}}+\alpha \mathbf{I}\right)^{-1} \tilde{\mathbf{h}}_{i}} .
$$

Then with (47) and $\tilde{\mathbf{h}}_{i}=\overline{\mathbf{W}} h_{i}, U_{1 i}$ can be rewritten as, $U_{1 i}=$ $\frac{\hat{\mathbf{h}}_{i}^{\mathcal{H}} \Gamma_{-i}^{-1} \overline{\mathbf{W}} \hat{\mathbf{h}}_{i}}{1+\hat{\mathbf{h}}_{i}^{\mathcal{H}} \overline{\mathbf{W}} \Gamma_{-i}^{-1} \overline{\mathbf{W}} \hat{\mathbf{h}}_{i}}$. By applying Lemma 1, we have, as $M \rightarrow \infty$, $U_{1 i} \stackrel{\text { a.s. }}{\rightarrow} \frac{\frac{1}{M} \operatorname{tr}\left\{\Gamma_{-i}^{-1} \overline{\mathbf{W}}\right\}}{1+\frac{1}{M} \operatorname{tr}\left\{\overline{\mathbf{W}} \Gamma_{-i}^{-1} \overline{\mathbf{W}}\right\}}$. Let $d_{1}^{i} \triangleq \frac{1}{M} \operatorname{tr}\left\{\Gamma_{-i}^{-1} \overline{\mathbf{W}}\right\}$ and $d_{2}^{i} \triangleq$ $\frac{1}{M} \operatorname{tr}\left\{\overline{\mathbf{W}} \Gamma_{-i}^{-1} \frac{\overline{\mathbf{W}}}{\}}\right.$, then applying the definition of $\overline{\mathbf{W}}$, we have

$$
\begin{aligned}
& d_{1}^{i}=\frac{1}{M} \operatorname{tr}\left\{\Gamma_{-i}^{-1}\right\}-\beta \frac{M}{\left|\mathbf{h}_{0}\right|^{2}} \frac{1}{M} \operatorname{tr}\left\{\Gamma_{-i}^{-1} \hat{\mathbf{h}}_{0} \hat{\mathbf{h}}_{0}^{\mathcal{H}}\right\}, \\
& d_{2}^{i}=\frac{1}{M} \operatorname{tr}\left\{\Gamma_{-i}^{-1}\right\}+\left(\beta^{2}-2 \beta\right) \frac{M}{\left|\mathbf{h}_{0}\right|^{2}} \frac{1}{M} \operatorname{tr}\left\{\Gamma_{-i}^{-1} \hat{\mathbf{h}}_{0} \hat{\mathbf{h}}_{0}^{\mathcal{H}}\right\}
\end{aligned}
$$

From Lemma 1, we can obtain as $M \rightarrow \infty, \hat{\mathbf{h}}_{0}^{\mathcal{H}} \hat{\mathbf{h}}_{0} \stackrel{\text { a.s. }}{\rightarrow}$ $\frac{1}{M} \operatorname{tr}\{I\}=1$, thus $\frac{M}{\left|\mathbf{h}_{0}\right|^{2}}=\frac{1}{\hat{\mathbf{h}}_{0}^{\mathcal{H}} \hat{\mathbf{h}}_{0}} \stackrel{\text { a.s. }}{\rightarrow} 1$. Then by applying $\frac{M}{\left|\mathbf{h}_{0}\right|^{2}} \stackrel{a . s .}{\rightarrow} 1$ as $M \rightarrow \infty$ and Proposition 2 into (48), we obtain as $M \rightarrow \infty$, almost surely,

$$
d_{1}^{i} \rightarrow z\left(r, \alpha_{0}\right), \quad d_{2}^{i} \rightarrow z\left(r, \alpha_{0}\right) .
$$

Thus we have, as $M \rightarrow \infty$,

$$
U_{1 i} \stackrel{a . s .}{\rightarrow} \frac{z\left(r, \alpha_{0}\right)}{1+z\left(r, \alpha_{0}\right)}
$$

b) Deterministic asymptote for $U_{2 i}$ :

According to the Matrix Inversion Lemma, we can obtain,

$$
\left(\tilde{\mathbf{H}} \tilde{\mathbf{H}}^{\mathcal{H}}+\alpha \mathbf{I}\right)^{-1}=\frac{1}{M}\left(\Gamma_{-i}^{-1}-\frac{\Gamma_{-i}^{-1} \overline{\mathbf{W}} \hat{\mathbf{h}}_{i} \hat{\mathbf{h}}_{i}^{\mathcal{H}} \overline{\mathbf{W}} \Gamma_{-i}^{-1}}{1+\hat{\mathbf{h}}_{i}^{\mathcal{H}} \overline{\mathbf{W}} \Gamma_{-i}^{-1} \overline{\mathbf{W}} \hat{\mathbf{h}}_{i}}\right) .
$$

With (51), $U_{2 i}$ becomes,

$$
\begin{aligned}
& U_{2 i}=\hat{\mathbf{h}}_{i}^{\mathcal{H}}\left(\Gamma_{-i}^{-1}-\frac{\Gamma_{-i}^{-1} \overline{\mathbf{W}} \hat{\mathbf{h}}_{i} \hat{\mathbf{h}}_{i}^{\mathcal{H}} \overline{\mathbf{W}} \Gamma_{-i}^{-1}}{1+\hat{\mathbf{h}}_{i}^{\mathcal{H}} \overline{\mathbf{W}} \Gamma_{-i}^{-1} \overline{\mathbf{W}} \hat{\mathbf{h}}_{i}}\right) \overline{\mathbf{W}} \hat{\mathbf{H}}_{-i} \mathbf{P}_{-i} \hat{\mathbf{H}}_{-i}^{\mathcal{H}} \overline{\mathbf{W}} \\
& \left(\Gamma_{-i}^{-1}-\frac{\Gamma_{-i}^{-1} \overline{\mathbf{W}} \hat{\mathbf{h}}_{i} \hat{\mathbf{h}}_{i}^{\mathcal{H}} \overline{\mathbf{W}} \Gamma_{-i}^{-1}}{1+\hat{\mathbf{h}}_{i}^{\mathcal{H}} \overline{\mathbf{W}} \Gamma_{-i}^{-1} \overline{\mathbf{W}} \hat{\mathbf{h}}_{i}}\right) \hat{\mathbf{h}}_{i} \\
& =\hat{\mathbf{h}}_{i}^{\mathcal{H}} \Gamma_{-i}^{-1} \overline{\mathbf{W}} \hat{\mathbf{H}}_{-i} \mathbf{P}_{-i} \hat{\mathbf{H}}_{-i}^{\mathcal{H}} \overline{\mathbf{W}} \Gamma_{-i}^{-1} \hat{\mathbf{h}}_{i} \\
& -\frac{\hat{\mathbf{h}}_{i}^{\mathcal{H}} \Gamma_{-i}^{-1} \overline{\mathbf{W}} \hat{\mathbf{H}}_{-i} \mathbf{P}_{-i} \hat{\mathbf{H}}_{-i}^{\mathcal{H}} \overline{\mathbf{W}} \Gamma_{-i}^{-1} \overline{\mathbf{W}} \hat{\mathbf{h}}_{i} \hat{\mathbf{h}}_{i}^{\mathcal{H}} \overline{\mathbf{W}} \Gamma_{-i}^{-1} \hat{\mathbf{h}}_{i}}{1+\hat{\mathbf{h}}_{i}^{\mathcal{H}} \overline{\mathbf{W}} \Gamma_{-i}^{-1} \overline{\mathbf{W}} \hat{\mathbf{h}}_{i}} \\
& -\frac{\hat{\mathbf{h}}_{i}^{\mathcal{H}} \Gamma_{-i}^{-1} \overline{\mathbf{W}} \hat{\mathbf{h}}_{i} \hat{\mathbf{h}}_{i}^{\mathcal{H}} \overline{\mathbf{W}} \Gamma_{-i}^{-1} \overline{\mathbf{W}} \hat{\mathbf{H}}_{-i} \mathbf{P}_{-i} \hat{\mathbf{H}}_{-i}^{\mathcal{H}} \overline{\mathbf{W}} \Gamma_{-i}^{-1} \hat{\mathbf{h}}_{i}}{1+\hat{\mathbf{h}}_{i}^{\mathcal{H}} \overline{\mathbf{W}} \Gamma_{-i}^{-1} \overline{\mathbf{W}} \hat{\mathbf{h}}_{i}} \\
& +\frac{\hat{\mathbf{h}}_{i}^{\mathcal{H}} \Gamma_{-i}^{-1} \overline{\mathbf{W}} \hat{\mathbf{h}}_{i} \hat{\mathbf{h}}_{i}^{\mathcal{H}} \overline{\mathbf{W}} \Gamma_{-i}^{-1} \overline{\mathbf{W}} \hat{\mathbf{H}}_{-i} \mathbf{P}_{-i} \hat{\mathbf{H}}_{-i}^{\mathcal{H}} \overline{\mathbf{W}} \Gamma_{-i}^{-1} \overline{\mathbf{W}} \hat{\mathbf{h}}_{i} \hat{\mathbf{h}}_{i}^{\mathcal{H}} \overline{\mathbf{W}} \Gamma_{-i}^{-1} \hat{\mathbf{h}}_{i}}{\left(1+\hat{\mathbf{h}}_{i}^{\mathcal{H}} \overline{\mathbf{W}} \Gamma_{-i}^{-1} \overline{\mathbf{W}} \hat{\mathbf{h}}_{i}\right)^{2}}
\end{aligned}
$$

After applying Lemma 1 into (52), we have, as $M \rightarrow \infty$,

$$
\begin{aligned}
& U_{2 i} \stackrel{\text { a.s. }}{\rightarrow} \frac{1}{M} \operatorname{tr}\left\{\Gamma_{-i}^{-1} \overline{\mathbf{W}} \hat{\mathbf{H}}_{-i} \mathbf{P}_{-i} \hat{\mathbf{H}}_{-i}^{\mathcal{H}} \overline{\mathbf{W}} \Gamma_{-i}^{-1}\right\} \\
&-2 \frac{\frac{1}{M} \operatorname{tr}\left\{\Gamma_{-i}^{-1} \overline{\mathbf{W}} \hat{\mathbf{H}}_{-i} \mathbf{P}_{-i} \hat{\mathbf{H}}_{-i}^{\mathcal{H}} \overline{\mathbf{W}} \Gamma_{-i}^{-1} \overline{\mathbf{W}}\right\} \frac{1}{M} \operatorname{tr}\left\{\overline{\mathbf{W}} \Gamma_{-i}^{-1}\right\}}{1+\frac{1}{M} \operatorname{tr}\left\{\overline{\mathbf{W}} \Gamma_{-i}^{-1} \overline{\mathbf{W}}\right\}} \\
&+\frac{\left(\frac{1}{M} \operatorname{tr}\left\{\Gamma_{-i}^{-1} \overline{\mathbf{W}}\right\}\right)^{2} \frac{1}{M} \operatorname{tr}\left\{\overline{\mathbf{W}} \Gamma_{-i}^{-1} \overline{\mathbf{W}} \hat{\mathbf{H}}-i \mathbf{P}_{-i} \hat{\mathbf{H}}_{-i}^{\mathcal{H}} \overline{\mathbf{W}} \Gamma_{-i}^{-1} \overline{\mathbf{W}}\right\}}{\left(1+\frac{1}{M} \operatorname{tr}\left\{\overline{\mathbf{W}} \Gamma_{-i}^{-1} \overline{\mathbf{W}}\right\}\right)^{2}} \\
&=d_{3}^{i}-2 \frac{\left(d_{3}^{i}-\beta \frac{M}{\left|\mathbf{h}_{0}\right|^{2}} d_{4}^{i}\right) d_{1}^{i}}{1+d_{2}^{i}}+\frac{\left(d_{1}^{i}\right)^{2}\left(d_{3}^{i}+\left(\beta^{2}-2 \beta\right) \frac{M}{\left|\mathbf{h}_{0}\right|^{2}} d_{4}^{i}\right)}{\left(1+d_{2}^{i}\right)^{2}} .
\end{aligned}
$$

where $d_{3}^{i} \triangleq \frac{1}{M} \operatorname{tr}\left\{\Gamma_{-i}^{-1} \overline{\mathbf{W}} \hat{\mathbf{H}}_{-i} \mathbf{P}_{-i} \hat{\mathbf{H}}_{-i}^{\mathcal{H}} \overline{\mathbf{W}} \Gamma_{-i}^{-1}\right\}, \quad d_{4}^{i} \triangleq$ $\frac{1}{M} \operatorname{tr}\left\{\Gamma_{-i}^{-1} \overline{\mathbf{W}} \hat{\mathbf{H}}_{-i} \mathbf{P}_{-i} \hat{\mathbf{H}}_{-i}^{\mathcal{H}} \overline{\mathbf{W}} \Gamma_{-i}^{-1} \hat{\mathbf{h}}_{0} \hat{\mathbf{h}}_{0}^{\mathcal{H}}\right\}$. Similar to [21], from Lemma 3 (see Appendix $\mathrm{H}$ ), we have, as $M \rightarrow \infty$,

$$
\begin{aligned}
& d_{3}^{i \text { a.s. }} \rightarrow \hat{d}_{3}^{i} \triangleq \frac{1}{M} \operatorname{tr}\left\{\Gamma^{-1} \overline{\mathbf{W}} \hat{\mathbf{H}}_{-i} \mathbf{P}_{-i} \hat{\mathbf{H}}_{-i}^{\mathcal{H}} \overline{\mathbf{W}} \Gamma^{-1}\right\} \\
& d_{4}^{i \text { a.s. }} \rightarrow \hat{d}_{4}^{i} \triangleq \frac{1}{M} \operatorname{tr}\left\{\Gamma^{-1} \overline{\mathbf{W}} \hat{\mathbf{H}}_{-i} \mathbf{P}_{-i} \hat{\mathbf{H}}_{-i}^{\mathcal{H}} \overline{\mathbf{W}} \Gamma^{-1} \hat{\mathbf{h}}_{0} \hat{\mathbf{h}}_{0}^{\mathcal{H}}\right\}
\end{aligned}
$$

where $\Gamma \triangleq\left(\frac{\tilde{\mathbf{H}} \tilde{\mathbf{H}}^{\mathcal{H}}}{M}+\alpha_{0} \mathbf{I}\right) \cdot \hat{d}_{3}^{i}$ can be rewritten as,

$$
\begin{aligned}
\hat{d}_{3}^{i} & =\frac{1}{M} \operatorname{tr}\left\{\mathbf{P}_{-i} \hat{\mathbf{H}}_{-i}^{\mathcal{H}} \overline{\mathbf{W}} \Gamma^{-2} \overline{\mathbf{W}} \hat{\mathbf{H}}_{-i}\right\} \\
& =\frac{1}{M} \sum_{j=1, j \neq i}^{K} p_{j} \hat{\mathbf{h}}_{j}^{\mathcal{H}} \overline{\mathbf{W}} \Gamma^{-2} \overline{\mathbf{W}} \hat{\mathbf{h}}_{j} \\
& \stackrel{(a)}{=} \frac{1}{M} \sum_{j=1, j \neq i}^{K} p_{j} \frac{\hat{\mathbf{h}}_{j}^{\mathcal{H}} \overline{\mathbf{W}} \Gamma_{-j}^{-2} \overline{\mathbf{W}} \hat{\mathbf{h}}_{j}}{\left(1+\hat{\mathbf{h}}_{j}^{\mathcal{H}} \overline{\mathbf{W}} \Gamma_{-j}^{-1} \overline{\mathbf{W}} \hat{\mathbf{h}}_{j}\right)^{2}}
\end{aligned}
$$

where (a) is obtained by applying matrix inversion lemma twice with $\Gamma_{-j}=\left(\overline{\mathbf{W}} \hat{\mathbf{H}}_{-j} \hat{\mathbf{H}}_{-j}^{\mathcal{H}} \overline{\mathbf{W}}+\alpha_{0} \mathbf{I}\right)$, and $\hat{\mathbf{H}}_{-j}$ is the matrix of $\hat{\mathbf{H}}$ where the $j^{\text {th }}$ columns are removed. Applying Lemma 1 , we get as $M \rightarrow \infty$,

$$
\begin{aligned}
& \hat{d}_{3}^{i} \stackrel{a . s .}{\rightarrow} \frac{1}{M} \sum_{j=1, j \neq i}^{K} p_{j} \frac{\frac{1}{M} \operatorname{tr}\left\{\overline{\mathbf{W}} \Gamma_{-j}^{-2} \overline{\mathbf{W}}\right\}}{\left(1+\frac{1}{M} \operatorname{tr}\left\{\overline{\mathbf{W}} \Gamma_{-j}^{-1} \overline{\mathbf{W}}\right\}\right)^{2}} \\
& \stackrel{(a)}{=} r \frac{1}{K} \sum_{j=1, j \neq i}^{K} p_{j} \frac{-\frac{\partial \frac{1}{M} \operatorname{tr}\left\{\overline{\mathbf{W}} \Gamma_{-j}^{-1} \overline{\mathbf{W}}\right\}}{\partial \alpha_{0}}}{\left(1+\frac{1}{M} \operatorname{tr}\left\{\overline{\mathbf{W}} \Gamma_{-j}^{-1} \overline{\mathbf{W}}\right\}\right)^{2}} \\
& \stackrel{(b)}{a . s .} \frac{r z\left(r, \alpha_{0}\right)}{r+\alpha_{0}\left(1+z\left(r, \alpha_{0}\right)\right)^{2}}\left(\frac{1}{K} \sum_{j=1, j \neq i}^{K} p_{j}\right)
\end{aligned}
$$

where $(a)$ is obtained due to $-\frac{\partial \operatorname{tr}\left\{\Gamma_{-j}^{-1} \overline{\mathbf{W}}^{2}\right\}}{\partial \alpha_{0}}=-\operatorname{tr}\left\{\frac{\partial \Gamma_{-j}^{-1}}{\partial \alpha_{0}} \overline{\mathbf{W}}^{2}\right\}=$ $\operatorname{tr}\left\{\Gamma_{-j}^{-1} \frac{\partial \Gamma_{-j}}{\partial \alpha_{0}} \Gamma_{-j}^{-1} \overline{\mathbf{W}}^{2}\right\}=\operatorname{tr}\left\{\Gamma_{-j}^{-2} \overline{\mathbf{W}}^{2}\right\}$, and in (b), $\frac{1}{M} \operatorname{tr}\left\{\overline{\mathbf{W}} \Gamma_{-j}^{-1} \overline{\mathbf{W}}\right\} \stackrel{\text { a.s. }}{\rightarrow} z\left(r, \alpha_{0}\right)$, i.e., $d_{2}^{i}$ in (49), is applied. 
Similarly, $\hat{d}_{4}^{i}$ also can be expressed as,

$$
\begin{aligned}
\hat{d}_{4}^{i} & =\frac{1}{M} \operatorname{tr}\left\{\mathbf{P}_{-i} \hat{\mathbf{H}}_{-i}^{\mathcal{H}} \overline{\mathbf{W}} \Gamma^{-1} \hat{\mathbf{h}}_{0} \hat{\mathbf{h}}_{0}^{\mathcal{H}} \Gamma^{-1} \overline{\mathbf{W}} \hat{\mathbf{H}}_{-i}\right\} \\
& =\frac{1}{M} \sum_{j=1, j \neq i}^{K} p_{j} \hat{\mathbf{h}}_{j}^{\mathcal{H}} \overline{\mathbf{W}} \Gamma^{-1} \hat{\mathbf{h}}_{0} \hat{\mathbf{h}}_{0}^{\mathcal{H}} \Gamma^{-1} \overline{\mathbf{W}} \hat{\mathbf{h}}_{j} \\
& =\frac{1}{M} \sum_{j=1, j \neq i}^{K} p_{j} \frac{\hat{\mathbf{h}}_{j}^{\mathcal{H}} \overline{\mathbf{W}} \Gamma_{-j}^{-1} \hat{\mathbf{h}}_{0} \hat{\mathbf{h}}_{0}^{\mathcal{H}} \Gamma_{-j}^{-1} \overline{\mathbf{W}} \hat{\mathbf{h}}_{j}}{\left(1+\hat{\mathbf{h}}_{j}^{\mathcal{H}} \overline{\mathbf{W}} \Gamma_{-j}^{-1} \overline{\mathbf{W}} \hat{\mathbf{h}}_{j}\right)^{2}} \\
& \stackrel{a . s .}{\rightarrow} \frac{1}{M} \sum_{j=1, j \neq i}^{K} p_{j} \frac{\frac{1}{M} \operatorname{tr}\left\{\overline{\mathbf{W}} \Gamma_{-j}^{-1} \hat{\mathbf{h}}_{0} \hat{\mathbf{h}}_{0}^{\mathcal{H}} \Gamma_{-j}^{-1} \overline{\mathbf{W}}\right\}}{\left(1+\frac{1}{M} \operatorname{tr}\left\{\overline{\mathbf{W}} \Gamma_{-j}^{-1} \overline{\mathbf{W}}\right\}\right)^{2}}
\end{aligned}
$$

By applying Proposition 2, we can get (58) (shown on the top of the next page), where the last equality is obtained by applying $z r=(1+z)\left(1-\alpha_{0} z\right)$. Applying (58) into (57), we have, as $M \rightarrow \infty$,

$$
\begin{aligned}
& \hat{d}_{4}^{i a . s .} \\
& \frac{1}{M} \frac{r(1-\beta)^{2} z\left(r, \alpha_{0}\right)\left(\frac{1}{K} \sum_{j=1, j \neq i}^{K} p_{j}\right)}{\left(1+\left(\beta^{2}-2 \beta\right)\left(1-\alpha_{0} z\left(r, \alpha_{0}\right)\right)\right)^{2}\left(r+\alpha_{0}\left(1+z\left(r, \alpha_{0}\right)\right)^{2}\right)} .
\end{aligned}
$$

Substituting (49), (56) and (59) into (53), yields, as $M \rightarrow \infty$,

$$
U_{2 i} \stackrel{a . s .}{\rightarrow} \frac{r z\left(r, \alpha_{0}\right)\left(\frac{1}{K} \sum_{j=1, j \neq i}^{K} p_{j}\right)}{\left(r+\alpha_{0}\left(1+z\left(r, \alpha_{0}\right)\right)^{2}\right)\left(1+z\left(r, \alpha_{0}\right)\right)^{2}}
$$

Therefore, with (50) and (60), as $M \rightarrow \infty$, we can obtain (61) (shown on the top of the next page).

(2) Deterministic asymptote for $\phi$ :

By using (47), $\phi$ becomes

$$
\begin{aligned}
\phi & =\sum_{i=1}^{K} p_{i} \frac{\frac{1}{M} \hat{\mathbf{h}}_{i}^{\mathcal{H}} \overline{\mathbf{W}} \Gamma_{-i}^{-2} \overline{\mathbf{W}} \hat{\mathbf{h}}_{i}}{\left(1+\hat{\mathbf{h}}_{i}^{\mathcal{H}} \overline{\mathbf{W}} \Gamma_{-i}^{-1} \overline{\mathbf{W}} \hat{\mathbf{h}}_{i}\right)^{2}} \\
& \stackrel{\text { a.s. }}{\rightarrow} \sum_{i=1}^{K} p_{i} \frac{\frac{1}{M^{2}} \operatorname{tr}\left\{\overline{\mathbf{W}} \Gamma_{-i}^{-2} \overline{\mathbf{W}}\right\}}{\left(1+\frac{1}{M} \operatorname{tr}\left\{\overline{\mathbf{W}} \Gamma_{-i}^{-1} \overline{\mathbf{W}}\right\}\right)^{2}} \\
& =r \frac{-\frac{\partial d_{2}^{i}}{\partial \alpha_{0}}}{\left(1+d_{2}^{i}\right)^{2}}\left(\frac{1}{K} \sum_{i=1}^{K} p_{i}\right) \\
& \stackrel{r . s .}{\rightarrow} \frac{r z\left(r, \alpha_{0}\right)}{r+\alpha_{0}\left(1+z\left(r, \alpha_{0}\right)\right)^{2}}\left(\frac{1}{K} \sum_{i=1}^{K} p_{i}\right)
\end{aligned}
$$

(3) Deterministic asymptote for $\psi$ :

By using (47), $\psi$ can be written as,

$$
\begin{gathered}
\psi=\sum_{i=1}^{K} p_{i} \frac{\hat{\mathbf{h}}_{i}^{\mathcal{H}} \overline{\mathbf{W}} \Gamma_{-i}^{-1} \hat{\mathbf{h}}_{0} \hat{\mathbf{h}}_{0}^{\mathcal{H}} \Gamma_{-i}^{-1} \overline{\mathbf{W}} \hat{\mathbf{h}}_{i}}{\left(1+\hat{\mathbf{h}}_{i}^{\mathcal{H}} \overline{\mathbf{W}} \Gamma_{-i}^{-1} \overline{\mathbf{W}} \hat{\mathbf{h}}_{i}\right)^{2}} \\
\stackrel{\text { a.s. }}{\rightarrow} \sum_{i=1}^{K} p_{i} \frac{\frac{1}{M} \operatorname{tr}\left\{\overline{\mathbf{W}} \Gamma_{-i}^{-1} \hat{\mathbf{h}}_{0} \hat{\mathbf{h}}_{0}^{\mathcal{H}} \Gamma_{-i}^{-1} \overline{\mathbf{W}}\right\}}{\left(1+d_{2}^{i}\right)^{2}}
\end{gathered}
$$

By applying (58), we have

$$
\psi \stackrel{a . s .}{\rightarrow} \frac{r(1-\beta)^{2} z\left(r, \alpha_{0}\right)\left(\frac{1}{K} \sum_{i=1}^{K} p_{i}\right)}{\left(1+\left(\beta^{2}-2 \beta\right)\left(1-\alpha_{0} z\left(r, \alpha_{0}\right)\right)\right)^{2}\left(r+\alpha_{0}\left(1+z\left(r, \alpha_{0}\right)\right)^{2}\right)} \text {. }
$$

\section{Proof for Proposition 3}

Let $t=\sqrt{\frac{Q_{a v}}{P_{a v}}}$. Applying the definition of $\rho\left(r, \alpha_{0}, \beta\right)$ into $\rho\left(r, \alpha_{0}, \beta\right)<\frac{Q_{a v}}{P_{a v}}$, gives,

$$
\begin{aligned}
& \left(\beta+\frac{1}{2 t\left(1-\alpha_{0} z\left(r, \alpha_{0}\right)\right)}-1\right)^{2} \\
& >1-\frac{1}{1-\alpha_{0} z\left(r, \alpha_{0}\right)}+\frac{1}{4 t^{2}\left(1-\alpha_{0} z\left(r, \alpha_{0}\right)\right)^{2}}
\end{aligned}
$$

1) when $t>\frac{1}{2} \frac{1}{\sqrt{\alpha_{0} z\left(r, \alpha_{0}\right)\left(1-\alpha_{0} z\left(r, \alpha_{0}\right)\right)}}$ (implying $1-$ $\left.\frac{1}{1-\alpha_{0} z\left(r, \alpha_{0}\right)}+\frac{1}{4 t^{2}\left(1-\alpha_{0} z\left(r, \alpha_{0}\right)\right)^{2}}<0\right)$, in this case, (19) becomes $0 \leq \beta \leq 1$.

2) when $t \leq \frac{1}{2} \frac{1}{\sqrt{\alpha_{0} z\left(r, \alpha_{0}\right)\left(1-\alpha_{0} z\left(r, \alpha_{0}\right)\right)}}$, in this case, (65) gives $\beta>\phi_{1}\left(r, \alpha_{0}\right)$ or $\beta<\phi_{2}\left(r, \alpha_{0}\right)$, where the definitions of $\phi_{1}\left(r, \alpha_{0}\right)$ and $\phi_{2}\left(r, \alpha_{0}\right)$ are given by (21). Obviously, $\phi_{2}\left(r, \alpha_{0}\right)<\phi_{1}\left(r, \alpha_{0}\right)$ and $\phi_{1}\left(r, \alpha_{0}\right)<$ $\left(\sqrt{\frac{1}{4 t^{2}\left(1-\alpha_{0} z\left(r, \alpha_{0}\right)\right)^{2}}}-\frac{1}{2 t\left(1-\alpha_{0} z\left(r, \alpha_{0}\right)\right)}+1\right)=1$. Then $(19)$ becomes,

$\max \left\{0, \phi_{1}\left(r, \alpha_{0}\right)\right\}<\beta \leq 1$ or $0 \leq \beta<\max \left\{0, \phi_{2}\left(r, \alpha_{0}\right)\right\}$.

Note that $\quad \frac{1}{2} \frac{1}{\sqrt{\alpha_{0} z\left(r, \alpha_{0}\right)\left(1-\alpha_{0} z\left(r, \alpha_{0}\right)\right)}}$ = $\frac{1}{2} \frac{1}{\sqrt{0.25-\left(0.5-\alpha_{0} z\left(r, \alpha_{0}\right)\right)^{2}}} \geq 1$. Then from (21), it is easy to get that,

(i) when $t \in\left(\frac{1}{2\left(1-\alpha_{0} z\left(r, \alpha_{0}\right)\right)}, \frac{1}{2} \frac{1}{\sqrt{\alpha_{0} z\left(r, \alpha_{0}\right)\left(1-\alpha_{0} z\left(r, \alpha_{0}\right)\right)}}\right]$, or $t \in\left[0, \frac{1}{2\left(1-\alpha_{0} z\left(r, \alpha_{0}\right)\right)}\right] \cap[0,1)$, we have $\max \left\{0, \phi_{1}\left(r, \alpha_{0}\right)\right\}=\phi_{1}\left(r, \alpha_{0}\right)$; while, when $1 \leq$ $t \leq \min \left[\frac{1}{2\left(1-\alpha_{0} z\left(r, \alpha_{0}\right)\right)}, \frac{1}{2} \frac{1}{\sqrt{\alpha_{0} z\left(r, \alpha_{0}\right)\left(1-\alpha_{0} z\left(r, \alpha_{0}\right)\right)}}\right]$, we have $\max \left\{0, \phi_{1}\left(r, \alpha_{0}\right)\right\}=0$.

(ii) when $\max \left[\frac{1}{2\left(1-\alpha_{0} z\left(r, \alpha_{0}\right)\right)}, 1\right]<t \leq$ $\frac{1}{2} \frac{1}{\sqrt{\alpha_{0} z\left(r, \alpha_{0}\right)\left(1-\alpha_{0} z\left(r, \alpha_{0}\right)\right)}}, \quad$ we have $\max \left\{0, \phi_{2}\left(r, \alpha_{0}\right)\right\}=\phi_{2}\left(r, \alpha_{0}\right) ; \quad$ Otherwise, $\max \left\{0, \phi_{2}\left(r, \alpha_{0}\right)\right\}=0$.

Based on the above (i) and (ii), we can obtain,

2.1) If $\alpha_{0} z\left(r, \alpha_{0}\right)<0.5$, then we have $\frac{1}{2\left(1-\alpha_{0} z\left(r, \alpha_{0}\right)\right)}<1$ and $\frac{1}{2\left(1-\alpha_{0} z\left(r, \alpha_{0}\right)\right)}<\frac{1}{2} \frac{1}{\sqrt{\alpha_{0} z\left(r, \alpha_{0}\right)\left(1-\alpha_{0} z\left(r, \alpha_{0}\right)\right)}}$. In this case, according to (i) and (ii), we have when $0 \leq t \leq \frac{1}{2} \frac{1}{\sqrt{\alpha_{0} z\left(r, \alpha_{0}\right)\left(1-\alpha_{0} z\left(r, \alpha_{0}\right)\right)}}, \max \left\{0, \phi_{1}\left(r, \alpha_{0}\right)\right\}$ $=\phi_{1}\left(r, \alpha_{0}\right)$; when $1<t \leq \frac{1}{2} \frac{1}{\sqrt{\alpha_{0} z\left(r, \alpha_{0}\right)\left(1-\alpha_{0} z\left(r, \alpha_{0}\right)\right)}}$, $\max \left\{0, \phi_{2}\left(r, \alpha_{0}\right)\right\}=\phi_{2}\left(r, \alpha_{0}\right)$, otherwise, $\max \left\{0, \phi_{2}\left(r, \alpha_{0}\right)\right\}=0$. Thus (66) becomes,

$\left\{\begin{array}{r}\phi_{1}\left(r, \alpha_{0}\right)<\beta \leq 1 \quad \text { when } 0 \leq t \leq 1 ; \\ \phi_{1}\left(r, \alpha_{0}\right)<\beta \leq 1, \quad \text { or } 0 \leq \beta<\phi_{2}\left(r, \alpha_{0}\right), \\ \text { when } 1<t \leq \frac{1}{2} \frac{1}{\sqrt{\alpha_{0} z\left(r, \alpha_{0}\right)\left(1-\alpha_{0} z\left(r, \alpha_{0}\right)\right)}} .\end{array}\right.$

(67) can be rewritten as,

$\left\{\begin{array}{r}\phi_{1}\left(r, \alpha_{0}\right)<\beta \leq 1 \\ \phi_{1}\left(r, \alpha_{0}\right)<\beta \leq 1, \quad \text { or } 0 \leq \beta<\phi_{2}\left(r, \alpha_{0}\right), \\ \text { when } 1 \leq t \leq \frac{1}{2} \frac{1}{\sqrt{\alpha_{0} z\left(r, \alpha_{0}\right)\left(1-\alpha_{0} z\left(r, \alpha_{0}\right)\right)}} .\end{array}\right.$ 


$$
\begin{aligned}
\hat{\mathbf{h}}_{0}^{\mathcal{H}} \Gamma_{-i}^{-1} \overline{\mathbf{W W}} \Gamma_{-i}^{-1} \hat{\mathbf{h}}_{0} & =\hat{\mathbf{h}}_{0}^{\mathcal{H}} \Gamma_{-i}^{-2} \hat{\mathbf{h}}_{0}+\left(\beta^{2}-2 \beta\right) \frac{M}{\left|\mathbf{h}_{0}\right|^{2}}\left(\hat{\mathbf{h}}_{0}^{\mathcal{H}} \Gamma_{-i}^{-1} \hat{\mathbf{h}}_{0}\right)^{2}=-\frac{\partial \hat{\mathbf{h}}_{0}^{\mathcal{H}} \Gamma_{-i}^{-1} \hat{\mathbf{h}}_{0}}{\partial \alpha_{0}}+\left(\beta^{2}-2 \beta\right) \frac{M}{\left|\mathbf{h}_{0}\right|^{2}}\left(\hat{\mathbf{h}}_{0}^{\mathcal{H}} \Gamma_{-i}^{-1} \hat{\mathbf{h}}_{0}\right)^{2} \\
& \stackrel{a . s .}{\rightarrow} \frac{z\left(r, \alpha_{0}\right)\left(1+z\left(r, \alpha_{0}\right)\right)^{2}+\left(\beta^{2}-2 \beta\right) z^{2}\left(r, \alpha_{0}\right)\left(1+z\left(r, \alpha_{0}\right)\right)\left(r+\alpha_{0}\left(1+z\left(r, \alpha_{0}\right)\right)\right)}{\left(1+\left(\beta^{2}-2 \beta\right)\left(1-\alpha_{0} z\left(r, \alpha_{0}\right)\right)\right)^{2}\left(r+\alpha_{0}\left(1+z\left(r, \alpha_{0}\right)\right)^{2}\right)} \\
& =\frac{(1-\beta)^{2} z\left(r, \alpha_{0}\right)\left(1+z\left(r, \alpha_{0}\right)\right)^{2}}{\left(1+\left(\beta^{2}-2 \beta\right)\left(1-\alpha_{0} z\left(r, \alpha_{0}\right)\right)\right)^{2}\left(r+\alpha_{0}\left(1+z\left(r, \alpha_{0}\right)\right)^{2}\right)}, \\
\operatorname{SINR}_{i}= & \frac{p_{i} U_{1 i}^{2}}{1+U_{2 i}} \stackrel{p_{i} z\left(r, \alpha_{0}\right)^{2}\left(r+\alpha_{0}\left(1+z\left(r, \alpha_{0}\right)\right)^{2}\right)}{\rightarrow} \frac{\left.\left.p_{0}\right)\left(r, \alpha_{0}\right)\right)^{2}\left(r+\alpha_{0}\left(1+z\left(r, \alpha_{0}\right)\right)^{2}\right)+r z\left(r, \alpha_{0}\right)\left(\frac{1}{K} \sum_{j=1, j \neq i}^{K} p_{j}\right)}{\left(1+z\left(r, \alpha^{2}\right)\right.}
\end{aligned}
$$

This is because given $\alpha_{0} z\left(r, \alpha_{0}\right)<0.5$, when $t=1$, we have $\phi_{2}\left(r, \alpha_{0}\right)=0$, then the $\beta$ set for the case of $t=1$ in (68), i.e., $\phi_{1}\left(r, \alpha_{0}\right)<\beta \leq 1$, or $0 \leq \beta<\phi_{2}\left(r, \alpha_{0}\right)$, reduces to $\phi_{1}\left(r, \alpha_{0}\right)<\beta \leq 1$, which is the same as that in (67) for $t=1$ case.

2.2) If $\alpha_{0} z\left(r, \alpha_{0}\right) \geq 0.5$, then we have $\frac{1}{2\left(1-\alpha_{0} z\left(r, \alpha_{0}\right)\right)} \geq 1$ and $\frac{1}{2\left(1-\alpha_{0} z\left(r, \alpha_{0}\right)\right)} \geq \frac{1}{2} \frac{1}{\sqrt{\alpha_{0} z\left(r, \alpha_{0}\right)\left(1-\alpha_{0} z\left(r, \alpha_{0}\right)\right)}}$. In this case, according to (i) and (ii), we have when $0 \leq t<1$, $\max \left\{0, \phi_{1}\left(r, \alpha_{0}\right)\right\}=\phi_{1}\left(r, \alpha_{0}\right)$, while when $1 \leq t \leq$ $\frac{1}{2} \frac{1}{\sqrt{\alpha_{0} z\left(r, \alpha_{0}\right)\left(1-\alpha_{0} z\left(r, \alpha_{0}\right)\right)}}, \max \left\{0, \phi_{1}\left(r, \alpha_{0}\right)\right\}=0$; it always has $\max \left\{0, \phi_{2}\left(r, \alpha_{0}\right)\right\}=0$. Thus (66) becomes,

$$
\left\{\begin{array}{l}
\phi_{1}\left(r, \alpha_{0}\right)<\beta \leq 1 \\
0<\beta \leq 1, \text { when } 1 \leq t \leq \frac{1}{2} \frac{\text { when } 0 \leq t<1}{\sqrt{\alpha_{0} z\left(r, \alpha_{0}\right)\left(1-\alpha_{0} z\left(r, \alpha_{0}\right)\right)}} .
\end{array}\right.
$$

Therefore, combining the results from 1) and 2), we can obtain the range of $\beta$ that satisfies (19) is given by Proposition 3.

\section{E. Proof for Proposition 4}

Applying the definition of $\rho\left(r, \alpha_{0}, \beta\right)$ into the inequality $\rho\left(r, \alpha_{0}, \beta\right) \geq \frac{Q_{a v}}{P_{a v}}$, we have,

$$
\begin{aligned}
& \left(\beta+\frac{1}{2 t\left(1-\alpha_{0} z\left(r, \alpha_{0}\right)\right)}-1\right)^{2} \\
& \leq 1-\frac{1}{1-\alpha_{0} z\left(r, \alpha_{0}\right)}+\frac{1}{4 t^{2}\left(1-\alpha_{0} z\left(r, \alpha_{0}\right)\right)^{2}}
\end{aligned}
$$

Obviously, the right hand side (RHS) of (70) must be nonnegative, which gives $t \leq \frac{1}{2} \frac{1}{\sqrt{\alpha_{0} z\left(r, \alpha_{0}\right)\left(1-\alpha_{0} z\left(r, \alpha_{0}\right)\right)}}$. Then from (70), we have, $\phi_{2}\left(r, \alpha_{0}\right) \leq \beta \leq \phi_{1}\left(r, \alpha_{0}\right)$. Thus, (22) becomes,

$$
\max \left\{0, \phi_{2}\left(r, \alpha_{0}\right)\right\} \leq \beta \leq \max \left\{0, \phi_{1}\left(r, \alpha_{0}\right)\right\}
$$

Based on the (i) and (ii) in Appendix D, similar to 2.1) and 2.2) in Appendix D, we can obtain,

(1) If $\alpha_{0} z\left(r, \alpha_{0}\right)<0.5$, then (71) becomes,

$$
\left\{\begin{array}{l}
0 \leq \beta \leq \phi_{1}\left(r, \alpha_{0}\right) \quad \text { when } \quad 0 \leq t \leq 1 \\
\phi_{2}\left(r, \alpha_{0}\right) \leq \beta \leq \phi_{1}\left(r, \alpha_{0}\right) \\
\text { when } \quad 1<t \leq \frac{1}{2} \frac{1}{\sqrt{\alpha_{0} z\left(r, \alpha_{0}\right)\left(1-\alpha_{0} z\left(r, \alpha_{0}\right)\right)}}
\end{array}\right.
$$

(72) can be rewritten as,

$$
\left\{\begin{array}{l}
0 \leq \beta \leq \phi_{1}\left(r, \alpha_{0}\right) \quad \text { when } \quad 0 \leq t<1 \\
\phi_{2}\left(r, \alpha_{0}\right) \leq \beta \leq \phi_{1}\left(r, \alpha_{0}\right) \\
\text { when } \quad 1 \leq t \leq \frac{1}{2} \frac{1}{\sqrt{\alpha_{0} z\left(r, \alpha_{0}\right)\left(1-\alpha_{0} z\left(r, \alpha_{0}\right)\right)}}
\end{array}\right.
$$

This is because given $\alpha_{0} z\left(r, \alpha_{0}\right)<0.5$, when $t=1$, we have $\phi_{2}\left(r, \alpha_{0}\right)=0$, then the $\beta$ set for the case of $t=1$ in (73), i.e., $\phi_{2}\left(r, \alpha_{0}\right) \leq \beta \leq \phi_{1}\left(r, \alpha_{0}\right)$, becomes $0 \leq \beta \leq \phi_{1}\left(r, \alpha_{0}\right)$, which is the same as that in (72) for $t=1$ case.

(2) If $\alpha_{0} z\left(r, \alpha_{0}\right) \geq 0.5$, then (71) becomes,

$$
\left\{\begin{array}{l}
0 \leq \beta \leq \phi_{1}\left(r, \alpha_{0}\right) \\
\beta=0, \quad \text { when } \quad 1 \leq t \leq \frac{1}{2} \frac{\text { when } \quad 0 \leq t<1}{\sqrt{\alpha_{0} z\left(r, \alpha_{0}\right)\left(1-\alpha_{0} z\left(r, \alpha_{0}\right)\right)}} .
\end{array}\right.
$$

Need to note that one of constraints in (22), i.e., $\rho\left(r, \alpha_{0}, \beta\right) \geq \frac{Q_{a v}}{P_{a v}}=t^{2}$, gives $t \leq \sqrt{\rho\left(r, \alpha_{0}, \beta\right)}$, then in (74), when $1<t \leq \frac{1}{2} \frac{1}{\sqrt{\alpha_{0} z\left(r, \alpha_{0}\right)\left(1-\alpha_{0} z\left(r, \alpha_{0}\right)\right)}}$, $\beta=0$ leads to $t \leq \sqrt{\rho\left(r, \alpha_{0}, 0\right)=1}$, which contradicts with $1<t \leq \frac{1}{2} \frac{1}{\sqrt{\alpha_{0} z\left(r, \alpha_{0}\right)\left(1-\alpha_{0} z\left(r, \alpha_{0}\right)\right)}}$. Therefore, (74) should be revised as,

$$
\left\{\begin{array}{lll}
0 \leq \beta \leq \phi_{1}\left(r, \alpha_{0}\right) & \text { when } & 0 \leq t<1 ; \\
\beta=0, & \text { when } & t=1 .
\end{array}\right.
$$

Thus, combining the results of (73) and (75), we can obtain the range of $\beta$ that satisfies the constraints in (22) is given by Proposition 4.

\section{F. Proof for Proposition 5}

Let $\beta_{Q}$ denote the optimal solution of Problem (26). Problem (26) is equivalent to the following optimization problem:

$$
\beta_{Q}=\underset{\beta \in \mathcal{A}_{2}}{\arg \min } \rho\left(r, \alpha_{0}, \beta\right)
$$

According to the Fermat's theorem, every local extremum of $\rho\left(r, \alpha_{0}, \beta\right)$ on the set $\beta \in \mathcal{A}$, satisfies $\frac{\partial \rho\left(r, \alpha_{0}, \beta\right)}{\partial \beta}=0$. Note that, $\frac{\partial \rho\left(r, \alpha_{0}, \beta\right)}{\partial \beta}=\frac{2(1-\beta)}{\left(1+\left(\beta^{2}-2 \beta\right)\left(1-\alpha_{0} z\left(r, \alpha_{0}\right)\right)\right)^{3}}(-1+(1-$ $\left.\left.\alpha_{0} z\left(r, \alpha_{0}\right)\right)\left((1-\beta)^{2}+1\right)\right)$. Since $\frac{2(1-\beta)}{\left(1+\left(\beta^{2}-2 \beta\right)\left(1-\alpha_{0} z\left(r, \alpha_{0}\right)\right)\right)^{3}}>$ 0 on the set $\beta \in[0,1)$, there is only one solution for $\frac{\partial \rho\left(r, \alpha_{0}, \beta\right)}{\partial \beta}=0$, denoted by $\beta_{0}$ and given as, $\beta_{0}=1-$ 
$\sqrt{\frac{1}{1-\alpha_{0} z\left(r, \alpha_{0}\right)}-1}$

1) When $\alpha_{0} z\left(r, \alpha_{0}\right) \geq 0.5$,

In this case, we have $\beta_{0} \leq 0$, then $\frac{\partial \rho\left(r, \alpha_{0}, \beta\right)}{\partial \beta} \leq 0$ on the set $[0,1)$, i.e., $\rho\left(r, \alpha_{0}, \beta\right)$ is a nondecreasing on $[0,1)$. According to Proposition 4 , in this case, $\beta$ must satisfy, $\left\{\begin{array}{ll}0 \leq \beta \leq \phi_{1}\left(r, \alpha_{0}\right) & \text { when } 0 \leq t<1 ; \\ \beta=0, & \text { when } t=1 .\end{array}\right.$ Thus, we have

$$
\beta_{Q}= \begin{cases}\phi_{1}\left(r, \alpha_{0}\right) & \text { when } \quad 0 \leq t<1 \\ 0, & \text { when } \quad t=1 .\end{cases}
$$

2) When $\alpha_{0} z\left(r, \alpha_{0}\right)<0.5$,

In this case, we have $\beta_{0}>0$. Then for $0 \leq \beta<\beta_{0}$, we have $\frac{\partial \rho\left(r, \alpha_{0}, \beta\right)}{\partial \beta}>0$; while, for $\beta_{0}<\beta<1$, we have $\frac{\partial \rho\left(r, \alpha_{0}, \beta\right)}{\partial \beta}<0$. Therefore, $\rho\left(r, \alpha_{0}, \beta\right)$ is first increasing on the set $\left[0, \beta_{0}\right]$, and then decreasing on the set $\left(\beta_{0}, 1\right)$, i.e., $\beta_{0}$ is the maximum point for $\rho\left(r, \alpha_{0}, \beta\right)$. According to Proposition 4 , in this case, $\beta$ must satisfy, $\left\{\begin{array}{l}0 \leq \beta \leq \phi_{1}\left(r, \alpha_{0}\right) \quad \text { when } \quad 0 \leq t<1 ; \\ \phi_{2}\left(r, \alpha_{0}\right) \leq \beta \leq \phi_{1}\left(r, \alpha_{0}\right), \\ \text { when } \quad 1 \leq t \leq \frac{1}{2} \frac{1}{\sqrt{\alpha_{0} z\left(r, \alpha_{0}\right)\left(1-\alpha_{0} z\left(r, \alpha_{0}\right)\right)}} .\end{array}\right.$

2.1) when $0 \leq t<1$, after simple algebra manipulations, we can get $\left.\rho\left(r, \alpha_{0}, \beta\right)\right|_{\beta=\phi_{1}\left(r, \alpha_{0}\right)}=t^{2}<1=\left.\rho\left(r, \alpha_{0}, \beta\right)\right|_{\beta=0}$, which results in $\beta_{Q}=\phi_{1}\left(r, \alpha_{0}\right)$.

2.2) when $1 \leq t \leq \frac{1}{2} \frac{1}{\sqrt{\alpha_{0} z\left(r, \alpha_{0}\right)\left(1-\alpha_{0} z\left(r, \alpha_{0}\right)\right)}}$, in this case, $\left.\rho\left(r, \alpha_{0}, \beta\right)\right|_{\beta=\phi_{1}\left(r, \alpha_{0}\right)}=\left.\rho\left(r, \alpha_{0}, \beta\right)\right|_{\beta=\phi_{2}\left(r, \alpha_{0}\right)}=t^{2}$, which gives $\beta_{Q}=\phi_{1}\left(r, \alpha_{0}\right)$ or $\phi_{2}\left(r, \alpha_{0}\right)$.

Combing the results from 2.1) and 2.2), we have

$\beta_{Q}=\left\{\begin{aligned} \phi_{1}\left(r, \alpha_{0}\right) & \text { when } 0 \leq t<1 ; \\ \phi_{1}\left(r, \alpha_{0}\right) & \text { or } \phi_{2}\left(r, \alpha_{0}\right), \\ & \text { when } 1 \leq t \leq \frac{1}{2} \frac{1}{\sqrt{\alpha_{0} z\left(r, \alpha_{0}\right)\left(1-\alpha_{0} z\left(r, \alpha_{0}\right)\right)}} .\end{aligned}\right.$

Therefore, from 1) and 2), we have the optimal solution for Problem (76) is given by Proposition 5.

\section{G. Proof for Proposition 6}

As $P_{a v} \rightarrow \infty, t=\sqrt{\frac{Q_{a v}}{P_{a v}}} \rightarrow 0$. Thus in this case, we have $0 \leq t<1$, and then according to set $\mathcal{A}_{3}$, we have that $\beta^{*}$ must satisfy

$$
\phi_{1}\left(r, \alpha_{0}^{*}\right) \leq \beta^{*} \leq 1
$$

The definition of $\phi_{1}\left(r, \alpha_{0}^{*}\right)$ is given by (21). Note that, as $P_{a v} \rightarrow \infty, \alpha_{0}^{*}=\frac{r}{P_{a v}} \rightarrow 0$, thus we have,

$$
\begin{aligned}
& \lim _{P_{a v} \rightarrow \infty} \alpha_{0}^{*} z\left(r, \alpha_{0}^{*}\right)=\lim _{\alpha_{0}^{*} \rightarrow 0} \alpha_{0}^{*} z\left(r, \alpha_{0}^{*}\right) \\
& =\lim _{\alpha_{0}^{*} \rightarrow 0} \frac{1}{2}\left[\sqrt{(1-r)^{2}+2(1+r) \alpha_{0}^{*}+\left(\alpha_{0}^{*}\right)^{2}}+1-r-\alpha_{0}^{*}\right] \\
& =\frac{1}{2}(|1-r|+1-r)=\left\{\begin{array}{lll}
0, & \text { when } & r \geq 1 \\
1-r, & \text { when } & r<1
\end{array}\right.
\end{aligned}
$$

Applying (80) into the definition of $\phi_{1}\left(r, \alpha_{0}^{*}\right)$ in (21), gives: 1) when $r \geq 1, \lim _{P_{a v} \rightarrow \infty} \phi_{1}\left(r, \alpha_{0}^{*}\right)=1$; 2) when $r<1$, $\lim _{P_{a v} \rightarrow \infty} \phi_{1}\left(r, \alpha_{0}^{*}\right) \stackrel{(a)}{=} \lim _{t \rightarrow 0} \frac{\sqrt{4(r-1) r t^{2}+1}+2 r t-1}{2 r t}=1$, where (a) is due to as $P_{a v} \rightarrow \infty, t=\sqrt{\frac{Q_{a v}}{P_{a v}}} \rightarrow 0$. Based on 1) and 2 ), we can conclude for any given $r, \lim _{P_{a v} \rightarrow \infty} \phi_{1}\left(r, \alpha_{0}^{*}\right)=1$. Therefore, from (79), we can obtain $\lim _{P_{a v} \rightarrow \infty} \beta^{*}=1$.

\section{H. Some useful Lemmas}

Lemma 1: ([38, Corollary 1] obtained directly from [39, Lemma B.26]) Let $\mathbf{A}$ be a deterministic $M \times M$ complex matrix with uniformly bounded spectral norm for all $M$. Let vector $\mathbf{x}=\frac{1}{\sqrt{M}}\left[x_{1}, \ldots, x_{M}\right]^{T}$, which is independent of $\mathbf{A}$, and $x_{i}, \forall i=1, \ldots, M$ are i.i.d complex random variables with zero mean, unit variance and finite eighth order moment. Then as $M \rightarrow \infty, \mathbf{x}^{\mathcal{H}} \mathbf{A x}-\frac{1}{M} \operatorname{tr}\{\mathbf{A}\} \rightarrow 0$ almost surely.

Lemma 2: ([38, Theorem 7] and [22]) Let $\mathbf{x}$ be as defined in Lemma 1 and let $\mathbf{Y}$ be a $M \times K$ matrix of i.i.d. complex random variables, independent of $\mathbf{x}$, with zero mean and variance $\frac{1}{M}$. Assume $r=\lim _{M \rightarrow \infty} \frac{K}{M}$ being held constant, then as $M \rightarrow \infty, \frac{1}{M} \operatorname{tr}\left\{\mathbf{Y} \mathbf{Y}^{\mathcal{H}}+\sigma \mathbf{I}\right\}^{-1} \rightarrow$ $z(r, \sigma)$ almost surely, where $z(r, \sigma)$ is the unique solution of $z(r, \sigma)=\left(\sigma+\frac{r}{1+z(r, \sigma)}\right)^{-1}$ [38, Theorem 7]. In [22], the closed-form solution to above equation was found as $z(r, \sigma)=\frac{1}{2}\left[\sqrt{\frac{(1-r)^{2}}{\sigma^{2}}+\frac{2(1+r)}{\sigma}+1}+\frac{1-r}{\sigma}-1\right]$ and $\frac{\partial z(r, \sigma)}{\partial \sigma}=-\frac{z(r, \sigma)(1+z(r, \sigma))^{2}}{r+\sigma(1+z(r, \sigma))^{2}}$. Thus $\mathbf{x}^{\mathcal{H}}\left(\mathbf{Y} \mathbf{Y}^{\mathcal{H}}+\sigma \mathbf{I}\right)^{-1} \mathbf{x} \rightarrow$ $\frac{1}{M} \operatorname{tr}\left\{\mathbf{Y} \mathbf{Y}^{\mathcal{H}}+\sigma \mathbf{I}\right\}^{-1} \rightarrow z(r, \sigma)$, as $M \rightarrow \infty$.

Remark 14: Note that the original inspiration for this Lemma is the famous Marcenko-Pastur law [42], which can be seen by considering the Stieltjes transformation of the asymptotic eigenvalue distribution of the matrix $\mathbf{Y} \mathbf{Y}^{\mathcal{H}}$.

Lemma 3: ([21, Lemma 6]) Let $\mathbf{A}$ be a deterministic $M \times$ $M$ complex matrix with uniformly bounded spectral norm for all $M$ and $\mathbf{B} \in \mathcal{C}^{M \times M}$ be random Hermitian, with its minimum eigenvalue, denoted as $\lambda_{B}$, satisfying $\exists \epsilon>0, \lambda_{\mathbf{B}}>\epsilon$ for all $M$ with probability one. Then for $\mathbf{x} \in \mathcal{C}^{M}$, as $M \rightarrow \infty$, $\frac{1}{M} \operatorname{tr}\left\{\mathbf{A B} \mathbf{B}^{-1}\right\} \rightarrow \frac{1}{M} \operatorname{tr}\left\{\mathbf{A}\left(\mathbf{B}+\mathbf{x} \mathbf{x}^{\mathcal{H}}\right)^{-1}\right\}$ almost surely, where $\mathbf{B}^{-1}$ and $\left(\mathbf{B}+\mathbf{x} \mathbf{x}^{\mathcal{H}}\right)^{-1}$ exist with probability one as $M \rightarrow \infty$.

Lemma 4: Given bounded $r=\frac{K}{M}$, as $M \rightarrow \infty$, $\frac{1}{M} \operatorname{tr}\left\{\mathbf{C}_{1}^{-1}\right\} \quad \stackrel{a . s .}{\rightarrow} \quad z\left(r, \alpha_{0}\right), \quad \frac{1}{M} \operatorname{tr}\left\{\hat{\mathbf{H}}_{-i} \hat{\mathbf{H}}_{-i}^{\mathcal{H}}\right\} \quad \stackrel{\text { a.s. }}{\rightarrow} r$, $\frac{1}{M} \operatorname{tr}\left\{\mathbf{C}_{1}^{-1} \hat{\mathbf{H}}_{-i} \hat{\mathbf{H}}_{-i}^{\mathcal{H}}\right\} \quad \stackrel{\text { a.s. }}{\rightarrow} \quad 1-\alpha_{0} z\left(r, \alpha_{0}\right) \quad$ and $\frac{1}{M} \operatorname{tr}\left\{\hat{\mathbf{H}}_{-i} \hat{\mathbf{H}}_{-i}^{\mathcal{H}} \mathbf{C}_{1}^{-1} \hat{\mathbf{H}}_{-i} \hat{\mathbf{H}}_{-i}^{\mathcal{H}}\right\} \stackrel{\text { a.s. }}{\rightarrow} \quad r-\alpha_{0}+\alpha_{0}^{2} z\left(r, \alpha_{0}\right)$ where $\mathbf{C}_{1} \triangleq\left(\hat{\mathbf{H}}_{-i} \hat{\mathbf{H}}_{-i}^{\mathcal{H}}+\alpha_{0} \mathbf{I}\right)$ and $z\left(r, \alpha_{0}\right)=$ $\frac{1}{2}\left[\sqrt{\frac{(1-r)^{2}}{\alpha_{0}^{2}}+\frac{2(1+r)}{\alpha_{0}}+1}+\frac{1-r}{\alpha_{0}}-1\right]$.

Proof: Given $\mathbf{C}_{1} \triangleq\left(\hat{\mathbf{H}}_{-i} \hat{\mathbf{H}}_{-i}^{\mathcal{H}}+\alpha_{0} \mathbf{I}\right)$, according to Lemma 3 and Lemma 2 (see Appendix H), with fixed $r=\lim _{M \rightarrow \infty} \frac{K}{M}$, we have, as $M \rightarrow \infty, \frac{1}{M} \operatorname{tr}\left\{\mathbf{C}_{1}^{-1}\right\} \stackrel{\text { a.s. }}{\rightarrow}$ $\frac{1}{M} \operatorname{tr}\left\{\left(\hat{\mathbf{H}} \hat{\mathbf{H}}^{\mathcal{H}}+\alpha_{0} \mathbf{I}\right)^{-1}\right\} \stackrel{a . s .}{\rightarrow} z\left(r, \alpha_{0}\right)$. Following the result in [40, Equation (2.102)] (i.e., $\lim _{M \rightarrow \infty} \frac{1}{M} \operatorname{tr}\left\{\left(\mathbf{Y} \mathbf{Y}^{\mathcal{H}}\right)^{m}\right\}=$ $\sum_{i=1}^{m} \frac{1}{m}\left(\begin{array}{c}m \\ i\end{array}\right)\left(\begin{array}{c}m \\ i-1\end{array}\right) r^{i}$ where $\mathbf{Y}$ is a $M \times K$ matrix of i.i.d. complex random variables with zero mean and variance $\frac{1}{M}$ ) and Lemma 1 , we have, as $M \rightarrow \infty$,

$$
\begin{aligned}
\frac{1}{M} \operatorname{tr}\left\{\hat{\mathbf{H}}_{-i} \hat{\mathbf{H}}_{-i}^{\mathcal{H}}\right\} & =\frac{1}{M} \operatorname{tr}\left\{\hat{\mathbf{H}} \hat{\mathbf{H}}^{\mathcal{H}}\right\}-\frac{1}{M} \hat{\mathbf{h}}_{i}^{\mathcal{H}} \hat{\mathbf{h}}_{i} \\
& \stackrel{\text { a.s. }}{\rightarrow} r-\frac{1}{M^{2}} \operatorname{tr}\{\mathbf{I}\} \stackrel{\text { a.s. }}{\rightarrow} r
\end{aligned}
$$


Then, as $M \rightarrow \infty$, we obtain almost surely,

$$
\begin{aligned}
\frac{1}{M} \operatorname{tr}\left\{\mathbf{C}_{1}^{-1} \hat{\mathbf{H}}_{-i} \hat{\mathbf{H}}_{-i}^{\mathcal{H}}\right\} & =\frac{1}{M} \operatorname{tr}\left\{\mathbf{C}_{1}^{-1}\left(\mathbf{C}_{1}-\alpha_{0} \mathbf{I}\right)\right\} \\
& =1-\alpha_{0} \frac{1}{M} \operatorname{tr}\left\{\mathbf{C}_{1}^{-1}\right\} \stackrel{\text { a.s. }}{\rightarrow} 1-\alpha_{0} z\left(r, \alpha_{0}\right) .
\end{aligned}
$$

Similarly, we have, as $M \rightarrow \infty$,

$$
\begin{aligned}
& \frac{1}{M} \operatorname{tr}\left\{\hat{\mathbf{H}}_{-i} \hat{\mathbf{H}}_{-i}^{\mathcal{H}} \mathbf{C}_{1}^{-1} \hat{\mathbf{H}}_{-i} \hat{\mathbf{H}}_{-i}^{\mathcal{H}}\right\} \\
& =\frac{1}{M} \operatorname{tr}\left\{\hat{\mathbf{H}}_{-i} \hat{\mathbf{H}}_{-i}^{\mathcal{H}}\right\}-\alpha_{0} \frac{1}{M} \operatorname{tr}\left\{\mathbf{C}_{1}^{-1} \hat{\mathbf{H}}_{-i} \hat{\mathbf{H}}_{-i}^{\mathcal{H}}\right\} \\
& \stackrel{\text { a.s. }}{\rightarrow} r-\alpha_{0}+\alpha_{0}^{2} z\left(r, \alpha_{0}\right) .
\end{aligned}
$$

\section{REFERENCES}

[1] A. Goldsmith, S. A. Jafar, I. Maric, and S. Srinivasa, "Breaking spectrum gridlock with cognitive radios: an information theoretic perspective," Proc. IEEE, vol. 97, no. 5, pp. 894-914, May 2009.

[2] R. Zhang and Y. Liang, "Exploiting multi-antennas for opportunistic spectrum sharing in cognitive radio networks," IEEE J. Sel. Topics Signal Process., vol. 2, no. 1, pp. 88-102, Feb. 2008.

[3] L. Zhang, Y.-C. Liang, and Y. Xin, "Joint beamforming and power allocation for multiple access channels in cognitive radio networks," IEEE J. Sel. Areas Commun., vol. 26, no. 1, pp. 38-51, Jan. 2008.

[4] L. Zhang, Y. Xin, and Y. Liang , "Weighted sum rate optimization for cognitive radio MIMO broadcast channels," IEEE Trans. Wireless Commun., vol. 8, no. 6, pp. 2950-2959, June 2009.

[5] G. Caire and S. Shamai, "On the achievable throughput of a multiantenna Gaussian broadcast channel," IEEE Trans. Inf. Theory, vol. 49, no. 7, pp. 1691-1706, July 2003.

[6] A. Tajer, N. Prasad, and X. Wang, "Beamforming and rate allocation in MISO cognitive radio networks," IEEE Trans. Signal Process., vol. 58, no. 1, pp. 362-377, Jan. 2010.

[7] L. Gallo, F. Negro, I. Ghauri, and D. T. M. Slock, "Weighted sum rate maximization in the underlay cognitive MISO interference channel," in Proc. 2011 IEEE PIMRC, pp. 661-665.

[8] S. Kim and G. B. Giannakis, "Optimal resource allocation for MIMO ad hoc cognitive radio networks," IEEE Trans. Inf. Theory, vol. 57, no. 5, pp. 3117-3131, May 2011

[9] K. Jitvanichphaibool, Y. C. Liang, and R. Zhang, "Beamforming and power control for multi-antenna cognitive two-way relaying," in Proc. 2009 IEEE Wireless Commun.and Networking Conf., pp. 1-6.

[10] R. Zhang, Y. Liang, and S. Cui, "Dynamic resource allocation in cognitive radio networks," IEEE Signal Process. Mag., vol. 27, no. 3, pp. 102-114, May 2010.

[11] S. K. Joshi, P. C. Weeraddana, M. Codreanu, and M. Latva-aho, "Weighted sum-rate maximization for MISO downlink cellular networks via branch and bound," IEEE Trans. Signal Process., vol. 60, no. 4, pp. 2090-2095, Apr. 2012.

[12] M. Rossi, A. M. Tulino, O. Simeone, and A. M. Haimovich, "Nonconvex utility maximization in Gaussian MISO broadcast and interference channels," in Proc. 2011 IEEE ICASSP, pp. 2960-2963.

[13] Y. Xu, T. Le-Ngoc, and S. Panigrahi, "Global concave minimization for optimal spectrum balancing in multi-user DSL networks," IEEE Trans. Signal Process., vol. 56, no. 7, pp. 2875-2885, July 2008.

[14] Q. Zhang, C. He, and L. Jiang, "Achieving maximum weighted sumrate in multicell downlink MISO systems," IEEE Commun. Lett., vol. 16, no. 11, pp. 1808-1811, Nov. 2012.

[15] M. Kobayashi and G. Caire, "Joint beamforming and scheduling for a multi-antenna downlink with imperfect transmitter channel knowledge," IEEE J. Sel. Areas Commun., vol. 25, no. 7, pp. 1468-1477, Sept. 2007.

[16] L. Tran, M. F. Hanif, A. Tolli, and M. Juntti, "Fast converging algorithm for weighted sum rate maximization in multicell MISO downlink," IEEE Signal Process. Lett., vol. 19, no. 12, pp. 872-875, Dec. 2012.

[17] Y. Y. He and S. Dey, "Sum rate maximization for cognitive MISO broadcast channels: beamforming design and large systems analysis." Available: https://sites.google.com/site/zhyfutopia/Home/Longversion.pdf

[18] X. Kang, Y. Liang, A. Nallanathan, and H. K. Garg, "Optimal power allocation for fading channels in cognitive radio networks: ergodic capacity and outage capacity," IEEE Trans. Wireless Commun., vol. 8, no. 2, pp. 940-950, Feb. 2009.
[19] Y. Y. He and S. Dey, "Power allocation in spectrum sharing cognitive radio networks with quantized channel information," IEEE Trans. Commun., vol. 59, no. 6, pp. 1644-1656, June 2011.

[20] C. B. Peel, B. M. Hochwald, and A. L. Swindlehurst, "A vector-perturbation technique for near-capacity multiantenna multiuser communication-part I: channel inversion and regularization," IEEE Trans. Commun., vol. 53, no. 1, pp. 195-202, Jan. 2005.

[21] S. Wagner, R. Couillet, M. Debbah, and D. T. M. Slock, "Large system analysis of linear precoding in correlated MISO broadcast channels under limited feedback," IEEE Trans. Inf. Theory, vol. 58, no. 7, pp. 4509-4537, July 2012.

[22] V. K. Nguyen, R. Muharar, and J. Evans, "Multiuser transmit beamforming via regularized channel inversion: a large system analysis," Available: http://www.cubinlab.ee.unimelb.edu.au/ rmuharar/doc/manuscriptrevRusd ha22-11.pdf

[23] R. Muharar and J. Evans, "Downlink beamforming with transmit-side channel correlation: a large system analysis," in Proc. 2011 IEEE Int. Conference on Commun., pp. 1-5.

[24] E. Björnson, J. Hoydis, M. Kountouris, and M. Debbah, "Massive MIMO systems with non-ideal hardware: energy efficiency, estimation, and capacity limits," preprint, 2013. Available: http://arxiv.org/abs/1307.2584.

[25] R. Muharar, R. Zakhour, and J. Evans, "Optimal power allocation and user loading for multiuser MISO channels with regularized channel inversion," preprint, 2013. Available: http://arxiv.org/pdf/1307.1537v2.pdf

[26] J. Zhang, C.-K. Wen, S. Jin, X. Q. Gao, and K.-K. Wong, "Large system analysis of cooperative multi-cell downlink transmission via regularized channel inversion with imperfect CSIT," IEEE Trans. Wireless Commun., vol. 12 , no. 10 , pp. 4801-4813, Oct. 2013.

[27] Z. D. Bai and J. W. Silverstein, "No eigenvalues outside the support of the limiting spectral distribution of large dimensional sample covariance matrices," Ann. Probab., vol. 26, no. 1, pp. 316-345, Jan. 1998.

[28] Y. Y. He and S. Dey, "Weighted sum rate maximization for cognitive MISO broadcast channel: large system analysis," in Proc. 2013 ICASSP, 4 pages.

[29] A. Ghasemi and E. S. Sousa, "Fundamental limits of spectrum-sharing in fading environments," IEEE Trans. Wireless Commun., vol. 6, no. 2, pp. 649-658, Feb. 2007.

[30] R. Zhang, S. Cui, and Y.-C. Liang, "On ergodic sum capacity of fading cognitive multiple-access and broadcast channels," IEEE Trans. Inf. Theory, vol. 55, no. 11, pp. 5161-5178, Nov. 2009.

[31] R. Zhang and S. Cui "Cooperative interference management with MISO beamforming," IEEE Trans. Signal Process., vol. 58, no. 10, pp. 54505458, Oct. 2010.

[32] J. Papandriopoulos and J. S. Evans, "SCALE: a low-complexity distributed protocol for spectrum balancing in multiuser DSL networks," IEEE Trans. Inf. Theory, vol. 55, no. 8, pp. 3711-3724, Aug. 2009.

[33] K. Lee and I. Lee, "MMSE based block diagonalization for cognitive radio MIMO broadcast channels," IEEE Trans. Wireless Commun., vol. 10, no. 10 , pp. $3139-3144$, Oct. 2011

[34] L. Zhang, Y.-C. Liang, Y. Pei, and R. Zhang, "Robust beamforming design: from cognitive radio MISO channels to secrecy MISO channels," in Proc. 2009 IEEE GLOBECOM, pp. 1-5.

[35] S. Wagner and D. T. M. Slock, "Weighted sum rate maximization of correlated MISO broadcast channels under linear precoding: a large system analysis," in Proc. 2011 IEEE Signal Process. Advances in Wireless Commun., pp. 411-415.

[36] G. Geraci, J. Yuan, and I. B. Collings, "Large system analysis of the secrecy sum-rates with regularized channel inversion precoding," in Proc. 2012 IEEE Wireless Communications and Networking Conference, pp. 533-537.

[37] W. Liao, T. Chang, W. Ma, and C. Chi, "QoS-based transmit beamforming in the presence of eavesdroppers: an optimized artificial-noise-aided approach," IEEE Trans. Signal Process., vol. 59, no. 3, pp. 1202-1216, Mar. 2011.

[38] J. Evans and D. N. C. Tse, "Large system performance of linear multiuser receivers in multipath fading channels," IEEE Trans. Inf. Theory, vol. 46, no. 6, pp. 2059-2078, Sept. 2000.

[39] Z. D. Bai and J. W. Silverstein, Spectral Analysis of Large Dimensional Random Matrices, 2nd ed. Springer, 2010.

[40] A. M. Tulino and S. Verdú, "Random matrix theory and wireless communications," Foundations Trends Commun. Inf. Theory, vol. 1, no. 1, pp. 1-182, 2004.

[41] W. Hachem et al., "Deterministic equivalents of certain functionals of large random matrices," The Annals of Applied Probability, vol. 17, no. 3, pp. 875-930, 2007.

[42] V. A. Marčenko and L. A. Pastur, "Distribution of eigenvalues for some sets of random matrices." Sbornik: Mathematics 1.4 (1967), pp. 457-483. 


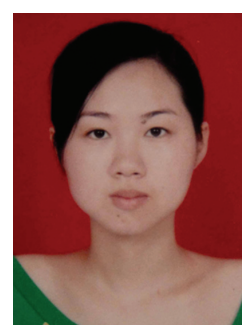

Yuanyuan He (S'08-M'12) received her B.Eng. degree in communication engineering from Yanshan University, China, in 2005, the M.Eng. degree in electronic engineering from Harbin Institute of Technology, China, in 2007, and the Ph.D. degree in the Department of Electrical and Electronic Engineering, University of Melbourne, Melbourne, Australia, in 2011, where she was a Postdoctoral Research Fellow from 2011 to April 2013. She is currently a Postdoctoral Research Fellow in Monash University, Melbourne, Australia, since June 2013. Her research interests lie in the area of wireless communications including resource allocation, cognitive radio, physical-layer security and limited feedback.

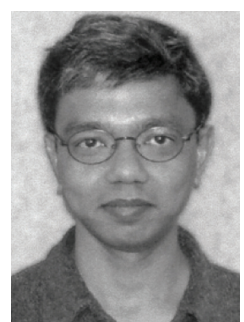

Subhrakanti Dey (SM'06) was born in India, in 1968. He received the B.Tech. and M.Tech. degrees from the Department of Electronics and Electrical Communication Engineering, Indian Institute of Technology, Kharagpur, India, in 1991 and 1993, respectively, and the Ph.D. degree from the Department of Systems Engineering, Research School of Information Sciences and Engineering, Australian National University, Canberra, Australia, in 1996.

Since June 2013, he is a Professor in the Division of Signals and Systems, Dept. of Eng. Sciences, Uppsala University in Sweden. Prior to this, he was with the Department of Electrical and Electronic Engineering, University of Melbourne, Parkville, Australia, since February 2000, where he was a full Professor since 2007. From September 1995 to September 1997 and September 1998 to February 2000, he was a postdoctoral Research Fellow with the Department of Systems Engineering, Australian National University. From September 1997 to September 1998, he was a post-doctoral Research Associate with the Institute for Systems Research, University of Maryland, College Park. His current research interests include networked control systems, wireless communications and networks, signal processing for sensor networks, and stochastic and adaptive estimation and control. Prof. Dey currently serves on the Editorial Board of Elsevier Systems and Control Letters. He was also an Associate Editor for the IEEE TRANSACTIONS ON SIGNAL PROCESSING and the IEEE Transactions on Automatic Control. He is a Senior Member of IEEE. 